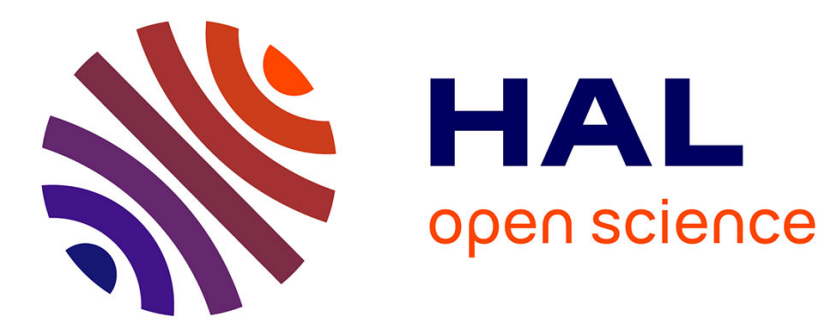

\title{
ON THE SELF-DISPLACEMENT OF DEFORMABLE BODIES IN A POTENTIAL FLUID FLOW
}

Alexandre Munnier

\section{To cite this version:}

Alexandre Munnier. ON THE SELF-DISPLACEMENT OF DEFORMABLE BODIES IN A POTENTIAL FLUID FLOW. Mathematical Models and Methods in Applied Sciences, 2008, 18 (11), pp.1945-1981. 10.1142/S021820250800325X . inria-00589796

\section{HAL Id: inria-00589796 \\ https://hal.inria.fr/inria-00589796}

Submitted on 2 May 2011

HAL is a multi-disciplinary open access archive for the deposit and dissemination of scientific research documents, whether they are published or not. The documents may come from teaching and research institutions in France or abroad, or from public or private research centers.
L'archive ouverte pluridisciplinaire HAL, est destinée au dépôt et à la diffusion de documents scientifiques de niveau recherche, publiés ou non, émanant des établissements d'enseignement et de recherche français ou étrangers, des laboratoires publics ou privés. 


\title{
ON THE SELF-DISPLACEMENT OF DEFORMABLE BODIES IN A POTENTIAL FLUID FLOW
}

\author{
ALEXANDRE MUNNIER
}

\begin{abstract}
Understanding fish-like locomotion as a result of internal shape changes may result in improved underwater propulsion mechanism. In this article, we study a coupled system of partial differential equations and ordinary differential equations which models the motion of self-propelled deformable bodies (called swimmers) in an potential fluid flow. The deformations being prescribed, we apply the least action principle of Lagrangian mechanics to determine the equations of the inferred motion. We prove that the swimmers degrees of freedom solve a second order system of nonlinear ordinary differential equations. Under suitable smoothness assumptions on the fluid's domain boundary and on the given deformations, we prove the existence and regularity of the bodies rigid motions, up to a collision between two swimmers or between a swimmer with the boundary of the fluid. Then we compute explicitly the Euler-Lagrange equations in terms of the geometric data of the bodies and of the value of the fluid's harmonic potential on the boundary of the fluid.
\end{abstract}

\section{INTRODUCTION}

1.1. History. Initiated by the mathematician, physician and physiologist G. A. Borelli[4] in 1680 and 1681 and continued much later by zoologists and engineers like C. M. Breder[6] and J. Gray[16],[17] in a series of papers, the investigations to understand the locomotion of aquatic animals have given rise, to numerous scientific publications. This constant interest has grown from the observation that aquatic mammals and fish evolved swimming capabilities far superior to what has been achieved by naval technology. A better understanding of the biomechanics of swimming may allow one to improve the efficiency, manoeuvrability and stealth of underwater vehicles.

During the first half of the 20th century, research was done largely by zoologists and engineers. In the early 1950's the mathematically oriented scientists entered the scene and began to develop theories by which it became possible to make qualitative analysis of swimming propulsion as a continuation of the previously developed quantitative theories. For example, Sir. G. Taylor studies both large and microscopic swimming animals in Ref. [36], [35]. Significant contributions are due to T. Y. Wu[39], J. Lighthill[26] and S. Childress[10]. Recently, J. A. Sparenberg published a survey on the mathematical theory of fish locomotion[34] to which we refer for a more complete bibliography on this period.

In the past few years, serious efforts have emerged to study, build and control underwater vehicles that move and steer by changes of shape and not by direct

2000 Mathematics Subject Classification. AMS Subject Classification: 74F10, 70S05, 76B03, 49Q10.

Key words and phrases. Underwater locomotion; potential fluid flow; Lagrangian mechanics; PDE-ODE coupled system; shape sensitivity analysis. 
propulsion, such as propellers; see for example the works of M. and G. Triantafyllou and D. K. P. Yue[37], S. D. Kelly and R. M. Murray[23] and E. Kanso, J. E. Marsden, C. W. Rowley and J. B. Melli-Huber[21] and references therein.

Experiments have shown that the vortices generated by the tail of fishes play a crucial role to understand their locomotion. Some models incorporate artificially vortices, like those of R. Mason[28] and Q. Zhu, M. J. Wolfgang, D. K. P. Yue and M. S. Triantafyllou[40], detailed in Ref. [15], [22] and [24]. If we do not neglect the viscosity effects, the relevant model consists in the non stationary Navier-Stokes equations for the fluid coupled with Newton's laws for the fish-like swimming object. This perspective is adopted by J. Carling, T. Williams and G. Bowtell[7], H. Liu and K. Kawachi[27] and J. San Martin, J. F. Sheid, T. Takahashi and M. Tucsnak[32]. Let us mention also the pioneering contribution to this research area of G. P. Galdi. He investigates, in the case of a fluid-rigid system filling the whole space, the existence of self-propelled motion in Ref. [14], [13].

Nevertheless, among numerous mathematical articles studying fish locomotion, most of them address the case of a potential flow which is by definition vortex-free. It is the point of view we have chosen in what follows and on which we focus from now on.

1.2. Swimming in a potential flow. The study the of the coupled dynamics of a solid immersed in an infinite extended ideal fluid was initiated by Thomson, Tait, and Kirchhoff. Sir H. Lamb explains their approach in Ref. [25, chap. VI, page 160]: The cardinal feature of the methods followed by these writers consists in this, that the solids and the fluid are treated as forming together one dynamical system, and thus the troublesome calculation of the effect of the fluid pressures on the surfaces of the solids is avoided. They introduce as unknowns of the problem a moment-like quantity called impulse. Denoting respectively $\mathbf{F}$ and $\mathbf{T}$ the extraneous force and torque acting on the solid and applying to the system fluid-solid the principle of conservation of linear and angular momenta, they derive the equations of motion which take the form:

$$
\dot{\Pi}=\Pi \times \boldsymbol{\omega}+\mathbf{P} \times \mathbf{v}+\mathbf{T}, \quad \dot{\mathbf{P}}=\mathbf{P} \times \boldsymbol{\omega}+\mathbf{F} .
$$

In the above system, $(\boldsymbol{\Pi}, \mathbf{P})^{T}$ is the impulse and $\boldsymbol{\omega}, \mathbf{v}$ are respectively the angular and translational velocity vectors of the body relative to a body fixed frame. The first component of the impulse is the body angular momenta, $\boldsymbol{\Pi}:=\left(\left[I_{a c}\right]+\left[I_{a d}\right]\right) \boldsymbol{\omega}$ where $\left[I_{a c}\right]$ is the actual inertia matrix of the solid and $\left[I_{a d}\right]$ is the so-called added inertia matrix. The second component is the body's linear momentum $\mathbf{P}:=\left(m\left[\mathbb{I}_{3}\right]+\right.$ $\left.\left[M_{a d}\right]\right) \mathbf{v}$, in which $m$ is the actual mass of the solid, $\left[\mathbb{I}_{3}\right]$ the $3 \times 3$ identity matrix and $\left[M_{a d}\right]$ the added mass matrix. Both added inertia matrix and added mass matrix depend on the density of the fluid and on the geometry of the fluid's domain, through the solution of a boundary value problem. In the case of a solid alone in an boundless fluid, $\left[I_{a d}\right]$ and $\left[M_{a d}\right]$ are both constant, since the fluid's domain as viewed by an observer attached to the solid remains unchanged in the motion.

Later in his book[25, chap IV, page 187], Sir H. Lamb tackles a more involved problem: When we have more than one moving solid, or when the fluid is bounded, wholly or in part, by fixed walls, we may have recourse to Lagrange's method of 'generalized co-ordinates'. Indeed, introducing the generalized coordinates $\mathbf{q}:=$ $\left(q_{1}, \ldots, q_{n}\right)^{\top}$ of the problem and the generalized velocities $\dot{\mathbf{q}}:=\left(\dot{q}_{1}, \ldots, \dot{q}_{n}\right)^{\top}$, they show that the kinetic energy of the system fluid-solids reads $\mathcal{K}=\dot{\mathbf{q}} \cdot[K(\mathbf{q})] \dot{\mathbf{q}} / 2$ 
where the $n \times n$ matrix $[K(\mathbf{q})]$ is the virtual mass matrix. Its entries $K_{i j}(\mathbf{q})$ depend on the mass and on the inertia matrices of the solids but also on the density of the fluid and on the geometry of the fluid's domain through the solution of a boundary value problem. Contrary to what happens in the case of a single solid, when there are several solids or fixed walls, the virtual mass matrix is no longer constant but does depend on the relative position of the immersed objects. The least action principle of Lagrangian's mechanics yields the equations of motion:

$$
\frac{d}{d t} \partial_{\dot{q}_{i}} \mathcal{K}-\partial_{q_{i}} \mathcal{K}=Q_{i}, \quad i=1, \ldots, n,
$$

where $Q_{i}$ are generalized components of force. Plugging the expression of $\mathcal{K}$ into (1.2), they obtain a system of ODE's for $q_{1}, \ldots, q_{n}$ :

$$
\frac{d}{d t}\left(\sum_{j} K_{i j}(\mathbf{q}) \dot{q}_{j}\right)-\frac{1}{2} \sum_{j, k} \partial_{q_{i}} K_{j k}(\mathbf{q}) \dot{q}_{j} \dot{q}_{k}=Q_{i}, \quad i=1, \ldots, n .
$$

Notice however that before writing this equation, we have to prove that the mappings $\mathbf{q} \mapsto M_{i j}(\mathbf{q})$ is smooth enough to be differentiated. Since the expression of $[K(\mathbf{q})]$ involves the solution of a boundary value problem on the fluid's domain, we must study the sensitivity of a solution of a PDE with respect to the geometry of its domain. The regularity of the boundaries of the solids and fluid will play a crucial role when discussing this point. After proving that $\partial_{q_{i}} K_{i j}$ is well defined, the next step would be to compute explicitly its expression. Invoking the chain rule, we can rewrite the ODE's (1.3):

$$
\sum_{j} K_{i j}(\mathbf{q}) \ddot{q}_{j}+\sum_{j, k} \partial_{q_{k}} K_{i j}(\mathbf{q}) \dot{q}_{k} \dot{q}_{j}-\frac{1}{2} \sum_{j, k} \partial_{q_{i}} K_{j k}(\mathbf{q}) \dot{q}_{j} \dot{q}_{k}=Q_{i}, \quad i=1, \ldots, n .
$$

Although these equations have been studied in the cases of simple geometries (for example, when the solids are a set of spheres or of ellipsoids) by performing explicit computations, they have not, to our knowledge, been tackled in the general case, at least not until very recently. In Ref. [12] we prove, assuming only that the boundaries of solids and the fluid are Lipschitz continuous (which allows corners), that the mass matrix is infinitely differentiable with respect to the generalized coordinates and that the system of ODE's (1.4) is well posed and admits a unique solution which is also infinitely differentiable. As a corollary of a more general result presented later in in this paper, we will slightly improve this result and prove that both the mass matrix and the solutions of (1.4) are in fact analytic.

An alternative approach, when several immersed objects are involved, is to assume that they are hydrodynamically decoupled. It means that the added inertia and added mass associated with a given body is not affected by the presence of the others. Since each body is considered as being alone in the fluid, the impulse method applies.

Settling the equations of motion for shape-changing bodies is a more involved task. When the deformable body is an articulated system of solids connected via ball-and-socket or hinge joints, E. Kanso, J. E. Marsden, C. W. Rowley and J. B. Melli-Huber[21] prove that the impulse method can be adapted, assuming the fluid to be of infinite extend. They obtain equations quite similar to (1.1). The proof is based on the observation that the Lagrangian of the system is invariant under superimposed rigid motions. The limitations of this approach are that no 
extraneous force like the buoyant force can be taken into account, the fluid has to be free of any other object and the motion has to start from rest. Some authors assume in addition that each link is hydrodynamically decoupled from the others. This hypothesis leads to significative simplifications. However, E. Kanso, J. E. Marsden, C. W. Rowley and J. B. Melli-Huber[21] and J. B. Melli, C. W. Rowley and D. S. Rufat[29] show with numerical simulations that in this case the motion differs from that of the same system in its full complexity.

In this paper we study the locomotion of a set of shape-changing bodies in a fluid that can be partially or totally bounded. We derive the equations of motions from Lagrangian mechanics and obtain a system of ODE's roughly similar to (1.4) for the generalized coordinates (which are the degrees of freedom of the bodies). We prove the existence, uniqueness and regularity of solutions assuming that the boundaries of the bodies and the fluid are Lipschitz continuous only. The key point consists in studying the regularity of the potential of the flow with respect to the geometry of the fluid's domain. This task is achieved using tools of shape optimization theory. Similar techniques are being developed in the area of fluid-structures dynamics by M. Moubachir and J. P. Zolesio[30].

Our second contribution is to write the system of ODE's in a form that is convenient for numerical simulation and for studying locomotion. The equations involve, among other quantities, geometric data of the bodies, like the curvature of their boundaries.

1.3. Outline of the paper. This paper is organized as follows: after determining the equations of motion and stating the main results in the next section, we compute the Lagrangian of the system bodies-fluid in Section 3. In Section 4, we address an abstract shape optimization problem whose results are used in Section 5 in the proofs of Theorem 1 and Corollary 1. Section 6 is devoted to the proof of Theorem 2 and in Section 7 we indicate some future directions of investigations. The Appendices contain technical results.

1.4. Notation. Throughout this article, we shall use bold print notations for vectors like $\mathbf{x}, \mathbf{h}, \mathbf{q}$ whereas we keep the usual characters for their coordinates $x_{j}, h_{j}$, $q_{j}$ and in a general way for any real valued functions like $\phi, \varphi$. The canonical basis of the physical space $\mathbb{R}^{3}$ is $\left\{\mathbf{e}_{1}, \mathbf{e}_{2}, \mathbf{e}_{3}\right\}$.

A matrix is denoted in square brackets $[M]$, its entries are $M_{j_{1} j_{2}},[M]^{T}$ is the transposed matrix, $\left[\mathbb{I}_{3}\right]$ is the identity matrix of $\mathbb{R}^{3}$ and $\left[\mathbb{O}_{3}\right]$ the null matrix. The Jacobian matrix of a differentiable function $\varphi$ is $[D \varphi]$.

The Euclidian norm in $\mathbb{R}^{3}$ is denoted $|\mathbf{x}|$ and we keep the same notation for the associated matrix norm $|[M]|:=\sup _{|\mathbf{x}|=1}|[M] \mathbf{x}|,[M] \in \mathcal{M}(3)$ (the vector space of the $3 \times 3$ matrices).

We use double square brackets for third-rank tensors like $\llbracket T \rrbracket$. Its entries are $T_{j_{1} j_{2} j_{3}}$. We use the convention $(\llbracket T \rrbracket \mathbf{q})_{j_{1} j_{2}}:=\sum_{j_{3}} T_{j_{1} j_{2} j_{3}} q_{j_{3}}$ and $(\langle\llbracket T \rrbracket, \mathbf{p}, \mathbf{q}\rangle)_{j_{1}}:=$ $\sum_{j_{2}} \sum_{j_{3}} T_{j_{1} j_{2} j_{3}} p_{j_{2}} q_{j_{3}}$, where $q_{i}$ and $p_{i}$ are the coordinates of $\mathbf{q}$ and $\mathbf{p}$.

If $E_{k}, k=1, \ldots, p$ and $F$ are $p+1$ Banach spaces, we denote $L_{p}\left(E_{1} \times \ldots \times E_{p}, F\right)$ the Banach space of the $p$-linear continuous maps from $E_{1} \times \ldots \times E_{p}$ into $F$.

\section{Modelling AND MAin Results}

2.1. Modelling. We denote by $S(t):=\cup_{i=1}^{n} S^{i}(t)$ the region of the $n$ swimmers at time $t$ and by $\Omega(t)$ the domain of the surrounding fluid. The boundary $\partial \Omega(t)$ 
of the fluid is decomposed into the boundary shared with the swimmers $\Gamma_{2}(t):=$ $\cup_{i=1}^{n} \Gamma_{2}^{i}(t):=\cup_{i=1}^{n} \partial S^{i}(t)$ and $\Gamma_{1}:=\partial \Omega(t) \backslash \Gamma_{2}(t)$. We set $\Omega:=\Omega(0), S_{0}^{i}:=S^{i}(0)$, $S_{0}:=S(0)$ and $\Gamma_{2}:=\Gamma_{2}(0)$. We will assume in the sequel that $\Omega$ is an open and connected set, that $\Gamma_{2}$ is compact and that $\Gamma_{1}$ is either bounded, either an hyperplane. Let $\mathcal{F}$ be an inertial reference frame. In $\mathcal{F}$, the coordinates of the center of mass of the $i$-th swimmer are $\mathbf{h}^{i}(t):=\left(h_{1}^{i}(t), h_{2}^{i}(t), h_{3}^{i}(t)\right)^{T}$ at time $t$. We fix a frame $\mathcal{F}^{i}(t)$ of origin $\mathbf{h}^{i}(t)$ to each swimmer and we write $\mathbf{h}_{0}^{i}:=\mathbf{h}^{i}(0)$ and $\mathcal{F}^{i}(0):=\mathcal{F}_{0}^{i}$. Considering $M^{i}(t)$ a material point of $S^{i}(t)$ of coordinates $\mathbf{x}^{i}(t):=$ $\left(x_{1}^{i}(t), x_{2}^{i}(t), x_{3}^{i}(t)\right)^{T}$ in $\mathcal{F}$ and $\mathbf{y}^{i}(t):=\left(y_{1}^{i}(t), y_{2}^{i}(t), y_{3}^{i}(t)\right)^{T}$ in $\mathcal{F}^{i}(t)$, there exists a rotation matrix $\left[R^{i}(t)\right] \in S O(3)$ (the rotation group of $\mathbb{R}^{3}$ ) such that $\mathbf{x}^{i}(t)=$ $\left[R^{i}(t)\right] \mathbf{y}^{i}(t)+\mathbf{h}^{i}(t)$. We can assume, without loss of generality, that $\left[R^{i}(0)\right]=\left[\mathbb{I}_{3}\right]$. We assume that the motion of $M^{i}(t)$ in $\mathcal{F}^{i}(t)$ is only due to the deformation of the body $S^{i}(t)$. Introducing then $S_{d}^{i}(t):=\left[R^{i}(t)\right]^{T}\left(S^{i}(t)-\mathbf{h}^{i}(t)\right)$, the deformation of the body from its initial states $S_{0}^{i}$ into $S_{d}^{i}(t)$ is described in $\mathcal{F}_{0}^{i}$ by a given oneto-one smooth function $\boldsymbol{\Phi}^{i}\left(t, \mathbf{y}_{0}^{i}\right)$ where $\mathbf{y}_{0}^{i}:=\mathbf{y}^{i}(0)=\mathbf{x}_{0}^{i}-\mathbf{h}_{0}^{i}$ (see Figure 1). We

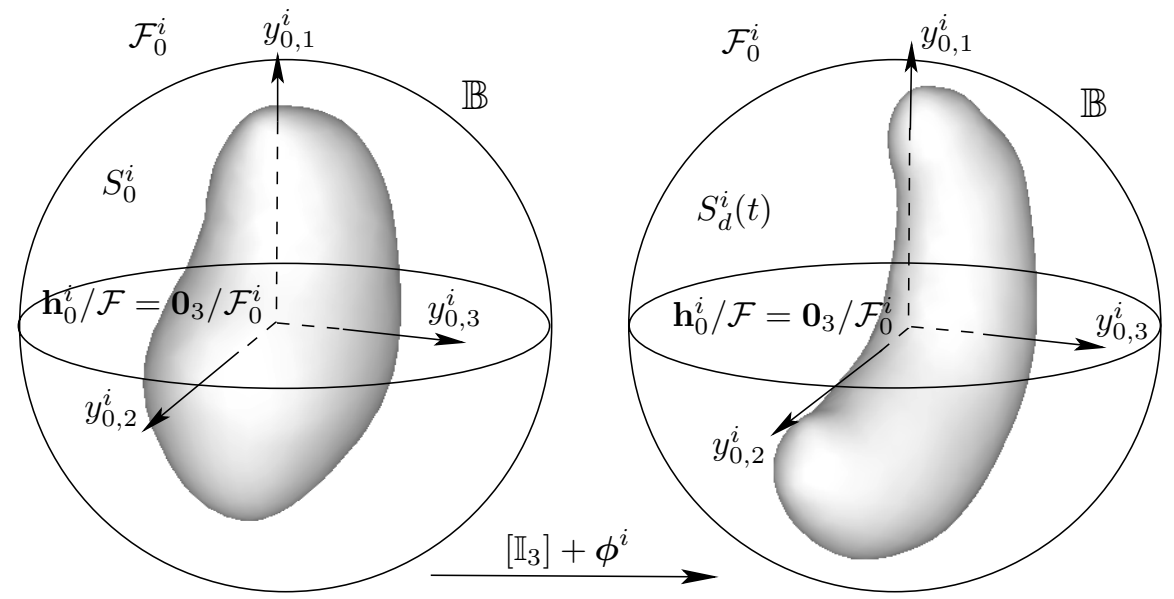

Figure 1 . The deformation of the $i$-th body is given in the frame $\mathcal{F}_{0}^{i}$ by a smooth function $\phi^{i}$ such that $\left[\mathbb{I}_{3}\right]+\phi^{i}$ maps $\mathbb{B}$ onto $\mathbb{B}$.

therefore have with the notations above: $\mathbf{y}^{i}(t)=\mathbf{\Phi}\left(t, \mathbf{y}_{0}^{i}\right)$ and $S_{d}^{i}(t)=\mathbf{\Phi}^{i}\left(t, S_{0}^{i}\right)$. The coordinates of $M^{i}(t)$ in the inertial frame $\mathcal{F}$ read:

$$
\mathbf{x}^{i}(t)=\left[R^{i}(t)\right] \Phi^{i}\left(t, \mathbf{x}_{0}^{i}-\mathbf{h}_{0}^{i}\right)+\mathbf{h}^{i}(t),
$$

and we define the following map using the partition $\cup_{i=1}^{n} S_{0}^{i}$ of $S_{0}$ :

$$
\begin{aligned}
\boldsymbol{\Lambda}:[0, \infty) \times S_{0} & \rightarrow \mathbb{R}^{3} \\
(t, \mathbf{x}) & \mapsto\left[R^{i}(t)\right] \mathbf{\Phi}^{i}\left(t, \mathbf{x}-\mathbf{h}_{0}^{i}\right)+\mathbf{h}^{i}(t), \text { if } \mathbf{x} \in S_{0}^{i} .
\end{aligned}
$$

We denote $\phi^{i}:=\Phi^{i}-\left[\mathbb{I}_{3}\right]$ and $\dot{\phi}^{i}:=\partial_{t} \phi^{i}$. Let $\mathbb{B}$ be a ball in $\mathbb{R}^{3}$, centered at the origin an large enough to contain all the initial regions $S_{0}^{i}-\mathbf{h}_{0}^{i}$ for $i=1, \ldots, n$. We assume that $\phi^{i}=\left(\phi_{1}^{i}, \phi_{2}^{i}, \phi_{3}^{i}\right)^{T} \in C_{0}^{m}\left(\mathbb{B}, \mathbb{R}^{3}\right)(m \geq 0)$, the space of the functions from $\mathbb{R}^{3}$ into $\mathbb{R}^{3}, m$-times continuously differentiable and that vanish outside of $\overline{\mathbb{B}}$. Differentiating (2.1) with respect to $t$, we obtain: $\dot{\mathbf{x}}^{i}(t)=\left[\dot{R}^{i}(t)\right] \Phi^{i}\left(t, \mathbf{x}_{0}^{i}-\mathbf{h}_{0}^{i}\right)+$ 


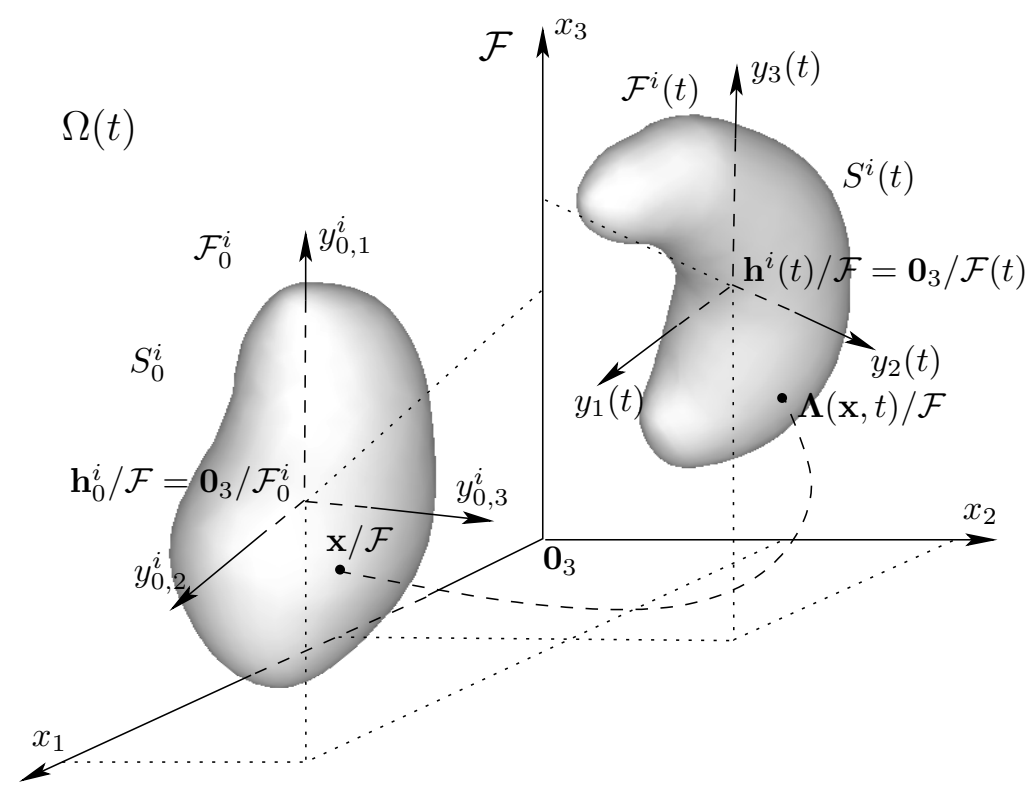

Figure 2. The rigid body $S^{i}(t)$ and the fluid domain $\Omega(t)$. The frames $\mathcal{F}_{0}^{i}$ and $\mathcal{F}$ are fixed whereas $\mathcal{F}^{i}(t)$ moves with the $i$-th swimmer. The coordinates of a material point that we follow in his motion is $\mathbf{x}$ at time $t=0$ in $\mathcal{F}$ and $\boldsymbol{\Lambda}(t, \mathbf{x})$ in the same frame at time $t>0$.

$\left[R^{i}(t)\right] \dot{\boldsymbol{\phi}}^{i}\left(t, \mathbf{x}_{0}^{i}-\mathbf{h}_{0}^{i}\right)+\dot{\mathbf{h}}^{i}(t)$. Combining this identity with (2.1), it comes:

$$
\dot{\mathbf{x}}^{i}(t)=\left[\dot{R}^{i}(t)\right]\left[R^{i}(t)\right]^{T}\left(\mathbf{x}^{i}(t)-\mathbf{h}^{i}(t)\right)+\dot{\mathbf{h}}^{i}(t)+\left[R^{i}(t)\right] \dot{\boldsymbol{\phi}}^{i}\left(t, \boldsymbol{\Lambda}^{-1}\left(t, \mathbf{x}^{i}(t)\right)-\mathbf{h}_{0}^{i}\right) .
$$

The matrix $\left[\dot{R}^{i}(t)\right]\left[R^{i}(t)\right]^{T}$ being skew symmetric, there exists a vector $\boldsymbol{\omega}^{i}(t)$ such that $\left[\dot{R}^{i}(t)\right]\left[R^{i}(t)\right]^{T}\left(\mathbf{x}^{i}(t)-\mathbf{h}^{i}(t)\right)=\boldsymbol{\omega}^{i}(t) \wedge\left(\mathbf{x}^{i}(t)-\mathbf{h}^{i}(t)\right)$ and we define, for all $\mathbf{x} \in S(t)$, the Eulerian deformation velocity $\mathbf{v}_{d}^{i}$ and the Eulerian rigid velocity $\mathbf{v}_{r}^{i}$ by:

$$
\begin{aligned}
& \mathbf{v}_{d}^{i}(t, \mathbf{x}):= \begin{cases}{\left[R^{i}(t)\right] \dot{\boldsymbol{\phi}}^{i}\left(t, \mathbf{\Lambda}^{-1}\left(t, \mathbf{x}^{i}\right)-\mathbf{h}_{0}^{i}\right)} & \text { if } \mathbf{x} \in S^{i}(t), \\
\mathbf{0}_{3} & \text { if } \mathbf{x} \in \cup_{j \neq i} S^{j}(t),\end{cases} \\
& \mathbf{v}_{r}^{i}(t, \mathbf{x}):= \begin{cases}\boldsymbol{\omega}^{i}(t) \wedge\left(\mathbf{x}^{i}-\mathbf{h}^{i}(t)\right)+\dot{\mathbf{h}}^{i}(t) & \text { if } \mathbf{x} \in S^{i}(t), \\
\mathbf{0}_{3} & \text { if } \mathbf{x} \in \cup_{j \neq i} S^{j}(t) .\end{cases}
\end{aligned}
$$

The Eulerian velocity in $S^{i}(t)$ is therefore:

$$
\mathbf{v}^{i}(t, \mathbf{x})=\mathbf{v}_{r}^{i}(t, \mathbf{x})+\mathbf{v}_{d}^{i}(t, \mathbf{x}), \quad \forall t \geq 0 .
$$

We denote $\rho_{S}(t, \mathbf{x})$ the density of the bodies, defined for all $\mathbf{x} \in S(t)$ and for all $\mathbf{x} \in S_{0}$ we set $\rho_{S}^{0}(\mathbf{x}):=\rho_{S}(\mathbf{x}, 0)$. The principle of conservation of mass leads to the identity:

$$
\rho_{S}(t, \mathbf{x})=\rho_{S}^{0}\left(\boldsymbol{\Lambda}^{-1}(t, \mathbf{x})\right)\left|\operatorname{det}\left[D \boldsymbol{\Lambda}^{-1}(t, \mathbf{x})\right]\right|, \quad \forall t \geq 0, \forall \mathbf{x} \in S(t) .
$$

We assume that the swimmers are self-propelled, which means that their deformations are due to inner forces only. The principle of conservation of linear and 
angular momenta ensures that, for all time $t$ and all $i=1, \ldots, n$ :

$$
\int_{S^{i}(t)} \rho_{S}(t, \mathbf{x}) \mathbf{v}_{d}^{i}(t, \mathbf{x}) \mathrm{d} \mathbf{x}=\mathbf{0}_{3} \quad \text { and } \quad \int_{S^{i}(t)} \rho_{S}(t, \mathbf{x})\left(\mathbf{x}-\mathbf{h}^{i}\right) \wedge \mathbf{v}_{d}^{i}(t, \mathbf{x}) \mathrm{d} \mathbf{x}=\mathbf{0}_{3}
$$

or equivalently, upon a change of variables and taking into account (2.5):

$$
\begin{gathered}
\int_{S_{0}^{i}} \rho_{S}^{0}(\mathbf{x}) \dot{\phi}^{i}\left(t, \mathbf{x}-\mathbf{h}_{0}^{i}\right) \mathrm{d} \mathbf{x}=\mathbf{0}_{3} \\
\int_{S_{0}^{i}} \rho_{S}^{0}(\mathbf{x})\left(\mathbf{x}+\phi^{i}\left(t, \mathbf{x}-\mathbf{h}_{0}^{i}\right)\right) \wedge \dot{\phi}^{i}\left(t, \mathbf{x}-\mathbf{h}_{0}^{i}\right) \mathrm{d} \mathbf{x}=\mathbf{0}_{3} .
\end{gathered}
$$

When the domain of the fluid $\Omega(t)$ is bounded and since the fluid is incompressible, both quantities $\int_{\Omega(t)} \mathrm{d} \mathbf{x}$ and $\int_{S(t)} \mathrm{d} \mathbf{x}$ do not depend on time. By virtue of Theorem 5.2 .2 page 172 in Ref. [18], we deduce that, for all time $t$ :

$$
\sum_{i=1}^{n} \int_{S^{i}(t)} \operatorname{div}\left(\mathbf{v}_{d}^{i}(t, \mathbf{x})\right) \mathrm{d} \mathbf{x}=0
$$

or equivalently, upon a change of variables:

$$
\sum_{i=1}^{n} \int_{S_{0}^{i}} \operatorname{div}\left(\dot{\phi}^{i}\left(t, \mathbf{x}-\mathbf{h}_{0}^{i}\right)\right)\left|\operatorname{det}\left(\left[\mathbb{I}_{3}\right]+\left[D \phi^{i}\left(t, \mathbf{x}-\mathbf{h}_{0}^{i}\right)\right]\right)\right|=0 .
$$

Relations (2.7) mean that the volume of the fluid and the volume of the swimmers are globally preserved in the motion; see $\mathrm{C}$ for a precise definition of the deformations set $\mathcal{D}^{n, m}$.

According to relation (2.1), the location and the shape of the $i$-th body are given by a pair $\mathbf{Q}^{i}:=\left(\left[R^{i}\right], \mathbf{h}^{i}\right) \in S O(3) \times \mathbb{R}^{3}$ and a function $\phi^{i}:=\left(\phi_{1}^{i}, \phi_{2}^{i}, \phi_{3}^{i}\right)^{T} \in$ $C_{0}^{m}\left(\mathbb{B}, \mathbb{R}^{3}\right)$. We write $\mathbf{Q}:=\left(\mathbf{Q}^{1}, \ldots, \mathbf{Q}^{n}\right):=\left(\left(\left[R^{1}\right], \mathbf{h}^{1}\right), \ldots,\left(\left[R^{n}\right], \mathbf{h}^{n}\right)\right) \in(S O(3) \times$ $\left.\mathbb{R}^{3}\right)^{n}$, and $\phi:=\left(\phi^{1}, \ldots, \phi^{n}\right)^{T}=\left(\phi_{1}^{1}, \phi_{2}^{1}, \phi_{3}^{1}, \ldots, \phi_{1}^{n}, \phi_{2}^{n}, \phi_{3}^{n}\right)^{T} \in C_{0}^{m}\left(\mathbb{B}, \mathbb{R}^{3}\right)^{n}$. In $\mathrm{C}$, we define the $6 n$ - dimensional manifold $\mathcal{Q}_{a l}^{n, m}$ of the swimmers allowable position and shape. The pairs $(\mathbf{Q}, \boldsymbol{\phi})$ of $\mathcal{Q}_{a l}^{n, m}$ are called the global coordinates in the sequel. Building a local chart, we define the open set $\mathfrak{Q}_{a l}^{n, m} \subset \mathbb{R}^{6 n} \times C_{0}^{m}\left(\mathbb{B}, \mathbb{R}^{3}\right)^{n}$ of the local coordinates, denoted $(\mathbf{q}, \boldsymbol{\phi})$. Also in $\mathrm{C}$, we introduce the tangent bundles $T \mathcal{Q}_{a l}^{n, m} \ni(\mathbf{Q}, \phi, \dot{\mathbf{Q}}, \dot{\boldsymbol{\phi}})$ and $T \mathfrak{Q}_{a l}^{n, m} \ni(\mathbf{q}, \boldsymbol{\phi}, \dot{\mathbf{q}}, \dot{\boldsymbol{\phi}})$.

2.2. Lagrangian mechanics. The equations of motion are obtained by applying the least action principle of Lagrangian mechanics. In local coordinates, the Lagrangian reads:

$$
\begin{aligned}
\mathcal{L}: T \mathfrak{Q}_{a l}^{n, m} & \rightarrow \mathbb{R} \\
(\mathbf{q}, \boldsymbol{\phi}, \dot{\mathbf{q}}, \dot{\boldsymbol{\phi}}) & \mapsto \mathcal{L}(\mathbf{q}, \boldsymbol{\phi}, \dot{\mathbf{q}}, \dot{\boldsymbol{\phi}}) .
\end{aligned}
$$

It is obtained as the difference between the total kinetic energy of the system and the potential energy due to the buoyant force acting on the bodies. It will be computed explicitly in Section 3. The deformation $\phi$ (hence $\dot{\phi}$ too) of the swimmers being given, the vectorial Euler-Lagrange equation of the motion reads, following V. I. Arnold[3], page 56:

$$
\frac{d}{d t} \partial_{\dot{\mathbf{q}}} \mathcal{L}(\mathbf{q}, \boldsymbol{\phi}, \dot{\mathbf{q}}, \dot{\boldsymbol{\phi}})-\partial_{\mathbf{q}} \mathcal{L}(\mathbf{q}, \boldsymbol{\phi}, \dot{\mathbf{q}}, \dot{\boldsymbol{\phi}})=\mathbf{0}_{6 n}
$$


Since $\mathcal{L}$ depends on time only through the variables $(\mathbf{q}, \boldsymbol{\phi}, \dot{\mathbf{q}}, \dot{\boldsymbol{\phi}})$ and if $\mathcal{L}$ is smooth enough, we can apply the chain rule and rewrite (2.9) under the form of a second order ODE in q:

$$
\begin{aligned}
\left\langle\partial_{\dot{\mathbf{q}} \dot{\mathbf{q}}}^{2} \mathcal{L}(\mathbf{q}, \boldsymbol{\phi}, \dot{\mathbf{q}}, \dot{\boldsymbol{\phi}}), \ddot{\mathbf{q}}\right\rangle & +\left\langle\partial_{\mathbf{q} \dot{\mathbf{q}}}^{2} \mathcal{L}(\mathbf{q}, \boldsymbol{\phi}, \dot{\mathbf{q}}, \dot{\boldsymbol{\phi}}), \dot{\mathbf{q}}\right\rangle+\left\langle\partial_{\dot{\boldsymbol{\phi}} \dot{\mathbf{q}}}^{2} \mathcal{L}(\mathbf{q}, \boldsymbol{\phi}, \dot{\mathbf{q}}, \dot{\boldsymbol{\phi}}), \ddot{\boldsymbol{\phi}}\right\rangle \\
& +\left\langle\partial_{\dot{\phi} \dot{\mathbf{q}}}^{2} \mathcal{L}(\mathbf{q}, \boldsymbol{\phi}, \dot{\mathbf{q}}, \dot{\boldsymbol{\phi}}), \dot{\boldsymbol{\phi}}\right\rangle-\partial_{\mathbf{q}} \mathcal{L}(\mathbf{q}, \boldsymbol{\phi}, \dot{\mathbf{q}}, \dot{\boldsymbol{\phi}})=\mathbf{0}_{6 n} .
\end{aligned}
$$

2.3. Statement of the main results. The first result concerns the existence and uniqueness of local solutions for the ODE $(2.10)$. We denote $\phi_{0}:=\left(\left[\mathbb{O}_{3}\right], \ldots,\left[\mathbb{O}_{3}\right]\right) \in$ $C_{0}^{m}\left(\mathbb{B}, \mathbb{R}^{3}\right)^{n}$.

Theorem 1 (Existence and uniqueness of local solutions). Assume that $\partial \Omega$ is Lipschitz continuous and $m=1$ in (2.8). Then the Lagrangian (2.8) is analytic ${ }^{1}$ with respect to $(\mathbf{q}, \boldsymbol{\phi}, \dot{\mathbf{q}}, \dot{\boldsymbol{\phi}}) \in T \mathfrak{Q}_{a l}^{n, m}$ and all the terms in the ODE (2.10) make sense. For any initial positions and shapes $S_{0}$ of the bodies, any $\dot{\mathbf{q}}_{0} \in \mathbb{R}^{6 n}$ and any given deformations $t \in[0, \infty) \mapsto \phi(t)=\left(\phi^{1}(t), \ldots, \phi^{n}(t)\right)^{T} \in C_{0}^{1}\left(\mathbb{B}, \mathbb{R}^{3}\right)^{n}$ of class $C^{k}(2 \leq k \leq \infty)$ (respectively analytic) such that $\phi(0)=\phi_{0}$ and $(\phi(t), \dot{\phi}(t)) \in$ $\mathcal{D}^{n, 1}$ for all $t \geq 0$, there exists $T>0$ and a unique twice differentiable function $\mathbf{q}^{*}:[0, T) \rightarrow \mathbb{R}^{6 n}$, for which $\mathbf{q}^{*}(0)=\mathbf{0}_{6 n}, \dot{\mathbf{q}}^{*}(0)=\dot{\mathbf{q}}_{0}$ and that solves the ODE (2.10). Moreover, the function $\mathbf{q}^{*}$ is of class $C^{k}$ (respectively analytic) and $\left(\left(\mathbf{q}^{*}(t), \boldsymbol{\phi}(t)\right),\left(\dot{\mathbf{q}}^{*}(t), \dot{\phi}(t)\right) \in T \mathfrak{Q}_{a l}^{n, 1}\right.$ for all $0 \leq t<T$.

Combining this theorem with an a priori energy estimate and using local charts, we will show:

Corollary 1 (Existence and uniqueness of global solutions). Assume that $\partial \Omega$ is Lipschitz continuous. Then for any initial position and shape $S_{0}$ of the bodies, any collection of $n 3 \times 3$ skew-symmetric matrices $\left[\dot{R}_{0}^{1}\right], \ldots,\left[\dot{R}_{0}^{n}\right]$, any initial velocities $\left(\dot{\mathbf{h}}_{0}^{1}, \ldots, \dot{\mathbf{h}}_{0}^{n}\right) \in \mathbb{R}^{3 n}$ and any given deformations $t \in[0, \infty) \mapsto \phi(t)=\left(\phi^{1}(t), \ldots\right.$, $\left.\phi^{n}(t)\right)^{T} \in C_{0}^{1}\left(\mathbb{B}, \mathbb{R}^{3}\right)^{n}$ of class $C^{k}(2 \leq k \leq \infty)$ (respectively analytic) such that $\phi(0)=\phi_{0}$ and $(\phi(t), \dot{\phi}(t)) \in \mathcal{D}^{n, 1}$ for all $t \geq 0$, there exists $0<T^{*} \leq \infty$ and a unique twice differentiable function:

$$
\begin{aligned}
\mathbf{Q}^{*}:\left[0, T^{*}\right) & \rightarrow\left(S O(3) \times \mathbb{R}^{3}\right)^{n} \\
t & \mapsto\left(\left(\left[R^{1}(t)\right], \mathbf{h}^{1}(t)\right), \ldots,\left(\left[R^{n}(t)\right], \mathbf{h}^{n}(t)\right)\right),
\end{aligned}
$$

for which $\mathbf{Q}^{*}(0)=\left(\left[\mathbb{I}_{3}\right], \mathbf{h}_{0}^{1}, \ldots,\left[\mathbb{I}_{3}\right], \mathbf{h}_{0}^{n}\right), \dot{\mathbf{Q}}^{*}(0)=\left(\left[\dot{R}_{0}^{1}\right], \dot{\mathbf{h}}_{0}^{1}, \ldots,\left[\dot{R}_{0}^{n}\right], \dot{\mathbf{h}}_{0}^{n}\right)$ and such that the coordinates $\mathbf{x}^{i}(t)$ in $\mathcal{F}$ of a material point, occupying position $\mathbf{x}_{0}^{i} \in S_{0}^{i}$ at instant $t=0$, be given by (2.1). Furthermore, the function $\mathbf{Q}^{*}$ is of class $C^{k}$ (respectively analytic) and $\left(\left(\mathbf{Q}^{*}(t), \phi(t)\right),\left(\dot{\mathbf{Q}}^{*}(t), \dot{\phi}(t)\right) \in T \mathcal{Q}_{\text {al }}^{n, 1}\right.$ for all $0 \leq t<T^{*}$. Time $T^{*}$ corresponds to the time of a collision between two bodies or between a body with the fluid's domain boundary. If there is no collision $T^{*}=\infty$.

Specifying $\phi$ to be the null function, the bodies behave like a set of $n$ solids in a perfect fluid. We deduce straightforwardly from Corollary 1:

Corollary 2 (Motion of solids in a perfect fluid). Assume that $\partial \Omega$ is Lipschitz continuous. Then, the $6 n$ degrees of freedom of a set of $n$ solids subject or not to the buoyant force in a perfect fluid are analytic functions of $t$ up to the time

\footnotetext{
${ }^{1}$ We refer to Ref. [8], $\S 4$ for the definition of analytic functions of several variables in Banach spaces.
} 
of the first collision between two solids of between a solid with the fluid's domain boundary.

This result improves the one obtained in Ref. [12] in which we prove under the same assumptions that the degrees of freedom of the solids are infinitely continuously differentiable. In the same paper, we also prove that collisions (i.e. contacts with non zero relative velocity) can occur. This situation heavily contrasts to the one encountered for viscous fluids; see Ref. [19], [20], [33] and [38]. Considering again deformable bodies and assuming additional regularity for the fluid's boundary and for the deformations, we can make the ODE (2.10) explicit. We give the result in the case of a swimmer alone and a bounded fluid's domain:

Theorem 2. Assume that $\Omega$ is bounded, $\partial \Omega$ is of class $C^{1,1}, n=1$ and $m=2$ in (2.8) and that $t \in[0, \infty) \mapsto \phi(t) \in C_{0}^{2}\left(\mathbb{B}, \mathbb{R}^{3}\right)$ is of class $C^{2}$ with $(\phi, \dot{\phi}) \in \mathcal{D}_{a l}^{1,2}$ for all $t \geq 0$ and $\phi(0)=\left[\mathbb{O}_{3}\right]$. Then for any $\dot{\mathbf{q}}_{0} \in \mathbb{R}^{6}$, the $C^{2}$-function $\mathbf{q}^{*}:[0, T) \rightarrow \mathbb{R}^{6}$ of Theorem 1 solves the following second order non-linear ODE with initial data $\left(\mathbf{q}^{*}(0), \dot{\mathbf{q}}^{*}(0)\right)=\left(\mathbf{0}_{6}, \dot{\mathbf{q}}_{0}\right)$ :

$$
\begin{aligned}
&\left(\left[K_{S}(t, \mathbf{q})\right]+\left[K_{F}(t, \mathbf{q})\right]\right) \ddot{\mathbf{q}}+\left\langle\llbracket T_{s y m}^{S / r}(t, \mathbf{q}) \rrbracket+\right.\left.\llbracket T_{\text {skew }}^{S / r}(t, \mathbf{q}) \rrbracket, \dot{\mathbf{q}}, \dot{\mathbf{q}}\right\rangle \\
&+\left\langle\llbracket T_{\text {sym }}^{F / r}(t, \mathbf{q}) \rrbracket+\llbracket T_{\text {skew }}^{F / r}(t, \mathbf{q}) \rrbracket, \dot{\mathbf{q}}, \dot{\mathbf{q}}\right\rangle \\
&+\left[M_{\text {sym }}^{S / d}(t, \mathbf{q})\right] \dot{\mathbf{q}}+\left(\left[M_{\text {sym }}^{F / d}(t, \mathbf{q})\right]+\right. {\left.\left[M_{\text {skew }}^{F / d}(t, \mathbf{q})\right]\right) \dot{\mathbf{q}} } \\
&+\mathbf{F}^{F / d}(t, \mathbf{q})+\mathbf{F}^{b}(t, \mathbf{q})=\mathbf{0}_{6} .
\end{aligned}
$$

The $6 \times 6$ symmetric matrices $\left[K_{S}(t, \mathbf{q})\right],\left[K_{F}(t, \mathbf{q})\right]\left(\right.$ nota: $\left[K_{S}(t, \mathbf{q})\right]+\left[K_{F}(t, \mathbf{q})\right]$ is the virtual mass matrix) and $\left[M_{s y m}^{S / d}(t, \mathbf{q})\right]$ are defined in the Appendix by (D.2), the $6 \times 6 \times 6$ third-rank tensors $\llbracket T_{s y m}^{S / r}(t, \mathbf{q}) \rrbracket, \llbracket T_{\text {skew }}^{S / r}(t, \mathbf{q}) \rrbracket, \llbracket T_{s y m}^{F / r}(t, \mathbf{q}) \rrbracket$ and $\llbracket T_{s y m}^{F / r}(t, \mathbf{q}) \rrbracket$ by (D.3), the $6 \times 6$ matrices $\left[M_{\text {sym }}^{F / d}(t, \mathbf{q})\right]$ and $\left[M_{\text {skew }}^{F / d}(t, \mathbf{q})\right]$ by (D.4) and the 6 -length column vectors $\mathbf{F}^{F / d}(t, \mathbf{q})$ and $\mathbf{F}^{b}(t, \mathbf{q})$ by (D.5).

The last term $\mathbf{F}^{b}(t, \mathbf{q})$ of the left hand side of (2.11) is the buoyant force. Choosing for $\phi$ the identically null function, the body behaves like a solid. In this case, all the quantities $\left[K_{S}(t, \mathbf{q})\right], \llbracket T_{\text {sym }}^{S / r}(t, \mathbf{q}) \rrbracket, \llbracket T_{\text {skew }}^{S / r}(t, \mathbf{q}) \rrbracket, \llbracket T_{s y m}^{F / r}(t, \mathbf{q}) \rrbracket, \llbracket T_{\text {skew }}^{F / r}(t, \mathbf{q}) \rrbracket$ and $\mathbf{F}^{b}(t, \mathbf{q})$ depend on times only through the variable $\mathbf{q}$. Dropping $t$ in the notations:

$$
\left[K_{S}(\mathbf{q})\right] \ddot{\mathbf{q}}+\left\langle\llbracket T_{\text {sym }}^{S / r}(\mathbf{q}) \rrbracket+\llbracket T_{\text {skew }}^{S / r}(\mathbf{q}) \rrbracket, \dot{\mathbf{q}}, \dot{\mathbf{q}}\right\rangle=\mathbf{0}_{6},
$$

is the Euler-Lagrange equation for the free motion of a rigid body in the absence of fluid and of gravity. In the same way, the Euler-Lagrange equation for the same solid immersed in a bounded weighted fluid is:

$$
\begin{aligned}
& \left(\left[K_{S}(\mathbf{q})\right]+\left[K_{F}(\mathbf{q})\right]\right) \ddot{\mathbf{q}}+\left\langle\llbracket T_{\text {sym }}^{S / r}(\mathbf{q}) \rrbracket+\llbracket T_{\text {skew }}^{S / r}(\mathbf{q}) \rrbracket, \dot{\mathbf{q}}, \dot{\mathbf{q}}\right\rangle \\
& \quad+\left\langle\llbracket T_{\text {sym }}^{F / r}(\mathbf{q}) \rrbracket+\llbracket T_{\text {skew }}^{F / r}(\mathbf{q}) \rrbracket, \dot{\mathbf{q}}, \dot{\mathbf{q}}\right\rangle+\mathbf{F}^{b}(\mathbf{q})=\mathbf{0}_{6} .
\end{aligned}
$$

\section{Computation of the Lagrangian of the System}

Keeping the notation $\boldsymbol{\Phi}^{i}:=\left[\mathbb{I}_{3}\right]+\boldsymbol{\phi}^{i}$ for all $i=1, \ldots, n$, we set $\boldsymbol{\Phi}:=\left(\boldsymbol{\Phi}^{1}, \ldots, \boldsymbol{\Phi}^{n}\right)^{T}$ : $\mathbb{R}^{3} \rightarrow \mathbb{R}^{3 n}$. In local coordinates (see $\mathrm{C}$ ), we rewrite $(2.1)$ :

$$
\mathbf{x}^{i}\left(\mathbf{q}^{i}, \boldsymbol{\phi}^{i}\right)=\mathcal{R}\left(\boldsymbol{\alpha}^{i}\right) \boldsymbol{\Phi}^{i}\left(\mathbf{x}_{0}^{i}-\mathbf{h}_{0}^{i}\right)+\mathbf{h}^{i} .
$$


The domain of the $i$-th swimmer is $S^{i}\left(\mathbf{q}^{i}, \boldsymbol{\phi}^{i}\right):=\mathcal{R}\left(\boldsymbol{\alpha}^{i}\right) \Phi^{i}\left(S_{0}^{i}-\mathbf{h}_{0}^{i}\right)+\mathbf{h}^{i}$ and $S(\mathbf{q}, \boldsymbol{\phi}):=\cup_{i=1}^{n} S^{i}\left(\mathbf{q}^{i}, \boldsymbol{\phi}^{i}\right), \Gamma_{2}^{i}(\mathbf{q}, \boldsymbol{\phi}):=\partial S^{i}(\mathbf{q}, \boldsymbol{\phi})$ and $\Gamma_{2}(\mathbf{q}, \boldsymbol{\phi}):=\partial S(\mathbf{q}, \boldsymbol{\phi})$. The definition (2.2) of the function $\boldsymbol{\Lambda}$ turns into:

$$
\begin{aligned}
\boldsymbol{\Lambda}: \mathfrak{Q}_{a l}^{n, m} \times S_{0} & \rightarrow \mathbb{R}^{3} \\
((\mathbf{q}, \boldsymbol{\phi}), \mathbf{x}) & \mapsto \mathcal{R}\left(\boldsymbol{\alpha}^{i}\right) \Phi^{i}\left(\mathbf{x}-\mathbf{h}_{0}^{i}\right)+\mathbf{h}^{i}, \quad \text { if } \mathbf{x} \in S_{0}^{i} .
\end{aligned}
$$

Since the compact regions $S_{0}^{i}$ and the boundary $\Gamma_{1}$ do not touch or overlap each other, we can extend the function $\boldsymbol{\Lambda}$ to $\mathfrak{Q}_{a l}^{n, m} \times \mathbb{R}^{3}$ in such a way that $\left.\boldsymbol{\Lambda}((\mathbf{q}, \boldsymbol{\phi}), \cdot)\right|_{\Gamma_{1}}=$ $\left[\mathbb{I}_{3}\right]$ for all $(\mathbf{q}, \phi) \in \mathfrak{Q}_{a l}^{n, m}$, using an appropriate $C^{\infty}$-partition of unity. The resulting function $\boldsymbol{\Lambda}((\mathbf{q}, \phi), \cdot)$ is a $C^{m}$-diffeormorphism from $\mathbb{R}^{3}$ onto $\mathbb{R}^{3}$ for all $(\mathbf{q}, \phi) \in \mathfrak{Q}_{a l}^{n, m}$ and the domain of the fluid is $\Omega(\mathbf{q}, \phi):=\boldsymbol{\Lambda}((\mathbf{q}, \phi), \Omega)$. We rewrite the Eulerian velocities (2.3):

$$
\mathbf{v}_{d}^{i}(\mathbf{q}, \boldsymbol{\phi}, \dot{\boldsymbol{\phi}}, \mathbf{x}):= \begin{cases}\mathcal{R}\left(\boldsymbol{\alpha}^{i}\right) \dot{\boldsymbol{\phi}}^{i}\left(\boldsymbol{\Lambda}^{-1}(\mathbf{q}, \boldsymbol{\phi}, \mathbf{x})-\mathbf{h}_{0}^{i}\right) & \text { if } \mathbf{x} \in S^{i}\left(\mathbf{q}^{i}, \boldsymbol{\phi}^{i}\right), \\ \mathbf{0}_{3} & \text { if } \mathbf{x} \in S(\mathbf{q}, \phi) \backslash S^{i}\left(\mathbf{q}^{i}, \boldsymbol{\phi}^{i}\right),\end{cases}
$$

and

$$
\mathbf{v}_{r}^{i}(\mathbf{q}, \boldsymbol{\phi}, \dot{\mathbf{q}}, \mathbf{x}):= \begin{cases}{\left[\omega\left(\boldsymbol{\alpha}^{i}\right)\right] \dot{\boldsymbol{\alpha}}^{i} \wedge\left(\mathbf{x}-\mathbf{h}^{i}\right)+\dot{\mathbf{h}}^{i}} & \text { if } \mathbf{x} \in S^{i}\left(\mathbf{q}^{i}, \phi^{i}\right), \\ \mathbf{0}_{3} & \text { if } \mathbf{x} \in S(\mathbf{q}, \phi) \backslash S^{i}\left(\mathbf{q}^{i}, \phi^{i}\right),\end{cases}
$$

the matrix $[\omega]$ and the vectors $\boldsymbol{\omega}_{k}, k=1,2,3$, being defined in A. We denote merely $\left[\omega^{i}\right]$ for $\left[\omega\left(\boldsymbol{\alpha}^{i}\right)\right]$ and $\boldsymbol{\omega}_{k}^{i}$ for $\boldsymbol{\omega}_{k}^{i}\left(\boldsymbol{\alpha}^{i}\right)$. The rigid velocity $\mathbf{v}_{r}^{i}(\mathbf{q}, \boldsymbol{\phi}, \dot{\mathbf{q}}, \mathbf{x})$ of the body can be decomposed into $\mathbf{v}_{r}^{i}(\mathbf{q}, \boldsymbol{\phi}, \dot{\mathbf{q}}, \mathbf{x})=\sum_{k=1}^{6} \mathbf{v}_{r}^{i, k}(\mathbf{q}, \boldsymbol{\phi}, \mathbf{x}) \dot{q}_{k}^{i}$, where we have set:

$$
\mathbf{v}_{r}^{i, k}(\mathbf{q}, \boldsymbol{\phi}, \mathbf{x}):=\left\{\begin{array}{lll}
\left\{\boldsymbol{\omega}_{k}^{i} \wedge\left(\mathbf{x}-\mathbf{h}^{i}\right)\right. & \text { if } k=1,2,3, \\
\mathbf{e}_{k-3} & \text { if } k=4,5,6, & \text { if } \mathbf{x} \in S^{i}\left(\mathbf{q}^{i}, \boldsymbol{\phi}^{i}\right), \\
\mathbf{0}_{3} & \text { if } \mathbf{x} \in \cup_{j \neq i} S^{j}\left(\mathbf{q}^{j}, \boldsymbol{\phi}^{j}\right) .
\end{array}\right.
$$

The kinetic energy of the $i$-th body reads:

$$
\mathcal{K}_{S^{i}}\left(\mathbf{q}^{i}, \phi^{i}, \dot{\mathbf{q}}^{i}, \dot{\phi}^{i}\right)=\frac{1}{2} \int_{S^{i}\left(\mathbf{q}^{i}, \phi^{i}\right)} \rho_{S}\left|\left[\omega^{i}\right] \dot{\boldsymbol{\alpha}}^{i} \wedge\left(\mathbf{x}-\mathbf{h}^{i}\right)+\dot{\mathbf{h}}^{i}+\mathbf{v}_{d}^{i}\right|^{2} \mathrm{~d} \mathbf{x}
$$

Taking into account the properties (2.6) and (2.7) of $\phi^{i}$ and $\dot{\phi}^{i}$, the expression above can be expanded as follows:

$$
\mathcal{K}_{S^{i}}=\frac{1}{2} \dot{\boldsymbol{\alpha}}^{i} \cdot\left[\omega^{i}\right]^{T}\left[I^{i}\left(\boldsymbol{\alpha}^{i}, \boldsymbol{\phi}^{i}\right)\right]\left[\omega^{i}\right] \dot{\boldsymbol{\alpha}}^{i}+\frac{1}{2} m^{i}\left|\dot{\mathbf{h}}^{i}\right|^{2}+\frac{1}{2} \int_{S_{0}^{i}} \rho_{S}^{0}\left|\dot{\boldsymbol{\phi}}^{i}\left(\mathbf{x}-\mathbf{h}_{0}^{i}\right)\right|^{2} \mathrm{~d} \mathbf{x}
$$

where $\left.\left[I^{i}\left(\boldsymbol{\alpha}^{i}, \boldsymbol{\phi}\right)\right)\right]$ is the inertia tensor of the $i$-th body. It is classically defined by:

$$
\left[I^{i}\left(\boldsymbol{\alpha}^{i}, \boldsymbol{\phi}^{i}\right)\right]:=\int_{S^{i}\left(\mathbf{q}^{i}, \boldsymbol{\phi}^{i}\right)} \underset{\rho_{S}}{ }(\mathbf{x})\left(\left|\mathbf{x}-\mathbf{h}^{i}\right|^{2}\left[\mathbb{I}_{3}\right]-\left(\mathbf{x}-\mathbf{h}^{i}\right) \otimes\left(\mathbf{x}-\mathbf{h}^{i}\right)\right) \mathrm{d} \mathbf{x},
$$

or equivalently, upon a change of variables by

$$
\left[I^{i}\left(\boldsymbol{\alpha}^{i}, \boldsymbol{\phi}\right)\right]=\mathcal{R}\left(\boldsymbol{\alpha}^{i}\right)\left[I_{0}^{i}\left(\phi^{i}\right)\right] \mathcal{R}\left(\boldsymbol{\alpha}^{i}\right)^{T}
$$

with:

$$
\left[I_{0}^{i}\left(\phi^{i}\right)\right]:=\int_{S_{0}^{i}} \rho_{S}^{0}\left(\left|\Phi^{i}\left(\mathbf{x}-\mathbf{h}_{0}^{i}\right)\right|^{2}\left[\mathbb{I}_{3}\right]-\boldsymbol{\Phi}^{i}\left(\mathbf{x}-\mathbf{h}_{0}^{i}\right) \otimes \boldsymbol{\Phi}^{i}\left(\mathbf{x}-\mathbf{h}_{0}^{i}\right)\right) \mathrm{d} \mathbf{x}
$$


Let $m^{i}$ be the mass of the $i$-th body. Setting the $6 n \times 6 n$ symmetric block-diagonal matrix:

$$
\left[K_{S}^{r}(\mathbf{q}, \phi)\right]:=\operatorname{diag}\left(\left[\omega^{i}\right]^{T}\left[I^{1}\right]\left[\omega^{i}\right], m^{1}\left[\mathbb{I}_{3}\right], \ldots,\left[\omega^{i}\right]^{T}\left[I^{n}\right]\left[\omega^{i}\right], m^{n}\left[\mathbb{I}_{3}\right]\right),
$$

and the scalar:

$$
K_{S}^{d}(\dot{\boldsymbol{\phi}}, \dot{\boldsymbol{\phi}}):=\sum_{i=1}^{n} \int_{S_{0}^{i}} \rho_{S}^{0}\left|\dot{\phi}^{i}\left(\mathbf{x}-\mathbf{h}_{0}^{i}\right)\right|^{2} \mathrm{~d} \mathbf{x}
$$

we obtain that the total kinetic energy of the bodies is $\mathcal{K}_{S}(\mathbf{q}, \boldsymbol{\phi}, \dot{\mathbf{q}}, \dot{\phi})=(1 / 2) \dot{\mathbf{q}}$. $\left[K_{S}^{r}(\mathbf{q}, \boldsymbol{\phi})\right] \dot{\mathbf{q}}+(1 / 2) K_{S}^{d}(\dot{\boldsymbol{\phi}}, \dot{\boldsymbol{\phi}})$. Let us compute now the kinetic energy of the fluid. The Eulerian velocity in an perfect fluid is given by $\mathbf{u}(t, \cdot)=\nabla \varphi(t, \cdot)^{T}$, where $\varphi(t, \cdot)$ is the harmonic potential. Using the local coordinates, we can write that $\varphi(t, \cdot)=$ $\varphi(\mathbf{q}, \boldsymbol{\phi}, \dot{\mathbf{q}}, \dot{\boldsymbol{\phi}}, \cdot)$ and the Kirchoff principle for potential flow allows us to decompose $\varphi$ into the form $\varphi(\mathbf{q}, \boldsymbol{\phi}, \dot{\mathbf{q}}, \dot{\boldsymbol{\phi}}, \cdot)=\sum_{i=1}^{n}\left(\sum_{k=1}^{6} \varphi_{r, k}^{i}(\mathbf{q}, \boldsymbol{\phi}, \cdot) \dot{q}_{k}^{i}+\varphi_{d}^{i}(\mathbf{q}, \boldsymbol{\phi}, \dot{\boldsymbol{\phi}}, \cdot)\right)$ in which the functions $\varphi_{r, k}^{i}(\mathbf{q}, \boldsymbol{\phi}, \cdot)$ are the potentials resulting from the rigid motion and $\varphi_{d}^{i}(\mathbf{q}, \phi, \dot{\boldsymbol{\phi}}, \cdot)$ are the potentials due the deformations of the swimmers. Each potential is harmonic in $\Omega(\mathbf{q}, \phi)$ and satisfies Neumann boundary conditions:

$$
\begin{aligned}
& \partial_{\mathbf{n}} \varphi_{r, k}^{i}(\mathbf{q}, \boldsymbol{\phi}, \cdot)=0 \text { on } \Gamma_{1}, \\
& \partial_{\mathbf{n}} \varphi_{r, k}^{i}(\mathbf{q}, \boldsymbol{\phi}, \cdot)=\mathbf{v}_{r}^{i, k} \cdot \mathbf{n} \text { on } \Gamma_{2}(\mathbf{q}, \boldsymbol{\phi}), \quad k=1, \ldots, 6,
\end{aligned}
$$

and

$$
\partial_{\mathbf{n}} \varphi_{d}^{i}(\mathbf{q}, \boldsymbol{\phi}, \dot{\boldsymbol{\phi}}, \cdot)=0 \text { on } \Gamma_{1}, \quad \partial_{\mathbf{n}} \varphi_{d}^{i}(\mathbf{q}, \boldsymbol{\phi}, \dot{\boldsymbol{\phi}}, \cdot)=\mathbf{v}_{d}^{i} \cdot \mathbf{n} \text { on } \Gamma_{2}(\mathbf{q}, \boldsymbol{\phi}) .
$$

The vector $\mathbf{n}$ is the unitary normal to $\partial \Omega(\mathbf{q}, \boldsymbol{\phi})$ directed toward the exterior of the fluid. Following our notation, we denote $\varphi_{r}^{i}:=\left(\varphi_{r, 1}^{i}, \ldots, \varphi_{r, 6}^{i}\right)^{T}: \Omega(\mathbf{q}, \boldsymbol{\phi}) \rightarrow \mathbb{R}^{6}$ and $\boldsymbol{\varphi}_{r}:=\left(\boldsymbol{\varphi}_{r}^{1}, \ldots, \varphi_{r}^{n}\right)^{T}: \Omega(\mathbf{q}, \boldsymbol{\phi}) \rightarrow \mathbb{R}^{6 n}$ whereas $\varphi_{d}:=\sum_{i=1}^{n} \varphi_{d}^{i}$ is a scalar function. Introducing $\rho_{F}$, the constant density of the fluid, the kinetic energy of the fluid is:

$$
\mathcal{K}_{F}(\mathbf{q}, \phi, \dot{\mathbf{q}}, \dot{\phi})=\frac{1}{2} \int_{\Omega(\mathbf{q}, \boldsymbol{\phi})} \rho_{F}|\mathbf{u}(\mathbf{q}, \boldsymbol{\phi}, \dot{\mathbf{q}}, \dot{\phi}, \mathbf{x})|^{2} \mathrm{~d} \mathbf{x} .
$$

Setting the $6 n \times 6 n$ symmetric matrix:

$$
\left[K_{F}^{r}(\mathbf{q}, \boldsymbol{\phi})\right]:=\int_{\Omega(\mathbf{q}, \boldsymbol{\phi})} \rho_{F}\left[D \boldsymbol{\varphi}_{r}(\mathbf{q}, \boldsymbol{\phi})\right]\left[D \boldsymbol{\varphi}_{r}(\mathbf{q}, \boldsymbol{\phi})\right]^{T} \mathrm{~d} \mathbf{x},
$$

the scalar:

$$
K_{F}^{d}(\mathbf{q}, \boldsymbol{\phi}, \dot{\phi}, \dot{\phi}):=\int_{\Omega(\mathbf{q}, \boldsymbol{\phi})} \rho_{F} \nabla \varphi_{d}(\mathbf{q}, \boldsymbol{\phi}, \dot{\phi}) \cdot\left(\nabla \varphi_{d}(\mathbf{q}, \boldsymbol{\phi}, \dot{\phi})\right)^{T} \mathrm{~d} \mathbf{x},
$$

and the $6 n$-length column vector:

$$
\mathbf{K}_{F}^{r, d}(\mathbf{q}, \boldsymbol{\phi}, \dot{\phi}):=\int_{\Omega(\mathbf{q}, \boldsymbol{\phi})} \rho_{F}\left[D \varphi_{r}(\mathbf{q}, \boldsymbol{\phi})\right]\left(\nabla \varphi_{d}(\mathbf{q}, \boldsymbol{\phi}, \dot{\boldsymbol{\phi}})\right)^{T} \mathrm{~d} \mathbf{x},
$$

we rewrite (3.10):

$$
\mathcal{K}_{F}=\frac{1}{2} \dot{\mathbf{q}} \cdot\left[K_{F}^{r}(\mathbf{q}, \boldsymbol{\phi})\right] \dot{\mathbf{q}}+\mathbf{K}_{F}^{r, d}(\mathbf{q}, \phi, \dot{\phi}) \cdot \dot{\mathbf{q}}+\frac{1}{2} K_{F}^{d}(\mathbf{q}, \phi, \dot{\phi}, \dot{\phi}) .
$$


Remark that both quantities $K_{F}^{d}(\mathbf{q}, \boldsymbol{\phi}, \dot{\boldsymbol{\phi}})$ and $\mathbf{K}_{F}^{r, d}(\mathbf{q}, \boldsymbol{\phi}, \dot{\boldsymbol{\phi}}, \dot{\boldsymbol{\phi}})$ are linear with respect to each entry $\dot{\boldsymbol{\phi}}$. Let now $g$ be the gravitational constant and $G(\mathbf{x}):=-g x_{3}$. By virtue of Archimede's principle, the buoyant force on the $i$-th body is:

$$
\mathbf{F}_{b}^{i}\left(\mathbf{q}^{i}, \boldsymbol{\phi}^{i}\right)=\int_{S^{i}\left(\mathbf{q}^{i}, \phi^{i}\right)}\left(\rho_{S}(\mathbf{x})-\rho_{F}\right) \nabla G(\mathbf{x}) \mathrm{d} \mathbf{x}
$$

and the associated potential energy of the system reads:

$$
\mathcal{P}(\mathbf{q}, \boldsymbol{\phi})=-\int_{S(\mathbf{q}, \boldsymbol{\phi})}\left(\rho_{S}(\mathbf{x})-\rho_{F}\right) G(\mathbf{x}) \mathrm{d} \mathbf{x} .
$$

Finally the Lagrangian of the system fluid-bodies is:

$$
\begin{aligned}
\mathcal{L}(\mathbf{q}, \phi, \dot{\mathbf{q}}, \dot{\boldsymbol{\phi}})=\frac{1}{2} \dot{\mathbf{q}} \cdot\left(\left[K_{S}^{r}(\mathbf{q}, \boldsymbol{\phi})\right]+\left[K_{F}^{r}(\mathbf{q}, \boldsymbol{\phi})\right]\right) \dot{\mathbf{q}}+\mathbf{K}_{F}^{r, d}(\mathbf{q}, \boldsymbol{\phi}, \dot{\boldsymbol{\phi}}) \cdot \dot{\mathbf{q}} \\
+\frac{1}{2} K_{S}^{d}(\dot{\boldsymbol{\phi}}, \dot{\boldsymbol{\phi}})+\frac{1}{2} K_{F}^{d}(\mathbf{q}, \boldsymbol{\phi}, \dot{\boldsymbol{\phi}}, \dot{\boldsymbol{\phi}})-\mathcal{P}(\mathbf{q}, \boldsymbol{\phi}) .
\end{aligned}
$$

In order to study the regularity with respect to $(\mathbf{q}, \boldsymbol{\phi})$ of the quantities $(3.11)$, we apply results of shape sensitivity analysis.

\section{Shape Sensitivity analysis}

This section is self-contained and address an abstract shape optimization problem.

4.1. The context. Let $\mathbb{B}$ be a large ball in $\mathbb{R}^{3}$ centered at the origin and $\Omega$ be an open connected set in $\mathbb{R}^{3}$ which may be bounded or not. We assume that $\partial \Omega=\Gamma_{1} \cup \Gamma_{2}$ such that $\Gamma_{1} \cap \Gamma_{2}=\emptyset, \Gamma_{2} \subset \mathbb{B}$ is compact and $\Gamma_{1}$ is either a hyperplane, either bounded, either the empty set. For any $\phi \in C_{0}^{l}\left(\mathbb{B}, \mathbb{R}^{3}\right),(m \geq 1)$, we set $\boldsymbol{\Phi}:=\left[\mathbb{I}_{3}\right]+\phi$. In all the sequel, we assume that $\|\phi\|_{1, \infty}<1$ so that the function $\boldsymbol{\Phi}$ is a $C^{m}$-diffeomorphism from $\mathbb{R}^{3}$ onto $\mathbb{R}^{3}$. We assume also that $\operatorname{supp} \phi \cap \Gamma_{1}=\emptyset$ and we denote $\Omega(\phi):=\boldsymbol{\Phi}(\Omega)$ and $\Gamma_{2}(\phi):=\boldsymbol{\Phi}\left(\Gamma_{2}\right)$. Let $E$ be a Banach space endowed with the norm $\|\cdot\|_{E}$. We consider for any two given functions $(\mathbf{x}, \boldsymbol{\psi}) \in \Gamma_{2}(\boldsymbol{\phi}) \times E \mapsto b_{i}(\boldsymbol{\phi}, \boldsymbol{\psi}, \mathbf{x}) \in \mathbb{R}, i=1,2$ the functions $u_{1}(\boldsymbol{\phi}, \boldsymbol{\psi}, \cdot)$ and $u_{2}(\phi, \psi, \cdot)$ that solve the following Neumann boundary value problem:

$$
\begin{gathered}
-\Delta u_{i}(\boldsymbol{\phi}, \boldsymbol{\psi}, \cdot)=0 \text { in } \Omega(\boldsymbol{\phi}), \\
\partial_{\mathbf{n}} u_{i}(\boldsymbol{\phi}, \boldsymbol{\psi}, \cdot)=0 \text { on } \Gamma_{1}, \quad \partial_{\mathbf{n}} u_{i}(\boldsymbol{\phi}, \boldsymbol{\psi}, \cdot)=b_{i}(\boldsymbol{\phi}, \boldsymbol{\psi}, \cdot) \text { on } \Gamma_{2}(\boldsymbol{\phi}),
\end{gathered}
$$

for $i=1,2$, the vector $\mathbf{n}$ being the unitary normal to $\partial \Omega(\phi)$ directed toward the exterior of $\Omega(\phi)$. If $\Omega$ is bounded, we assume furthermore that the compatibility conditions:

$$
\int_{\Gamma_{2}(\phi)} b_{i}(\boldsymbol{\phi}, \boldsymbol{\psi}, \mathbf{x}) \mathrm{d} \Gamma_{x}=0, \quad i=1,2
$$

are satisfied. These conditions are not needed any longer when $\Omega$ is not bounded, as it is explained in Ref. [5]. The aim of this section is to study the regularity with respect to $(\boldsymbol{\phi}, \boldsymbol{\psi})$ in a neighborhood of $\left\{\left[\mathbb{O}_{3}\right]\right\} \times E \subset C_{0}^{m}\left(\mathbb{B}, \mathbb{R}^{3}\right) \times E$ of the functional:

$$
J(\boldsymbol{\phi}, \boldsymbol{\psi}):=\int_{\Omega(\boldsymbol{\phi})} \nabla u_{1}(\boldsymbol{\phi}, \boldsymbol{\psi}, \mathbf{x}) \cdot \nabla u_{2}(\boldsymbol{\phi}, \boldsymbol{\psi}, \mathbf{x}) \mathrm{d} \mathbf{x} .
$$


4.2. Some function spaces. In order to study the existence and uniqueness of solutions for the Neumann boundary value problem (4.1), we introduce the following weighted or quotient Lebesgue and Sobolev spaces. We denote $\mathcal{D}^{\prime}(\Omega(\phi))$ the distribution space and $L^{2}(\Omega(\phi)), H^{1}(\Omega(\phi)), H^{2}(\Omega(\phi))$ and $H^{1 / 2}\left(\Gamma_{2}(\phi)\right)$ stand for the classic Lebesgue and Sobolev spaces.

The case $\Omega$ bounded. We define the quotient spaces:

- $L_{N}^{2}(\Omega(\phi)):=L^{2}(\Omega(\phi)) / \mathbb{R}$. The quotient means that we identify two functions which differ from an additive constant. This space is endowed with the scalar product $(u, v)_{L_{N}^{2}}:=\int_{\Omega(\phi)} u v \mathrm{~d} \mathbf{x}-\left(\int_{\Omega(\phi)} u \mathrm{~d} \mathbf{x}\right)\left(\int_{\Omega(\phi)} v \mathrm{~d} \mathbf{x}\right)$.

- $H_{N}^{1}(\Omega(\phi)):=H^{1}(\Omega(\phi)) / \mathbb{R}$ endowed with the scalar product $(u, v)_{H_{N}^{1}}:=$ $\int_{\Omega(\phi)} \nabla u \cdot \nabla v \mathrm{~d} \mathbf{x}$.

- $H_{N}^{2}(\Omega(\phi)):=H^{2}(\Omega(\phi)) / \mathbb{R}$ endowed with the scalar product $(u, v)_{H_{N}^{2}}:=$ $(u, v)_{H_{N}^{1}}+\int_{\Omega(\phi)} D^{2} u: D^{2} v \mathrm{~d} \mathbf{x}$, where $D^{2} u$ and $D^{2} v$ are the Hessian matrices of $u$ and $v$.

Assuming that $\Gamma_{2}$ is Lipschitz continuous (and then also $\Gamma_{2}(\phi)$ since $\phi$ is at least continuously differentiable), we define:

$$
L_{N}^{2}\left(\Gamma_{2}(\phi)\right):=\left\{u \in L^{2}\left(\Gamma_{2}(\phi)\right): \int_{\Gamma_{2}(\phi)} u(\mathbf{x}) \mathrm{d} \Gamma_{x}=0\right\}
$$

and if $\Gamma_{2}$ is of class $C^{1,1}$ and $\phi$ twice continuously differentiable, $H_{N}^{1 / 2}\left(\Gamma_{2}(\phi)\right):=$ $H^{1 / 2}\left(\Gamma_{2}(\phi)\right) \cap L_{N}^{2}\left(\Gamma_{2}(\phi)\right)$.

The case $\Omega$ not bounded. The definitions of the functions spaces turn into:

- $L_{N}^{2}(\Omega(\phi)):=\left\{u \in \mathcal{D}^{\prime}(\Omega(\phi)): \sqrt{1+|\mathbf{x}|^{2}} u \in L^{2}(\Omega(\phi))\right\}$ endowed with the scalar product $(u, v)_{L_{N}^{2}}:=\int_{\Omega(\phi)} u v\left(1+|\mathbf{x}|^{2}\right) \mathrm{d} \mathbf{x}$.

- $H_{N}^{1}(\Omega(\phi)):=\left\{u \in \mathcal{D}^{\prime}(\Omega(\phi)): u / \sqrt{1+|\mathbf{x}|^{2}} \in L^{2}(\Omega(\phi)), \partial_{x_{i}} u \in L^{2}(\Omega(\phi)), i=\right.$ $1,2,3\}$ endowed with the scalar product $(u, v)_{H_{N}^{1}}:=\int_{\Omega(\phi)} u v /\left(1+|\mathbf{x}|^{2}\right) \mathrm{d} \mathbf{x}+$ $\int_{\Omega(\phi)} \nabla u \cdot \nabla v \mathrm{~d} \mathbf{x}$.

- $H_{N}^{2}(\Omega(\phi)):=\left\{u \in H_{N}^{1}(\Omega(\phi)): \sqrt{1+|\mathbf{x}|^{2}} \partial_{x_{i} x_{j}}^{2} u \in L_{N}^{2}(\Omega(\phi)), i, j=\right.$ $1,2,3\}$ endowed with the scalar product $(u, v)_{H_{N}^{2}}:=(u, v)_{H_{N}^{1}}+\int_{\Omega(\phi)} D^{2} u$ : $D^{2} v\left(1+|\mathbf{x}|^{2}\right) \mathrm{d} \mathbf{x}$.

Assuming that $\Gamma_{2}$ is Lipschitz continuous, we set $L_{N}^{2}\left(\Gamma_{2}(\phi)\right):=L^{2}\left(\Gamma_{2}(\phi)\right)$ and if $\Gamma_{2}$ is of class $C^{1,1}$ and $\phi$ twice continuously differentiable, $H_{N}^{1 / 2}\left(\Gamma_{2}(\phi)\right):=$ $H^{1 / 2}\left(\Gamma_{2}(\phi)\right)$.

\subsection{Regularity results.}

Proposition 1 (Existence and uniqueness). Assume that $\partial \Omega$ is Lipschitz continuous. Then for any $(\boldsymbol{\phi}, \boldsymbol{\psi}) \in C_{0}^{1}\left(\mathbb{B}, \mathbb{R}^{3}\right) \times E$ and for any $b_{i}(\boldsymbol{\phi}, \boldsymbol{\psi}) \in L_{N}^{2}\left(\Gamma_{2}(\boldsymbol{\phi})\right)$, the system (4.1) admits a unique weak solution $u_{i}(\boldsymbol{\phi}, \boldsymbol{\psi}) \in H_{N}^{1}(\Omega(\boldsymbol{\phi}))$ defined by:

$$
\int_{\Omega(\phi)} \nabla u_{i}(\boldsymbol{\phi}, \boldsymbol{\psi}, \mathbf{x}) \cdot \nabla \varphi(\mathbf{x}) \mathrm{d} \mathbf{x}=\int_{\Gamma_{2}(\phi)} b_{i}(\phi, \psi, \mathbf{x}) \varphi(\mathbf{x}) \mathrm{d} \Gamma_{x}, \quad \forall \varphi \in H_{N}^{1}(\Omega(\phi)) .
$$

Moreover, there exists a constant $C_{1}(\Omega(\phi))>0$ such that

$$
\left\|u_{i}(\boldsymbol{\phi}, \boldsymbol{\psi})\right\|_{H_{N}^{1}} \leq C_{1}(\Omega(\boldsymbol{\phi}))\left\|b_{i}(\boldsymbol{\phi}, \boldsymbol{\psi})\right\|_{L_{N}^{2}} .
$$


If $\partial \Omega$ is of class $C^{1,1}, \phi \in C_{0}^{2}\left(\mathbb{B}, \mathbb{R}^{3}\right)$ and $b_{i}(\boldsymbol{\phi}, \boldsymbol{\psi}) \in H_{N}^{1 / 2}\left(\Gamma_{2}(\boldsymbol{\phi})\right)$, the solution $u_{i}(\boldsymbol{\phi}, \boldsymbol{\psi})$ is in $H_{N}^{2}(\Omega(\phi))$ and there exists a constant $C_{2}(\Omega(\phi))>0$ such that

$$
\left\|u_{i}(\boldsymbol{\phi}, \boldsymbol{\psi})\right\|_{H_{N}^{2}} \leq C_{2}(\Omega(\boldsymbol{\phi}))\left\|b_{i}(\boldsymbol{\phi}, \boldsymbol{\psi})\right\|_{H_{N}^{1 / 2}} .
$$

These results are classic when $\Omega$ is bounded. For the others cases and the use of weighted Sobolev spaces, we refer to Ref. [2], [1] and [5].

From now on, we will denote $U_{i}(\boldsymbol{\phi}, \boldsymbol{\psi}, \cdot):=u_{i}(\boldsymbol{\phi}, \boldsymbol{\psi}, \boldsymbol{\Phi}(\cdot)), B_{i}(\boldsymbol{\phi}, \boldsymbol{\psi}, \cdot):=b_{i}(\boldsymbol{\phi}, \boldsymbol{\psi}, \boldsymbol{\Phi}(\cdot))$ and $\mathcal{B}_{i}(\phi, \psi, \cdot):=B_{i}(\phi, \psi, \cdot) \operatorname{Jac}_{\Gamma}(\phi)$, $\operatorname{Jac}_{\Gamma}(\phi)$ being the tangential Jacobian defined by $\operatorname{Jac}_{\Gamma}(\phi):=\left|[D \boldsymbol{\Phi}]^{-1 \top} \mathbf{n} \| \operatorname{det}[D \boldsymbol{\Phi}]\right|$. Notice that if $\partial \Omega$ is Lipschitz continuous, $\boldsymbol{\phi} \in C_{0}^{1}\left(\mathbb{B}, \mathbb{R}^{3}\right)$ and $b_{i}(\boldsymbol{\phi}, \boldsymbol{\psi}) \in L_{N}^{2}\left(\Gamma_{2}(\boldsymbol{\phi})\right)$ then $B_{i}(\boldsymbol{\phi}, \boldsymbol{\psi}) \in L^{2}\left(\Gamma_{2}\right)$ and

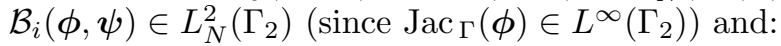

$$
\int_{\Gamma_{2}(\phi)} b_{i}(\boldsymbol{\phi}, \boldsymbol{\psi}, \mathbf{x}) \mathrm{d} \Gamma_{x}=\int_{\Gamma_{2}} \mathcal{B}_{i}(\boldsymbol{\phi}, \boldsymbol{\psi}, \mathbf{x}) \mathrm{d} \Gamma_{x}
$$

In the same way, if $\partial \Omega$ is of class $C^{1,1}, \phi \in C_{0}^{2}\left(\mathbb{B}, \mathbb{R}^{3}\right)$ and $b_{i}(\phi, \psi) \in H_{N}^{1 / 2}\left(\Gamma_{2}(\phi)\right)$ then $B_{i}(\boldsymbol{\phi}, \boldsymbol{\psi}) \in H^{1 / 2}\left(\Gamma_{2}\right)$ and $\mathcal{B}_{i}(\boldsymbol{\phi}, \boldsymbol{\psi}) \in H_{N}^{1 / 2}\left(\Gamma_{2}\right)$.

Proposition 2 (Regularity of the solutions). Assume that $\partial \Omega$ is Lipschitz continuous and that the given mapping $(\boldsymbol{\phi}, \boldsymbol{\psi}) \in C_{0}^{1}\left(\mathbb{B}, \mathbb{R}^{3}\right) \times E \mapsto B_{i}(\boldsymbol{\phi}, \boldsymbol{\psi}) \in L^{2}\left(\Gamma_{2}\right)$ is of class $C^{k}(1 \leq k \leq \infty)$ (respectively analytic) and satisfies $b_{i}(\boldsymbol{\phi}, \boldsymbol{\psi}) \in L_{N}^{2}\left(\Gamma_{2}(\boldsymbol{\phi})\right)$ for all $(\phi, \psi) \in C_{0}^{1}\left(\mathbb{B}, \mathbb{R}^{3}\right) \times E$. Then both mappings:

$$
\begin{aligned}
& (\boldsymbol{\phi}, \boldsymbol{\psi}) \in C_{0}^{1}\left(\mathbb{B}, \mathbb{R}^{3}\right) \times E \mapsto U_{i}(\boldsymbol{\phi}, \boldsymbol{\psi}) \in H_{N}^{1}(\Omega), \\
& (\boldsymbol{\phi}, \boldsymbol{\psi}) \in C_{0}^{1}\left(\mathbb{B}, \mathbb{R}^{3}\right) \times E \mapsto J(\boldsymbol{\phi}, \boldsymbol{\psi}) \in \mathbb{R},
\end{aligned}
$$

are well defined and also of class $C^{k}$ (respectively analytic) in a neighborhood of $\left\{\left[\mathbb{O}_{3}\right]\right\} \times E \in C_{0}^{1}\left(\mathbb{B}, \mathbb{R}^{3}\right) \times E$.

If $\partial \Omega$ is of class $C^{1,1}$ and the mapping $(\boldsymbol{\phi}, \boldsymbol{\psi}) \in C_{0}^{2}\left(\mathbb{B}, \mathbb{R}^{3}\right) \times E \mapsto B_{i}(\boldsymbol{\phi}, \boldsymbol{\psi}) \in$ $H^{1 / 2}\left(\Gamma_{2}\right)$ is of class $C^{1}$ and satisfies $b_{i}(\boldsymbol{\phi}, \boldsymbol{\psi}) \in H_{N}^{1 / 2}\left(\Gamma_{2}(\boldsymbol{\phi})\right)$ for all $(\boldsymbol{\phi}, \boldsymbol{\psi}) \in$ $C_{0}^{2}\left(\mathbb{B}, \mathbb{R}^{3}\right) \times E$, then the mapping:

$$
(\boldsymbol{\phi}, \boldsymbol{\psi}) \in C_{0}^{2}\left(\mathbb{B}, \mathbb{R}^{3}\right) \times E \mapsto U_{k}(\boldsymbol{\phi}, \boldsymbol{\psi}) \in H_{N}^{2}(\Omega),
$$

is well defined and also of class $C^{1}$ in a neighborhood of $\left\{\left[\mathbb{O}_{3}\right]\right\} \times E \subset C_{0}^{2}\left(\mathbb{B}, \mathbb{R}^{3}\right) \times E$. Furthermore, for all compact set $K \in \Omega$ we have $K \subset \Omega(\phi)$ whenever $\|\phi\|_{1, \infty}$ remains small enough and the mappings:

$$
\begin{aligned}
& (\boldsymbol{\phi}, \boldsymbol{\psi}) \in C_{0}^{1}\left(\mathbb{B}, \mathbb{R}^{3}\right) \times\left. E \mapsto u_{i}(\boldsymbol{\phi}, \boldsymbol{\psi})\right|_{K} \in L^{2}(K), \\
& (\boldsymbol{\phi}, \boldsymbol{\psi}) \in C_{0}^{2}\left(\mathbb{B}, \mathbb{R}^{3}\right) \times\left. E \mapsto \partial_{x_{j}} u_{i}(\boldsymbol{\phi}, \boldsymbol{\psi})\right|_{K} \in L^{2}(K), \quad i=1,2, \quad j=1,2,3,
\end{aligned}
$$

are well defined and of class $C^{1}$ in a neighborhood of $\left\{\left[\mathbb{O}_{3}\right]\right\} \times E$. Moreover, we have the following regularity for the partial derivatives:

$$
\begin{aligned}
\left\langle\partial_{\boldsymbol{\phi}} u_{i}\left(\left[\mathbb{O}_{3}\right], \boldsymbol{\psi}, \cdot\right), \boldsymbol{\xi}\right\rangle & \in H_{N}^{1}(\Omega), \quad \forall \boldsymbol{\psi} \in E, \forall \boldsymbol{\xi} \in C_{0}^{1}\left(\mathbb{B}, \mathbb{R}^{3}\right), \\
\left\langle\partial_{\boldsymbol{\phi}}\left(\partial_{x_{j}} u_{i}\right)\left(\left[\mathbb{O}_{3}\right], \boldsymbol{\psi}, \cdot\right), \boldsymbol{\xi}\right\rangle & \in L^{2}(\Omega), \quad \forall \boldsymbol{\psi} \in E, \forall \boldsymbol{\xi} \in C_{0}^{2}\left(\mathbb{B}, \mathbb{R}^{3}\right), j=1,2,3 .
\end{aligned}
$$

For any given $C^{1}$-functions $t \in \mathbb{R} \mapsto \boldsymbol{\psi}(t) \in E$ and $t \in \mathbb{R} \mapsto \boldsymbol{\phi}(t, \cdot) \in C_{0}^{2}\left(\mathbb{B}, \mathbb{R}^{3}\right)$ such that $\phi(0, \cdot)=\left[\mathbb{O}_{3}\right]$ and $\|\phi(t)\|_{1, \infty}<1$ for all $t \in \mathbb{R}$, we denote $\boldsymbol{\Phi}(t):=$ $\left[\mathbb{I}_{3}\right]+\boldsymbol{\phi}(t), \Omega(t):=\boldsymbol{\Phi}(t, \Omega), u_{i}(t):=u_{i}(\boldsymbol{\phi}(t), \boldsymbol{\psi}(t)), b_{i}(t):=b_{i}(\boldsymbol{\phi}(t), \boldsymbol{\psi}(t)), B_{i}(t):=$ $B_{i}(\boldsymbol{\phi}(t), \boldsymbol{\psi}(t))$ for $i=1,2$ and

$$
J(t):=\int_{\Omega(t)} \nabla u_{1}(t) \cdot \nabla u_{2}(t) \mathrm{d} \mathbf{x} .
$$


We can compute the expression of $\partial_{t} J(t)$ :

Proposition 3. Assume that $\partial \Omega$ is of class $C^{1,1}$ and $t \mapsto B_{i}(t) \in H^{1 / 2}\left(\Gamma_{2}\right)$ is of class $C^{1}$. Then, denoting $\mathbf{v}(t, \mathbf{x}):=\partial_{t} \phi\left(t, \boldsymbol{\Phi}^{-1}(t, \mathbf{x})\right)$ for all $\mathbf{x} \in \mathbb{B}$ and $\mathbf{v}_{\Gamma}(t, \mathbf{x}):=$ $\mathbf{v}(t, \mathbf{x})-(\mathbf{v}(t, \mathbf{x}) \cdot \mathbf{n}) \mathbf{n}$ for all $\mathbf{x} \in \Gamma_{2}(t)$, we have:

$$
\begin{aligned}
\partial_{t} J(t)= & -\int_{\Gamma_{2}(t)} \nabla u_{1}(t) \cdot \nabla u_{2}(t)(\mathbf{v}(t) \cdot \mathbf{n}) \mathrm{d} \Gamma_{x} \\
& +\int_{\Gamma_{2}(t)}\left(\partial_{t} B_{1}\left(\mathbf{\Phi}^{-1}(t)\right)-\nabla_{\Gamma} b_{1}(t) \cdot \mathbf{v}_{\Gamma}(t)\right) u_{2}(t) \mathrm{d} \Gamma_{x} \\
& +\int_{\Gamma_{2}(t)}\left(\partial_{t} B_{2}\left(\Phi^{-1}(t)\right)-\nabla_{\Gamma} b_{2}(t) \cdot \mathbf{v}_{\Gamma}(t)\right) u_{1}(t) \mathrm{d} \Gamma_{x} \\
& +2 \int_{\Gamma_{2}(t)} b_{1}(t) b_{2}(t)(\mathbf{v}(t) \cdot \mathbf{n}) \mathrm{d} \Gamma_{x} \\
& -2 \int_{\Gamma_{2}(t)} \mathcal{H}(t)\left(b_{1}(t) u_{2}(t)+b_{2}(t) u_{1}(t)\right)(\mathbf{v}(t) \cdot \mathbf{n}) \mathrm{d} \Gamma_{x}
\end{aligned}
$$

where $\nabla_{\Gamma}$ is the tangential gradient, $\kappa_{1}(t, \mathbf{x})$ and $\kappa_{2}(t, \mathbf{x})$ stand for the principal curvatures of the surface $\Gamma_{2}(t)$ at the point $\mathbf{x}$ and $\mathcal{H}(t, \mathbf{x}):=\left(\kappa_{1}(t, \mathbf{x})+\kappa_{2}(t, \mathbf{x})\right) / 2$ is the mean curvature.

The proofs of Propositions 2 and 3 are similar to the ones of Theorem 5.5.1 page 203 in Ref. [18]. It is written out thoroughly in Ref. [31].

\section{EXISTENCE, UNIQUENESS AND REGULARITY OF THE SWIMMERS TRAJECTORIES}

This section is devoted to the proofs of Theorem 1 and Corollary 1. Notations are those of Section 3.

5.1. The problem in local coordinates. We assume in all this subsection that the assumptions of Theorem 1 are satisfied, namely $\partial \Omega$ is Lipschitz continuous and $m=1$ in (2.8).

It is clear that $(\mathbf{q}, \phi) \in \mathfrak{Q}_{a l}^{n, 1} \mapsto\left[K_{S}^{r}(\mathbf{q}, \phi)\right] \in \mathcal{M}(6 n)$ defined by (3.7) is analytic. In the same way, $\left(\boldsymbol{\psi}_{1}, \boldsymbol{\psi}_{2}\right) \in C_{0}^{1}\left(\mathbb{B}, \mathbb{R}^{3}\right)^{2} \mapsto K_{S}^{d}\left(\boldsymbol{\psi}_{1}, \boldsymbol{\psi}_{2}\right) \in \mathbb{R}$ defined by (3.8) is bilinear, continuous and hence analytic.

To study the regularity of $(\mathbf{q}, \phi) \in \mathfrak{Q}_{a l}^{n, 1} \mapsto \mathcal{P}(\mathbf{q}, \phi) \in \mathbb{R}$, we rewrite it as follows:

$$
\mathcal{P}(\mathbf{q}, \boldsymbol{\phi})=-\int_{S_{0}} \rho_{S}^{0} G(\boldsymbol{\Lambda}(\mathbf{q}, \boldsymbol{\phi}, \mathbf{x})) \mathrm{d} \mathbf{x}+\int_{S_{0}} \rho_{F} G(\boldsymbol{\Lambda}(\mathbf{q}, \boldsymbol{\phi}, \mathbf{x})) \operatorname{det}|[D \boldsymbol{\Lambda}(\mathbf{q}, \boldsymbol{\phi}, \mathbf{x})]| \mathrm{d} \mathbf{x},
$$

in which $\boldsymbol{\Lambda}(\mathbf{q}, \boldsymbol{\phi})$ is given in (3.2). Since we have:

$$
\begin{aligned}
& \int_{S_{0}} \rho_{F} G(\boldsymbol{\Lambda}(\mathbf{q}, \boldsymbol{\phi}, \mathbf{x})) \operatorname{det}|[D \boldsymbol{\Lambda}(\mathbf{q}, \boldsymbol{\phi}, \mathbf{x})]| \mathrm{d} \mathbf{x}= \\
& \sum_{i=1}^{n} \int_{S_{0}^{i}} \rho_{F} G(\boldsymbol{\Lambda}(\mathbf{q}, \boldsymbol{\phi}, \mathbf{x})) \operatorname{det}\left|\left[D \boldsymbol{\Phi}^{i}\left(\mathbf{x}-\mathbf{h}_{0}^{i}\right)\right]\right| \mathrm{d} \mathbf{x},
\end{aligned}
$$

we conclude that $(\mathbf{q}, \phi) \in \mathfrak{Q}_{a l}^{n, 1} \mapsto \mathcal{P}(\mathbf{q}, \phi) \in \mathbb{R}$ is analytic. 
The Stokes formula yields, for all $(\mathbf{q}, \boldsymbol{\phi}) \in \mathfrak{Q}_{a l}^{n, 1}$ and all $i=1, \ldots, n$ :

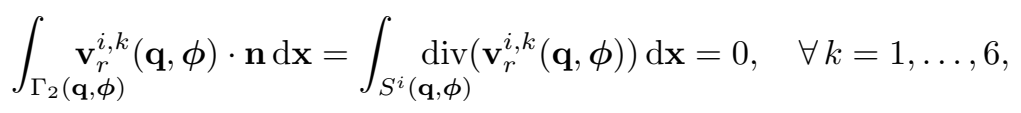

the vector field $\mathbf{v}_{r}^{i, k}(\mathbf{q}, \boldsymbol{\phi})$ being defined by (3.4). The hypothesis (2.7) ensures that, for all $(\mathbf{q}, \phi, \dot{\mathbf{q}}, \dot{\phi}) \in T \mathfrak{Q}_{a l}^{n, 1}$ and all $i=1, \ldots, n$ :

$$
\sum_{i=1}^{n} \int_{\Gamma_{2}(\mathbf{q}, \boldsymbol{\phi})} \mathbf{v}_{d}^{i}(\mathbf{q}, \boldsymbol{\phi}, \dot{\phi}) \cdot \mathbf{n}=0
$$

where $\mathbf{v}_{d}^{i}(\mathbf{q}, \boldsymbol{\phi}, \dot{\boldsymbol{\phi}})$ is given in (3.3). Hence Proposition 1 applies to the functions $\varphi_{r}^{i, k}$ and $\varphi_{d}$ defined by (3.9). We deduce that $\left[K_{F}^{r}(\mathbf{q}, \boldsymbol{\phi})\right], K_{F}^{d}(\mathbf{q}, \boldsymbol{\phi}, \dot{\boldsymbol{\phi}}, \dot{\boldsymbol{\phi}})$ and $\mathbf{K}_{F}^{r, d}(\mathbf{q}, \boldsymbol{\phi}, \dot{\boldsymbol{\phi}})$ given in (3.11) are well defined for all $(\mathbf{q}, \phi, \dot{\mathbf{q}}, \dot{\boldsymbol{\phi}}) \in T \mathfrak{Q}_{a l}^{n, 1}$. We address now the sensitivity of $K_{F}^{d}(\mathbf{q}, \boldsymbol{\phi}, \dot{\boldsymbol{\phi}}, \dot{\boldsymbol{\phi}})$ and $\mathbf{K}_{F}^{r, d}(\mathbf{q}, \boldsymbol{\phi}, \dot{\boldsymbol{\phi}})$ with respect to $(\mathbf{q}, \boldsymbol{\phi}, \dot{\mathbf{q}}, \dot{\boldsymbol{\phi}})$. To match notations of Proposition 2, we define:

$$
\begin{aligned}
b_{r}^{i, k}(\mathbf{q}, \boldsymbol{\phi}, \mathbf{x}):=\mathbf{v}_{r}^{i, k}(\mathbf{q}, \boldsymbol{\phi}, \mathbf{x}) \cdot \mathbf{n}(\mathbf{x}), & \mathbf{x} \in \Gamma_{2}(\mathbf{q}, \boldsymbol{\phi}), \forall k=1, \ldots, 6, \\
b_{d}^{i}(\mathbf{q}, \boldsymbol{\phi}, \dot{\boldsymbol{\phi}}, \mathbf{x}):=\mathbf{v}_{d}^{i}(\mathbf{q}, \boldsymbol{\phi}, \dot{\boldsymbol{\phi}}, \mathbf{x}) \cdot \mathbf{n}(\mathbf{x}), & \mathbf{x} \in \Gamma_{2}(\mathbf{q}, \boldsymbol{\phi}), \forall i=1, \ldots, n,
\end{aligned}
$$

and $B_{r}^{i, k}(\mathbf{q}, \boldsymbol{\phi}, \mathbf{x}):=b_{r}^{i, k}(\mathbf{q}, \boldsymbol{\phi}, \boldsymbol{\Lambda}(\mathbf{q}, \boldsymbol{\phi}, \mathbf{x}))$ and $B_{d}^{i}(\mathbf{q}, \boldsymbol{\phi}, \dot{\boldsymbol{\phi}}, \mathbf{x}):=b_{d}^{i}(\mathbf{q}, \boldsymbol{\phi}, \dot{\boldsymbol{\phi}}, \boldsymbol{\Lambda}(\mathbf{q}, \boldsymbol{\phi}, \mathbf{x}))$ for all $\mathbf{x} \in \Gamma_{2}$. We easily obtain that, for all $\mathbf{x} \in \Gamma_{2}(\mathbf{q}, \phi)$ and all $i=1, \ldots, n$ :

$$
\begin{aligned}
& B_{r}^{i, k}(\mathbf{q}, \boldsymbol{\phi}, \mathbf{x})= \\
& \left\{\begin{array}{lll}
\left\{\mathcal{R}\left(\boldsymbol{\alpha}^{i}\right)^{T} \boldsymbol{\omega}^{i}\right) \wedge \boldsymbol{\Phi}^{i}\left(\mathbf{x}-\mathbf{h}_{0}^{i}\right) \cdot \mathbf{n}\left(\boldsymbol{\Phi}\left(\mathbf{x}-\mathbf{h}_{0}^{i}\right)\right) & \text { if } k=1,2,3, \\
\mathcal{R}\left(\boldsymbol{\alpha}^{i}\right)^{T} \mathbf{e}_{k-3} \cdot \mathbf{n}\left(\boldsymbol{\Phi}\left(\mathbf{x}-\mathbf{h}_{0}^{i}\right)\right) & \text { if } k=4,5,6, & \text { if } \mathbf{x} \in \Gamma_{2}^{i}, \\
0 & & \text { otherwise, }
\end{array}\right.
\end{aligned}
$$

and

$$
B_{d}^{i}(\mathbf{q}, \boldsymbol{\phi}, \dot{\boldsymbol{\phi}}, \mathbf{x})= \begin{cases}\dot{\phi}\left(\mathbf{x}-\mathbf{h}_{0}^{i}\right) \cdot \mathbf{n}\left(\mathbf{\Phi}^{i}\left(\mathbf{x}-\mathbf{h}_{0}^{i}\right)\right) & \text { if } \mathbf{x} \in \Gamma_{2}^{i}, \\ 0 & \text { otherwise }\end{cases}
$$

At this point we need the following proposition whose proof in given in Ref. [31]:

Proposition 4. Let $\Gamma$ be a Lipschitz continuous surface in $\mathbb{R}^{3}$ and denote:

$$
C_{i n v}^{m, \infty}\left(\mathbb{R}^{3}, \mathbb{R}^{3}\right):=\left\{\boldsymbol{\Phi} \in C^{m, \infty}\left(\mathbb{R}^{3}, \mathbb{R}^{3}\right):\left\|\boldsymbol{\Phi}-\left[\mathbb{I}_{3}\right]\right\|_{1, \infty}<1\right\}, \quad(0 \leq m \leq \infty),
$$

(see B). Then:

(1) It is possible to define $\mathbf{x}$-almost everywhere on $\Gamma$ a unitary normal vector $\mathbf{n}(\mathbf{x})$.

(2) The normal vector $\mathbf{n}(\mathbf{x})$ being given $\mathbf{x}-$ a. e. on $\Gamma$, for all $\mathbf{\Phi} \in C_{\text {inv }}^{1, \infty}\left(\mathbb{R}^{3}, \mathbb{R}^{3}\right)$ such that $\left\|\mathbf{\Phi}-\left[\mathbb{I}_{3}\right]\right\|_{1, \infty}<1$, it is possible to define $\mathbf{x}$-almost everywhere on $\boldsymbol{\Phi}(\Gamma)$ a unitary normal vector $\mathbf{n}(\boldsymbol{\Phi}, \mathbf{x})$ such that $\mathbf{n}\left(\left[\mathbb{I}_{3}\right], \mathbf{x}\right)=\mathbf{n}(\mathbf{x})$ for almost every $\mathbf{x} \in \Gamma$ and such that the mapping $\mathbf{\Phi} \in C_{\text {inv }}^{1, \infty}\left(\mathbb{R}^{3}, \mathbb{R}^{3}\right) \mapsto \mathbf{n}(\boldsymbol{\Phi}, \boldsymbol{\Phi}(\cdot)) \in$ $L^{\infty}(\Gamma)$ be analytic. We denote $\mathbf{N}(\mathbf{\Phi}, \mathbf{x}):=\mathbf{n}(\mathbf{\Phi}, \mathbf{\Phi}(\mathbf{x}))$.

(3) There exists $\mathbf{x}-$ a.e. on $\boldsymbol{\Phi}(\Gamma)$ a tangent plane. Denoting $\left\{\boldsymbol{\tau}_{1}(\boldsymbol{\Phi}), \boldsymbol{\tau}_{2}(\boldsymbol{\Phi})\right\}$ an orthogonal basis of this plane such that $\left\{\mathbf{n}(\boldsymbol{\Phi}), \boldsymbol{\tau}_{1}(\boldsymbol{\Phi}), \boldsymbol{\tau}_{2}(\boldsymbol{\Phi})\right\}$ be a direct orthogonal basis of $\mathbb{R}^{3}$, we have $\mathbf{x}-a$. e. on $\boldsymbol{\Phi}(\Gamma)$, for all $\boldsymbol{\xi} \in C^{1, \infty}\left(\mathbb{R}^{3}, \mathbb{R}^{3}\right)$ :

$$
\left\langle\partial_{\boldsymbol{\Phi}} \mathbf{N}\left(\boldsymbol{\Phi}, \boldsymbol{\Phi}^{-1}\right), \boldsymbol{\xi}\right\rangle=-\sum_{i=1}^{2} \mathbf{n}(\boldsymbol{\Phi}) \cdot\left[D\left(\boldsymbol{\xi} \circ \boldsymbol{\Phi}^{-1}\right)\right] \boldsymbol{\tau}_{i}(\boldsymbol{\Phi}) .
$$


Let us consider a given $C^{k}$-function, $1 \leq k \leq \infty$ (respectively analytic) $t \in$ $[0, \infty) \mapsto \boldsymbol{\Phi}(t) \in C_{\text {inv }}^{1, \infty}\left(\mathbb{R}^{3}, \mathbb{R}^{3}\right)$ such that $\mathbf{\Phi}(0)=\left[\mathbb{I}_{3}\right]$ and denote $\mathbf{n}(t):=\mathbf{n}(\boldsymbol{\Phi}(t))$, $\boldsymbol{\tau}_{k}(t)=\boldsymbol{\tau}_{k}(\boldsymbol{\Phi}(t)), \mathbf{N}(t):=\mathbf{N}(\boldsymbol{\Phi}(t)), \mathbf{v}(t, \mathbf{x}):=\partial_{t} \boldsymbol{\Phi}\left(t, \boldsymbol{\Phi}^{-1}(t, \mathbf{x})\right)$ and $\mathbf{v}_{\Gamma}(\mathbf{x}, t):=$ $\mathbf{v}(t, \mathbf{x})-(\mathbf{v}(t, \mathbf{x}) \cdot \mathbf{n}(t, \mathbf{x})) \mathbf{n}(t, \mathbf{x})$ (the tangential part of $\mathbf{v}(t, \mathbf{x}))$. Then, the identity (5.1) reads:

$$
\partial_{t} \mathbf{N}\left(t, \Phi^{-1}(t)\right)=-\sum_{k=1}^{2}\left(\mathbf{n}(t) \cdot[D \mathbf{v}(t)] \boldsymbol{\tau}_{k}(t)\right) \boldsymbol{\tau}_{k}(t),
$$

almost everywhere on $\boldsymbol{\Phi}(t, \Gamma)$.

Assume now that $\Gamma$ is $C^{1,1}$ and $\mathbf{\Phi} \in C_{\text {inv }}^{2, \infty}\left(\mathbb{R}^{3}, \mathbb{R}^{3}\right)$. At any point $\mathbf{x}$ of $\boldsymbol{\Phi}(t, \Gamma)$, $\Pi_{2}(t, \mathbf{x})$ stands for the second fundamental form. Then the mapping: $t \in[0, \infty) \mapsto$ $\mathbf{N}(t) \in L^{\infty}(\Gamma)$ is of class $C^{k}$ (respectively analytic) in a neighborhood of $t=0$ and for all $\mathbf{x} \in \mathbf{\Phi}(t, \Gamma)$ :

$$
\partial_{t} \mathbf{N}\left(t, \Phi^{-1}(t, \mathbf{x})\right)=-\nabla_{\Gamma}(\mathbf{v}(t, \mathbf{x}) \cdot \mathbf{n}(t, \mathbf{x}))-\left\langle\Pi_{2}(t, \mathbf{x}), \mathbf{v}_{\Gamma}(t, \mathbf{x}), \mathbf{v}_{\Gamma}(t, \mathbf{x})\right\rangle
$$

where $\nabla_{\Gamma}$ is the tangential gradient. Introducing also $\kappa_{1}(t, \mathbf{x})$ and $\kappa_{2}(t, \mathbf{x})$ the principal curvatures of $\boldsymbol{\Phi}\left(t, \Gamma_{2}\right)$ at the point $\mathbf{x}$ and choosing for $\boldsymbol{\tau}_{1}(t, \mathbf{x}), \boldsymbol{\tau}_{2}(t, \mathbf{x})$ the associated principal directions such that $\left\langle\Pi_{2}(t, \mathbf{x}), \boldsymbol{\tau}_{i}(t, \mathbf{x}), \boldsymbol{\tau}_{i}(t, \mathbf{x})\right\rangle=-\left([D \mathbf{n}(t, \mathbf{x})] \boldsymbol{\tau}_{i}(t, \mathbf{x})\right)$. $\boldsymbol{\tau}_{i}(t, \mathbf{x})=\kappa_{i}(t, \mathbf{x}), i=1,2$, we also have:

$$
\left\langle\Pi_{2}(t, \mathbf{x}), \mathbf{v}_{\Gamma}(t, \mathbf{x}), \mathbf{v}_{\Gamma}(t, \mathbf{x})\right\rangle=\sum_{i=1}^{2} \kappa_{i}(t, \mathbf{x})\left(\mathbf{v}(t, \mathbf{x}) \cdot \boldsymbol{\tau}_{i}(t, \mathbf{x})\right) \boldsymbol{\tau}_{i}(t, \mathbf{x}) .
$$

Using this proposition, we deduce that the functions $(\mathbf{q}, \phi) \in \mathfrak{Q}_{a l}^{n, 1} \mapsto B_{r}^{i, k}(\mathbf{q}, \phi) \in$ $L^{2}\left(\Gamma_{2}\right)$ and $(\mathbf{q}, \phi, \dot{\mathbf{q}}, \dot{\boldsymbol{\phi}}) \in T \mathfrak{Q}_{a l}^{n, 1} \mapsto B_{d}^{i}(\mathbf{q}, \boldsymbol{\phi}, \dot{\boldsymbol{\phi}}) \in L^{2}\left(\Gamma_{2}\right)$ are analytic. We conclude, applying Proposition 2, that all the quantities defined by (3.11) have the same regularity and hence that $\mathcal{L}$ is analytic.

Computing each term of (2.10) from the expression (3.13), we obtain that (2.10) reads:

$$
\begin{aligned}
&\left\langle\left[K_{S}^{r}(\mathbf{q}, \boldsymbol{\phi})\right]\right.\left.+\left[K_{F}^{r}(\mathbf{q}, \boldsymbol{\phi})\right], \ddot{\mathbf{q}}, \cdot\right\rangle \\
&+\left\langle\partial_{\mathbf{q}}\left[K^{r}(\mathbf{q}, \boldsymbol{\phi})\right], \dot{\mathbf{q}}, \dot{\mathbf{q}}, \cdot\right\rangle-\frac{1}{2}\left\langle\partial_{\mathbf{q}}\left[K^{r}(\mathbf{q}, \boldsymbol{\phi})\right], \cdot \dot{\mathbf{q}}, \dot{\mathbf{q}}\right\rangle \\
&+\left\langle\partial_{\phi}\left[K_{S}^{r}(\mathbf{q}, \boldsymbol{\phi})\right], \dot{\boldsymbol{\phi}}, \dot{\mathbf{q}}, \cdot\right\rangle+\left\langle\partial_{\boldsymbol{\phi}}\left[K_{F}^{r}(\mathbf{q}, \boldsymbol{\phi})\right], \dot{\boldsymbol{\phi}}, \dot{\mathbf{q}}, \cdot\right\rangle \\
&+\left\langle\partial_{\mathbf{q}} \mathbf{K}_{F}^{r, d}(\mathbf{q}, \boldsymbol{\phi}), \dot{\mathbf{q}}, \dot{\boldsymbol{\phi}}, \cdot\right\rangle-\left\langle\partial_{\mathbf{q}} \mathbf{K}_{F}^{r, d}(\mathbf{q}, \boldsymbol{\phi}), \cdot, \dot{\boldsymbol{\phi}}, \dot{\mathbf{q}}\right\rangle \\
&+\left\langle\mathbf{K}_{F}^{r, d}(\mathbf{q}, \boldsymbol{\phi}), \ddot{\boldsymbol{\phi}}, \cdot\right\rangle+\left\langle\partial_{\phi} \mathbf{K}_{F}^{r, d}(\mathbf{q}, \boldsymbol{\phi}), \dot{\boldsymbol{\phi}}, \dot{\boldsymbol{\phi}}, \cdot\right\rangle-\frac{1}{2}\left\langle\partial_{\mathbf{q}} K_{F}^{d}(\mathbf{q}, \boldsymbol{\phi}), \cdot, \dot{\boldsymbol{\phi}}, \dot{\boldsymbol{\phi}}\right\rangle \\
&+\left\langle\partial_{\mathbf{q}} \mathcal{P}(\mathbf{q}, \boldsymbol{\phi}), \cdot\right\rangle=\mathbf{0}_{6 n},
\end{aligned}
$$

where $\left[K^{r}(\mathbf{q}, \phi)\right]=\left[K_{S}^{r}(\mathbf{q}, \phi)\right]+\left[K_{F}^{r}(\mathbf{q}, \phi)\right]$. Let us make precise the nature of each term arising in this expression:

- $\left[K_{S}^{r}(\mathbf{q}, \phi)\right]+\left[K_{F}^{r}(\mathbf{q}, \phi)\right]$ is a $6 n \times 6 n$ symmetric matrix, that is to say an element of $L_{2}\left(\mathbb{R}^{6 n} \times \mathbb{R}^{6 n}, \mathbb{R}\right)$,

- $\partial_{\mathbf{q}}\left[K_{S}^{r}(\mathbf{q}, \boldsymbol{\phi})\right]$ and $\partial_{\mathbf{q}}\left[K_{F}^{r}(\mathbf{q}, \phi)\right]$ are $6 n \times 6 n \times 6 n$ third-rank tensors, that is to say elements of $L_{3}\left(\mathbb{R}^{6 n} \times \mathbb{R}^{6 n} \times \mathbb{R}^{6 n}, \mathbb{R}\right)$,

- $\partial_{\boldsymbol{\phi}}\left[K_{S}^{r}(\mathbf{q}, \boldsymbol{\phi})\right]$ and $\partial_{\boldsymbol{\phi}}\left[K_{F}^{r}(\mathbf{q}, \boldsymbol{\phi})\right]$ lies in $L_{3}\left(C_{0}^{1}\left(\mathbb{B}, \mathbb{R}^{3}\right)^{n} \times \mathbb{R}^{6 n} \times \mathbb{R}^{6 n}, \mathbb{R}\right)$,

- $\partial_{\mathbf{q}} \mathbf{K}_{F}^{r, d}(\mathbf{q}, \boldsymbol{\phi})$ is an element of $L_{3}\left(\mathbb{R}^{6 n} \times C_{0}^{1}\left(\mathbb{B}, \mathbb{R}^{3}\right)^{n} \times \mathbb{R}^{6 n}, \mathbb{R}\right)$,

- $\mathbf{K}_{F}^{r, d}(\mathbf{q}, \boldsymbol{\phi})$ belongs to $L_{2}\left(C_{0}^{1}\left(\mathbb{B}, \mathbb{R}^{3}\right)^{n} \times \mathbb{R}^{6 n}, \mathbb{R}\right)$, 
- $\partial_{\mathbf{q}} K_{F}^{d}(\mathbf{q}, \phi)$ is in $L_{3}\left(\mathbb{R}^{6 n} \times C_{0}^{1}\left(\mathbb{B}, \mathbb{R}^{3}\right)^{n} \times C_{0}^{1}\left(\mathbb{B}, \mathbb{R}^{3}\right)^{n}, \mathbb{R}\right)$,

- $\partial_{\phi} \mathbf{K}_{F}^{r, d}(\mathbf{q}, \boldsymbol{\phi})$ is in $L_{3}\left(C_{0}^{1}\left(\mathbb{B}, \mathbb{R}^{3}\right)^{n} \times C_{0}^{1}\left(\mathbb{B}, \mathbb{R}^{3}\right)^{n} \times \mathbb{R}^{6 n}, \mathbb{R}\right)$,

- $\partial_{\mathbf{q}} \mathcal{P}(\mathbf{q}, \boldsymbol{\phi})$ is a $6 n$-length column vector, that is an element of $L_{1}\left(\mathbb{R}^{6 n}, \mathbb{R}\right)$.

The last remaining point to prove before applying the Cauchy-Lipschitz Theorem to the ODE (5.4) is:

Lemma 1. For all $(\mathbf{q}, \boldsymbol{\phi}) \in \mathfrak{Q}_{a l}^{n, 1}$, let $\lambda^{i}\left(\phi^{i}\right)>0, i=1, \ldots, n$ be the smallest eigenvalue of the inertia tensor $\left[I_{0}^{i}\left(\phi^{i}\right)\right]$ defined by (3.6) and remind that $m^{i}>0$ is the mass of the $i-$ th body. Then, for all $\dot{\mathbf{q}} \in \mathbb{R}^{6 n}$ :

$$
\dot{\mathbf{q}} \cdot\left(\left[K_{S}^{r}(\mathbf{q}, \phi)\right]+\left[K_{F}^{r}(\mathbf{q}, \phi)\right]\right) \dot{\mathbf{q}} \geq \min _{1 \leq i \leq n}\left\{\lambda^{i}\left(\phi^{i}\right) \cos ^{2}\left(\alpha_{2}^{i}\right), m^{i} \cos ^{2}\left(\alpha_{2}^{i}\right)\right\}|\dot{\mathbf{q}}|^{2}
$$

In particular, the family of symmetric matrices $\left[K_{S}^{r}(\mathbf{q}, \boldsymbol{\phi})\right]+\left[K_{F}^{r}(\mathbf{q}, \boldsymbol{\phi})\right]$ is uniformly elliptic on $\mathbb{R}^{6 n} \times \mathbb{R}^{6 n}$ whenever $(\mathbf{q}, \boldsymbol{\phi})$ remains in a compact subset of $\mathfrak{Q}_{a l}^{n, 1}$.

Proof : Beforehand, notice that since $\dot{\mathbf{q}} \cdot\left[K_{F}^{r}(\mathbf{q}, \phi)\right] \dot{\mathbf{q}}:=\int_{\Omega(\mathbf{q}, \phi)}\left|\nabla\left(\boldsymbol{\varphi}_{r} \cdot \dot{\mathbf{q}}\right)\right|^{2} \mathrm{~d} \mathbf{x}$, the matrix $\left[M_{F}^{r}(\mathbf{q}, \phi)\right]$ is positive definite for any $(\mathbf{q}, \phi) \in \mathfrak{Q}_{a l}^{n, 1}$. The estimate arises, taking into account the definition $(3.7)$ of $\left[K_{S}^{r}(\mathbf{q}, \boldsymbol{\phi})\right]$ and remarking that, for all $i=1, \ldots, n$ :

$$
\min _{\dot{\mathbf{q}}^{i} \neq \mathbf{0}_{6}} \frac{\dot{\mathbf{q}}^{i} \cdot\left[\omega^{i}\right]^{T} \mathcal{R}\left(\boldsymbol{\alpha}^{i}\right)\left[I_{0}^{i}\left(\boldsymbol{\phi}^{i}\right)\right] \mathcal{R}\left(\boldsymbol{\alpha}^{i}\right)^{T}\left[\omega^{i}\right] \dot{\mathbf{q}}^{i}}{\left|\dot{\mathbf{q}}^{i}\right|^{2}}=\min _{\dot{\mathbf{q}}^{i} \neq \mathbf{0}_{6}} \frac{\dot{\mathbf{q}}^{i} \cdot\left[I_{0}^{i}\left(\boldsymbol{\phi}^{i}\right)\right] \dot{\mathbf{q}}^{i}}{\left|\left[\omega^{i}\right]^{-1} \mathcal{R}\left(\boldsymbol{\alpha}^{i}\right) \dot{\mathbf{q}}^{i}\right|^{2}},
$$

and that $\left|\left[\omega^{i}\right]^{-1} \mathcal{R}\left(\boldsymbol{\alpha}^{i}\right) \dot{\mathbf{q}}^{i}\right| \leq 1 / \cos \left(\alpha_{2}^{i}\right)\left|\dot{\mathbf{q}}^{i}\right|$.

Let now $t \in[0, \infty) \mapsto \phi(t) \in C_{0}^{1}\left(\mathbb{B}, \mathbb{R}^{3}\right)^{n}$ be a given function satisfying the assumptions of Theorem 1 and plug the expression of $\phi(t), \dot{\phi}(t)$ and $\ddot{\phi}(t)$ into the ODE (5.4). Applying the Cauchy-Lipschitz Theorem, we obtain the conclusion of Theorem 1. The proof is then complete.

5.2. The problem in global coordinates. In this section, we tackle the proof of Corollary 1. Under the assumptions of Theorem 1, we denote $\left[K^{r}(\mathbf{q}, \phi)\right]:=$ $\left[K_{S}^{r}(\mathbf{q}, \phi)\right]+\left[K_{F}^{r}(\mathbf{q}, \phi)\right]$ and for all $(\mathbf{q}, \phi, \dot{\mathbf{q}}, \dot{\phi}) \in T \mathfrak{Q}_{a l}^{n, 1}$ :

$$
\begin{aligned}
E(\mathbf{q}, \dot{\mathbf{q}}, \phi) & :=\frac{1}{2} \dot{\mathbf{q}} \cdot\left[K^{r}(\mathbf{q}, \boldsymbol{\phi})\right] \dot{\mathbf{q}}, \\
\left\langle\delta_{1}(\mathbf{q}, \phi), \dot{\boldsymbol{\phi}}\right\rangle & :=\sup _{\dot{\mathbf{q}} \neq \mathbf{0}_{6 n}}-\frac{\left\langle\partial_{\phi}\left[K^{r}(\mathbf{q}, \boldsymbol{\phi})\right], \dot{\boldsymbol{\phi}}, \dot{\mathbf{q}}, \dot{\mathbf{q}}\right\rangle}{\left\langle\left[K^{r}(\mathbf{q}, \boldsymbol{\phi})\right], \dot{\mathbf{q}}, \dot{\mathbf{q}}\right\rangle}, \\
\delta_{2}(\mathbf{q}, \phi, \dot{\boldsymbol{\phi}}, \ddot{\boldsymbol{\phi}}) & :=\sup _{\dot{\mathbf{q}} \neq \mathbf{0}_{6 n}}-\sqrt{2} \frac{\left\langle\mathbf{K}_{F}^{r, d}(\mathbf{q}, \boldsymbol{\phi}), \ddot{\boldsymbol{\phi}}, \dot{\mathbf{q}}\right\rangle+\left\langle\partial_{\phi} \mathbf{K}_{F}^{r, d}(\mathbf{q}, \boldsymbol{\phi}), \dot{\boldsymbol{\phi}}, \dot{\boldsymbol{\phi}}, \dot{\mathbf{q}}\right\rangle}{\sqrt{\left\langle\left[K^{r}(\mathbf{q}, \boldsymbol{\phi})\right], \dot{\mathbf{q}}, \dot{\mathbf{q}}\right\rangle}} .
\end{aligned}
$$


For any $C^{2}$-function $t \in[0, \infty) \mapsto(\mathbf{q}(t), \boldsymbol{\phi}(t)) \in \mathfrak{Q}_{a l}^{n, 1} \operatorname{such}$ that $(\mathbf{q}(t), \boldsymbol{\phi}(t), \dot{\mathbf{q}}(t), \dot{\boldsymbol{\phi}}(t)) \in$ $T \mathfrak{Q}_{a l}^{n, 1}$ we define:

$$
\begin{aligned}
& a(\mathbf{q}, \boldsymbol{\phi}, \dot{\boldsymbol{\phi}}, \ddot{\boldsymbol{\phi}}, t):=\frac{1}{2}\left\langle K_{F}^{d}(\mathbf{q}, \boldsymbol{\phi}), \dot{\boldsymbol{\phi}}, \dot{\boldsymbol{\phi}}\right\rangle-\mathcal{P}(\mathbf{q}, \boldsymbol{\phi}) \\
&+e^{\int_{0}^{t}\left\langle\delta_{1}(\mathbf{q}, \boldsymbol{\phi}), \dot{\boldsymbol{\phi}}\right\rangle d \tau}\left(-\frac{1}{2}\left\langle K_{F}^{d}\left(\mathbf{q}_{0}, \boldsymbol{\phi}_{0}\right), \dot{\boldsymbol{\phi}}_{0}, \dot{\boldsymbol{\phi}}_{0}\right\rangle+\mathcal{P}\left(\mathbf{q}_{0}, \boldsymbol{\phi}_{0}\right)+E\left(\mathbf{q}_{0}, \dot{\mathbf{q}}_{0}, \boldsymbol{\phi}_{0}\right)\right) \\
&+\int_{0}^{t}\left\langle\delta_{1}(\mathbf{q}, \boldsymbol{\phi}), \dot{\boldsymbol{\phi}}\right\rangle\left(\frac{1}{2}\left\langle K_{F}^{d}(\mathbf{q}, \boldsymbol{\phi}), \dot{\boldsymbol{\phi}}, \dot{\boldsymbol{\phi}}\right\rangle-\mathcal{P}(\mathbf{q}, \boldsymbol{\phi})\right) e^{\int_{s}^{t}\left\langle\delta_{1}(\mathbf{q}, \boldsymbol{\phi}), \dot{\boldsymbol{\phi}}\right\rangle d \tau} \mathrm{d} s \\
&+\int_{0}^{t}\left(-\frac{1}{2}\left\langle\partial_{\phi} K_{F}^{d}(\mathbf{q}, \boldsymbol{\phi}), \dot{\boldsymbol{\phi}}, \dot{\boldsymbol{\phi}}, \dot{\boldsymbol{\phi}}\right\rangle-\left\langle K_{F}^{d}(\mathbf{q}, \boldsymbol{\phi}), \ddot{\boldsymbol{\phi}}, \dot{\boldsymbol{\phi}}\right\rangle\right. \\
&\left.+\left\langle\partial_{\boldsymbol{\phi}} \mathcal{P}(\mathbf{q}, \boldsymbol{\phi}), \dot{\boldsymbol{\phi}}\right\rangle\right) e^{\int_{s}^{t}\left\langle\delta_{1}(\mathbf{q}, \boldsymbol{\phi}), \dot{\boldsymbol{\phi}}\right\rangle d \tau} \mathrm{d} s
\end{aligned}
$$

and:

$$
k(\mathbf{q}, \boldsymbol{\phi}, \dot{\boldsymbol{\phi}}, \ddot{\boldsymbol{\phi}}, t, s):=e^{\int_{s}^{t}\left\langle\delta_{1}(\mathbf{q}, \boldsymbol{\phi}), \dot{\boldsymbol{\phi}}\right\rangle d \tau} \delta_{2}(\mathbf{q}, \boldsymbol{\phi}, \dot{\boldsymbol{\phi}}, \ddot{\boldsymbol{\phi}}),
$$

and we set $a^{+}:=\max \{a, 0\}, k^{+}:=\max \{k, 0\}$. According to Lemma 1 and regularity results proved earlier, we deduce that the quantities (5.5) are (at least) Lipschitz continuous with respect to $(\mathbf{q}, \phi, \dot{\mathbf{q}}, \dot{\phi}, \ddot{\phi}) \in T \mathfrak{Q}_{a l}^{n, 1} \times \mathbb{R}^{6 n}$. We draw the same conclusion for the functions $t \in[0, \infty) \mapsto a(\mathbf{q}, \boldsymbol{\phi}, \dot{\boldsymbol{\phi}}, \ddot{\boldsymbol{\phi}}, t) \in \mathbb{R}$ and $(s, t) \in[0, \infty)^{2} \mapsto k(\mathbf{q}, \boldsymbol{\phi}, \dot{\phi}, \ddot{\phi}, t, s) \in \mathbb{R}\left(\right.$ and then also for $a^{+}$and $\left.b^{+}\right)$.

Lemma 2 (Energy estimate). Let $\mathbf{q}^{*}$ be the solution defined on $[0, T)$, arising in Theorem 1 and $\phi$ be the corresponding deformation function. Then, for all $t \in[0, T)$, the following estimate holds:

$$
\begin{aligned}
& E\left(\mathbf{q}^{*}, \dot{\mathbf{q}}^{*}, \boldsymbol{\phi}\right) \leq a^{+}\left(\mathbf{q}^{*}, \boldsymbol{\phi}, \dot{\boldsymbol{\phi}}, \ddot{\boldsymbol{\phi}}, t\right) \\
& +\frac{1}{4}\left[2\left(\int_{0}^{t} k^{+}\left(\mathbf{q}^{*}, \boldsymbol{\phi}, \dot{\boldsymbol{\phi}}, \ddot{\boldsymbol{\phi}}, t, s\right) \sqrt{a^{+}\left(\mathbf{q}^{*}, \boldsymbol{\phi}, \dot{\boldsymbol{\phi}}, \ddot{\boldsymbol{\phi}}, t\right)} \mathrm{d} s\right)^{1 / 2}\right. \\
& \left.\quad+\int_{0}^{t} k^{+}\left(\mathbf{q}^{*}, \boldsymbol{\phi}, \dot{\boldsymbol{\phi}}, \ddot{\boldsymbol{\phi}}, t, s\right) \mathrm{d} s\right]^{2} .
\end{aligned}
$$

It means in particular that $E\left(\mathbf{q}^{*}, \dot{\mathbf{q}}^{*}, \boldsymbol{\phi}\right)$ is not able to blow up in finite time whenever $\left(\mathbf{q}^{*}, \phi\right)$ remains in a compact subset of $\mathfrak{Q}_{a l}^{n, 1}$.

The proof of this technical lemma is given in Ref. [31].

Let $\mathbf{q}^{*}$ be the solution defined on $[0, T)$ given in Theorem 1 and denote $\widetilde{T}:=$ $\sup \left\{t \in[0, T):\left(\mathbf{q}^{*}(s), \boldsymbol{\phi}(s)\right) \in \widetilde{\mathfrak{Q}}_{a l}^{n, 1}, \forall s=[0, t]\right\}$ and $\mathbf{Q}^{*}(t):=\mathfrak{F}_{1}\left(\mathbf{q}^{*}(t)\right)$ for all $t \in\left[0, \widetilde{T}\right.$ ) (see $\mathrm{C}$ for the definitions of $\widetilde{\mathfrak{Q}}_{a l}^{n, 1}$ and $\mathfrak{F}_{1}$ ). Classic behavior results for solutions of ODE's (see for example Theorem 4.1, page 15 in Ref. [11]) ensure that:

$$
\lim _{t \rightarrow \widetilde{T}}\left(t, \mathbf{q}^{*}(t), \boldsymbol{\phi}(t), \dot{\mathbf{q}}^{*}(t), \dot{\phi}(t)\right) \in \partial\left([0, \infty) \times T \widetilde{\mathfrak{Q}}_{a l}^{n, 1}\right) .
$$

It means that one of the following alternatives happens:

- Either $\widetilde{T}=\infty$,

- Either $\left(\mathbf{q}^{*}(\widetilde{T}), \boldsymbol{\phi}(\widetilde{T}), \dot{\mathbf{q}}^{*}(\widetilde{T}), \dot{\boldsymbol{\phi}}(\widetilde{T})\right) \in \partial T \widetilde{\mathfrak{Q}}_{a l}^{n, 1}$. Only two events are possible: 
- Either $\left(\mathbf{q}^{*}(\widetilde{T}), \boldsymbol{\phi}(\widetilde{T})\right) \in \partial \widetilde{\mathfrak{Q}}_{a l}^{n, 1} \backslash \partial \mathfrak{Q}_{a l}^{n, 1}$. Hence, making use of Lemma 2 together with Lemma 1 , we deduce that $\left|\dot{\mathbf{q}}^{*}(\widetilde{T})\right|<\infty$. The configuration (locations and shapes) for the bodies at time $\widetilde{T}$, that is $S\left(\mathbf{q}^{*}(\widetilde{T}), \phi(\widetilde{T})\right)$, can be chosen as new initial configuration. We denote it $S_{0}$ and we can reapply Theorem 1 with $\left(\dot{\mathbf{q}}_{0}, \dot{\boldsymbol{\phi}}_{0}\right)=\left(\dot{\mathbf{q}}^{*}(\widetilde{T}), \dot{\boldsymbol{\phi}}(\widetilde{T})\right)$. We obtain a new function $\mathbf{q}^{*}$ and the function $\mathbf{Q}^{*}$ can be extended to the right of $\widetilde{T}$ by setting $\mathbf{Q}^{*}(\widetilde{T}+t)=\mathfrak{F}_{1}\left(\mathbf{q}^{*}(t)\right)$.

- Either $\left(\mathbf{q}^{*}(\widetilde{T}), \boldsymbol{\phi}(\widetilde{T})\right) \in \partial \mathfrak{Q}_{a l}^{n, 1} \cap \partial \widetilde{\mathfrak{Q}}_{a l}^{n, 1}$. This possibility means that time $\widetilde{T}$ corresponds to the time of a collision between two bodies or between a body with the fluid's domain boundary. It is the only case where we may have $\left|\dot{\mathbf{q}}^{*}(t)\right| \rightarrow \infty$ as $t \rightarrow \widetilde{T}$.

The proof of Corollary 1 is then complete.

\section{EXPlicit Form of the LAGRANGiAn SYSTEM OF ODE'S IN A PARTICUlaR CASE}

In this section we address the case of a swimmer alone and we assume that the system body-fluid fills a bounded region in $\mathbb{R}^{3}$. We denote $\mathbf{h}(t)$ the coordinates of the center of mass of the body with $\mathbf{h}(0)=\mathbf{0}_{3}$. The deformation is given by a $C^{k}$-function $(k \geq 1): t \in[0, \infty) \mapsto \phi(t) \in C_{0}^{m}\left(\mathbb{B}, \mathbb{R}^{3}\right)$ for which $(\phi(t), \dot{\phi}(t)) \in \mathcal{D}^{1, m}$ for all $t \geq 0$ and $\phi(0)=\left[\mathbb{O}_{3}\right]$. We denote $\boldsymbol{\Phi}(t):=\left[\mathbb{I}_{3}\right]+\phi(t)$ and the deformed swimmer is $S_{d}(t):=\mathbf{\Phi}\left(t, S_{0}\right)$. For any pair $(t, \mathbf{q})$ such that $(\mathbf{q}, \boldsymbol{\phi}(t)) \in \mathfrak{Q}_{a l}^{1, m}$, we denote merely $\boldsymbol{\Lambda}(t, \mathbf{q})$ instead of $\boldsymbol{\Lambda}(\mathbf{q}, \boldsymbol{\phi}(t))$ defined by (3.2). We recall that $\boldsymbol{\Lambda}(t, \mathbf{q})$ is a $C^{m}$-diffeomorphism from $\mathbb{R}^{3}$ onto $\mathbb{R}^{3}$. In particular, we have:

$$
\boldsymbol{\Lambda}((t, \mathbf{q}), \mathbf{x})= \begin{cases}\mathcal{R}(\boldsymbol{\alpha}) \mathbf{\Phi}(t, \mathbf{x})+\mathbf{h} & \text { if } \mathbf{x} \in S_{0} \\ \mathbf{x} & \text { if } \mathbf{x} \in \Gamma_{1}\end{cases}
$$

We set $S(t, \mathbf{q}):=\boldsymbol{\Lambda}\left((t, \mathbf{q}), S_{0}\right), \Omega(t, \mathbf{q}):=\boldsymbol{\Lambda}((t, \mathbf{q}), \Omega)$ and $\Gamma_{2}(t, \mathbf{q}):=\partial S(t, \mathbf{q})$. We define also $\widetilde{\boldsymbol{\Lambda}}$, a $C^{m}$-diffeomorphism from $\mathbb{R}^{3}$ onto $\mathbb{R}^{3}$ by $\widetilde{\boldsymbol{\Lambda}}((t, \mathbf{q}), \widetilde{\mathbf{x}}):=$ $\mathcal{R}(\boldsymbol{\alpha})^{T}(\boldsymbol{\Lambda}((t, \mathbf{q}), \widetilde{\mathbf{x}})-\mathbf{h})$ for all $\widetilde{\mathbf{x}} \in \mathbb{R}^{3}$ and we set $\widetilde{S}(t):=\widetilde{\boldsymbol{\Lambda}}\left((t, \mathbf{q}), S_{0}\right)=\mathbf{\Phi}\left(t, S_{0}\right)$, $\widetilde{\Omega}(t, \mathbf{q}):=\widetilde{\boldsymbol{\Lambda}}((t, \mathbf{q}), \Omega), \widetilde{\Gamma}_{2}(t):=\partial \widetilde{S}(t)$ and $\widetilde{\Gamma}_{1}(\mathbf{q}):=\widetilde{\boldsymbol{\Lambda}}\left((t, \mathbf{q}), \Gamma_{1}\right)=\mathcal{R}(\boldsymbol{\alpha})^{T}\left(\Gamma_{1}-\mathbf{h}\right)$. We introduce the following vector fields compactly supported in $\mathbb{R}^{3}$ :

$$
\begin{aligned}
& \mathbf{v}_{k}^{r}((t, \mathbf{q}), \cdot):=\partial_{q_{k}} \mathbf{\Lambda}\left((t, \mathbf{q}), \boldsymbol{\Lambda}^{-1}((t, \mathbf{q}), \cdot)\right), k=1, \ldots, 6, \\
& \mathbf{v}^{d}((t, \mathbf{q}), \cdot):=\partial_{t} \mathbf{\Lambda}\left((t, \mathbf{q}), \boldsymbol{\Lambda}^{-1}((t, \mathbf{q}), \cdot)\right), \\
& \dot{\mathbf{v}}^{d}((t, \mathbf{q}), \cdot):=\partial_{t t}^{2} \mathbf{\Lambda}\left((t, \mathbf{q}), \boldsymbol{\Lambda}^{-1}((t, \mathbf{q}), \cdot)\right), \\
& \widetilde{\mathbf{v}}_{k}^{r}((t, \mathbf{q}), \cdot):=\partial_{q_{k}} \widetilde{\boldsymbol{\Lambda}}\left((t, \mathbf{q}), \widetilde{\boldsymbol{\Lambda}}^{-1}((t, \mathbf{q}), \cdot)\right), k=1, \ldots, 6, \\
& \widetilde{\mathbf{v}}^{d}((t, \mathbf{q}), \cdot):=\partial_{t} \widetilde{\boldsymbol{\Lambda}}\left((t, \mathbf{q}), \widetilde{\boldsymbol{\Lambda}}^{-1}((t, \mathbf{q}), \cdot)\right),
\end{aligned}
$$


and we can make them explicit on the boundaries of $\Omega(t, \mathbf{q})$ and $\widetilde{\Omega}(t, \mathbf{q})$. Thus, we have:

$$
\begin{aligned}
\mathbf{v}_{k}^{r}((t, \mathbf{q}), \mathbf{x}) & =\mathbf{0}_{3} & & \text { on } \Gamma_{1}, k=1, \ldots, 6, \\
\mathbf{v}^{d}((t, \mathbf{q}), \mathbf{x}) & =\mathbf{0}_{3} & & \text { on } \Gamma_{1}, \\
\mathbf{v}_{k}^{r}((t, \mathbf{q}), \mathbf{x}) & =\boldsymbol{\omega}_{k} \wedge(\mathbf{x}-\mathbf{h}) & & \text { on } \Gamma_{2}(t, \mathbf{q}), k=1,2,3, \\
\mathbf{v}_{k}^{r}((t, \mathbf{q}), \mathbf{x}) & =\mathbf{e}_{k-3} & & \text { on } \Gamma_{2}(t, \mathbf{q}), k=4,5,6, \\
\mathbf{v}^{d}((t, \mathbf{q}), \mathbf{x}) & =\mathcal{R}(\boldsymbol{\alpha}) \dot{\boldsymbol{\phi}}\left(\mathbf{p}, \boldsymbol{\Lambda}^{-1}((t, \mathbf{q}), \mathbf{x})\right) & & \text { on } \Gamma_{2}(t, \mathbf{q}), \\
\dot{\mathbf{v}}^{d}((t, \mathbf{q}), \mathbf{x}) & =\mathcal{R}(\boldsymbol{\alpha}) \ddot{\boldsymbol{\phi}}\left(\mathbf{p}, \boldsymbol{\Lambda}^{-1}((t, \mathbf{q}), \mathbf{x})\right) & & \text { on } \Gamma_{2}(t, \mathbf{q}),
\end{aligned}
$$

and:

$$
\begin{array}{ll}
\widetilde{\mathbf{v}}_{k}^{r}((t, \mathbf{q}), \widetilde{\mathbf{x}})=-\left(\mathcal{R}(\boldsymbol{\alpha})^{T} \boldsymbol{\omega}_{k}\right) \wedge \widetilde{\mathbf{x}}, & \text { on } \widetilde{\Gamma}_{1}(\mathbf{q}), k=1,2,3, \\
\widetilde{\mathbf{v}}_{k}^{r}((t, \mathbf{q}), \widetilde{\mathbf{x}})=-\mathcal{R}(\boldsymbol{\alpha})^{T} \mathbf{e}_{k-3} & \text { on } \widetilde{\Gamma}_{1}(\mathbf{q}), k=4,5,6, \\
\widetilde{\mathbf{v}}^{d}((t, \mathbf{q}), \widetilde{\mathbf{x}})=\mathbf{0}_{3} & \text { on } \widetilde{\Gamma}_{1}(\mathbf{q}), \\
\widetilde{\mathbf{v}}_{k}^{r}((t, \mathbf{q}), \widetilde{\mathbf{x}})=\mathbf{0}_{3} & \text { on } \widetilde{\Gamma}_{2}(t), k=1, \ldots, 6, \\
\widetilde{\mathbf{v}}^{d}((t, \mathbf{q}), \widetilde{\mathbf{x}})=\dot{\boldsymbol{\phi}}\left(\mathbf{p}, \widetilde{\boldsymbol{\Lambda}}^{-1}((t, \mathbf{q}), \widetilde{\mathbf{x}})\right) & \text { on } \widetilde{\Gamma}_{2}(t), \\
\dot{\overrightarrow{\mathbf{v}}}^{d}((t, \mathbf{q}), \widetilde{\mathbf{x}})=\ddot{\boldsymbol{\phi}}\left(\mathbf{p}, \widetilde{\boldsymbol{\Lambda}}^{-1}((t, \mathbf{q}), \widetilde{\mathbf{x}})\right) & \text { on } \widetilde{\Gamma}_{2}(t) .
\end{array}
$$

We denote $\left[\widetilde{V}^{r}(t, \mathbf{q})\right]$ the $3 \times 6$ matrix defined on $\mathbb{R}^{3}$ and whose column vectors are $\widetilde{\mathbf{v}}_{k}^{r}, k=1, \ldots, 6$. Like in Section 3 , the potential of the fluid reads $\varphi(t, \mathbf{q})=$ $\sum_{k=1}^{6} \varphi_{k}^{r}(t, \mathbf{q}) \dot{q}_{k}+\varphi^{d}(t, \mathbf{q})$, where $\varphi_{k}^{r}(t, \mathbf{q})$ and $\varphi^{d}(t, \mathbf{q})$ are harmonic in $\Omega(t, \mathbf{q})$ and satisfy the following Neumann boundary conditions:

$$
\begin{aligned}
& \partial_{\mathbf{n}} \varphi_{k}^{r}(t, \mathbf{q})=b_{k}^{r}(t, \mathbf{q}):=\mathbf{v}_{k}^{r}(t, \mathbf{q}) \cdot \mathbf{n} \text { on } \Gamma_{1} \cup \Gamma_{2}(t, \mathbf{q}), \quad k=1, \ldots, 6, \\
& \partial_{\mathbf{n}} \varphi^{d}(t, \mathbf{q})=b^{d}(t, \mathbf{q}):=\mathbf{v}_{d}(t, \mathbf{q}) \cdot \mathbf{n} \text { on } \Gamma_{1} \cup \Gamma_{2}(t, \mathbf{q}) .
\end{aligned}
$$

We introduce also:

$$
\begin{array}{ll}
\widetilde{\varphi}_{k}^{r}((t, \mathbf{q}), \widetilde{\mathbf{x}}):=\varphi_{k}^{r}((t, \mathbf{q}), \mathcal{R}(\boldsymbol{\alpha}) \widetilde{\mathbf{x}}+\mathbf{h}), \quad k=1, \ldots, 6 \\
\widetilde{\varphi}^{d}((t, \mathbf{q}), \widetilde{\mathbf{x}}):=\varphi^{d}((t, \mathbf{q}), \mathcal{R}(\boldsymbol{\alpha}) \widetilde{\mathbf{x}}+\mathbf{h}), \quad \forall \widetilde{\mathbf{x}} \in \widetilde{\Omega}(t, \mathbf{q}) .
\end{array}
$$

The functions $\widetilde{\varphi}_{k}^{r}(t, \mathbf{q})$ and $\widetilde{\varphi}^{d}(t, \mathbf{q})$ are also harmonic in $\widetilde{\Omega}(t, \mathbf{q})$ and satisfy the Neumann boundary conditions:

$$
\begin{array}{ll}
\partial_{\mathbf{n}} \widetilde{\varphi}_{k}^{r}(t, \mathbf{q})=\widetilde{b}_{k}^{r}(t, \mathbf{q}):=0 & \text { on } \widetilde{\Gamma}_{1}(\mathbf{q}), k=1, \ldots, 6, \\
\partial_{\mathbf{n}} \widetilde{\varphi}_{k}^{r}(t, \mathbf{q})=\widetilde{b}_{k}^{r}(t, \mathbf{q}):=\left(\mathcal{R}(\boldsymbol{\alpha})^{T} \boldsymbol{\omega}_{k}\right) \wedge \widetilde{\mathbf{x}} \cdot \mathbf{n} & \text { on } \widetilde{\Gamma}_{2}(t), k=1,2,3, \\
\partial_{\mathbf{n}} \widetilde{\varphi}_{k}^{r}(t, \mathbf{q})=\widetilde{b}_{k}^{r}(t, \mathbf{q}):=\mathcal{R}(\boldsymbol{\alpha})^{T} \mathbf{e}_{k-3} \cdot \mathbf{n} & \text { on } \widetilde{\Gamma}_{2}(t), k=4,5,6, \\
\partial_{\mathbf{n}} \widetilde{\varphi}^{d}(t, \mathbf{q})=\widetilde{b}^{d}(t, \mathbf{q}):=\widetilde{\mathbf{v}}_{d}^{k}(t, \mathbf{q}) \cdot \mathbf{n} & \text { on } \widetilde{\Gamma}_{1}(\mathbf{q}) \cup \widetilde{\Gamma}_{2}(t) .
\end{array}
$$

We set $\varphi^{r}:=\left(\varphi_{1}^{r}, \ldots, \varphi_{6}^{r}\right)^{T}$ and $\widetilde{\varphi}^{r}:=\left(\widetilde{\varphi}_{1}^{r}, \ldots, \widetilde{\varphi}_{6}^{r}\right)^{T}$ and we denote merely $\left[K_{S}^{r}(t, \mathbf{q})\right]$, $\left[K_{F}^{r}(t, \mathbf{q})\right], \mathbf{K}_{F}^{r, d}(t, \mathbf{q})$ and $K_{F}^{d}(t, \mathbf{q})$ instead of $\left[K_{S}^{r}(\mathbf{q}, \boldsymbol{\phi}(t))\right],\left[K_{F}^{r}(\mathbf{q}, \boldsymbol{\phi}(t))\right], \mathbf{K}_{F}^{r, d}(\mathbf{q}, \boldsymbol{\phi}(t), \dot{\phi}(t))$ 
and $K_{F}^{d}(\mathbf{q}, \boldsymbol{\phi}(t), \dot{\boldsymbol{\phi}}(t), \dot{\boldsymbol{\phi}}(t))$ defined in (3.11). The ODE (5.4) becomes:

$$
\begin{aligned}
& \left\langle\left[K_{S}^{r}(t, \mathbf{q})\right]+\left[K_{F}^{r}(t, \mathbf{q})\right], \ddot{\mathbf{q}}, \cdot\right\rangle \\
& +\left\langle\partial_{\mathbf{q}}\left[K_{S}^{r}(t, \mathbf{q})\right], \dot{\mathbf{q}}, \dot{\mathbf{q}}, \cdot\right\rangle-\frac{1}{2}\left\langle\partial_{\mathbf{q}}\left[K_{S}^{r}(t, \mathbf{q})\right], \cdot \dot{\mathbf{q}}, \dot{\mathbf{q}}\right\rangle \\
& +\left\langle\partial_{\mathbf{q}}\left[K_{F}^{r}(t, \mathbf{q})\right], \dot{\mathbf{q}}, \dot{\mathbf{q}}, \cdot\right\rangle-\frac{1}{2}\left\langle\partial_{\mathbf{q}}\left[K_{F}^{r}(t, \mathbf{q})\right], \cdot, \dot{\mathbf{q}}, \dot{\mathbf{q}}\right\rangle \\
& \quad+\left\langle\partial_{t}\left[K_{S}^{r}(t, \mathbf{q})\right], \dot{\mathbf{q}}, \cdot\right\rangle+\left\langle\partial_{t}\left[K_{F}^{r}(t, \mathbf{q})\right], \dot{\mathbf{q}}, \cdot\right\rangle \\
& +\left\langle\partial_{\mathbf{q}} \mathbf{K}_{F}^{r, d}(t, \mathbf{q}), \dot{\mathbf{q}}, \cdot\right\rangle-\left\langle\partial_{\mathbf{q}} \mathbf{K}_{F}^{r, d}(t, \mathbf{q}), \cdot, \dot{\mathbf{q}}\right\rangle+\left\langle\partial_{t} \mathbf{K}_{F}^{r, d}(t, \mathbf{q}), \cdot\right\rangle \\
& \quad-\frac{1}{2}\left\langle\partial_{\mathbf{q}} K_{F}^{d}(t, \mathbf{q}), \cdot\right\rangle+\left\langle\partial_{\mathbf{q}} \mathcal{P}(t, \mathbf{q}), \cdot\right\rangle=\mathbf{0}_{6 n} .
\end{aligned}
$$

The expressions of $\left[K_{S}^{r}(t, \mathbf{q})\right]$ and $\left[K_{F}^{r}(t, \mathbf{q})\right]$ (upon an integration by parts) are given in (D.2). Tedious computations involving the formulas (A.1) yields:

$$
\begin{aligned}
\left\langle\partial_{\mathbf{q}}\left[K_{S}^{r}(t, \mathbf{q})\right], \dot{\mathbf{q}}, \dot{\mathbf{q}}, \cdot\right\rangle-\frac{1}{2}\left\langle\partial_{\mathbf{q}}\left[K_{S}^{r}(t, \mathbf{q})\right], \cdot, \dot{\mathbf{q}}, \dot{\mathbf{q}}\right\rangle= & \\
& \left\langle\llbracket T_{s y m}^{S / r}(t, \mathbf{q}) \rrbracket+\llbracket T_{s k e w}^{S / r}(t, \mathbf{q}) \rrbracket, \dot{\mathbf{q}}, \dot{\mathbf{q}}, \cdot\right\rangle,
\end{aligned}
$$

where the $6 \times 6 \times 6$ third-rank tensors $\llbracket T_{s y m}^{S / r}(t, \mathbf{q}) \rrbracket$ and $\llbracket T_{s k e w}^{S / r}(t, \mathbf{q}) \rrbracket$ are given in (D.3). Without difficulty, we obtain also the identity $\partial_{t}\left[K_{S}^{r}(t, \mathbf{q})\right]=\left[M_{s y m}^{S / d}(t, \mathbf{q})\right]$, the $6 \times 6$ symmetric matrix in the right hand side being given in (D.2). Observe now that we have:

$$
\begin{aligned}
K_{F}^{r}(t, \mathbf{q})_{i j} & =\int_{\widetilde{\Omega}(t, \mathbf{q})} \nabla \widetilde{\varphi}_{i}^{r}(t, \mathbf{q}, \widetilde{\mathbf{x}}) \cdot \nabla \widetilde{\varphi}_{j}^{r}(t, \mathbf{q}, \widetilde{\mathbf{x}}) \mathrm{d} \widetilde{\mathbf{x}}, \quad 1 \leq i, j \leq 6, \\
K_{F}^{r, d}(t, \mathbf{q})_{i} & =\int_{\widetilde{\Omega}(t, \mathbf{q}, \widetilde{\mathbf{x}})} \nabla \widetilde{\varphi}^{d}(t, \mathbf{q}, \widetilde{\mathbf{x}}) \cdot \nabla \widetilde{\varphi}_{i}^{r}(t, \mathbf{q}, \widetilde{\mathbf{x}}) \mathrm{d} \widetilde{\mathbf{x}}, \quad i=1, \ldots, 6,
\end{aligned}
$$

and

$$
K_{F}^{d}(t, \mathbf{q})=\int_{\widetilde{\Omega}(t, \mathbf{q}, \widetilde{\mathbf{x}})}\left|\nabla \widetilde{\varphi}^{d}(t, \mathbf{q}, \widetilde{\mathbf{x}})\right|^{2} \mathrm{~d} \widetilde{\mathbf{x}}
$$

Applying formula (4.3), we obtain:

$$
\partial_{\mathbf{q}} K_{F}^{d}(t, \mathbf{q})=-\int_{\widetilde{\Gamma}_{1}(\mathbf{q})} \rho_{F}\left|\nabla \widetilde{\varphi}^{d}\right|^{2}\left[\widetilde{V}^{r}\right]^{T} \mathbf{n} \mathrm{d} \Gamma_{x}
$$

The change of variables $\mathbf{x}:=\mathcal{R}(\boldsymbol{\alpha}) \widetilde{\mathbf{x}}+\mathbf{h}$ in the integral above yields:

$$
\partial_{\mathbf{q}} K_{F}^{d}(t, \mathbf{q})=-\int_{\Gamma_{1}} \rho_{F}\left|\nabla \varphi^{d}\right|^{2}\left[W^{r}\right]^{T} \mathbf{n} \mathrm{d} \Gamma_{x}
$$

where the matrix $\left[W^{r}\right]$ is given by (D.1). In order to apply again the same formula (4.3) to the others terms, we introduce:

$$
\begin{aligned}
& \widetilde{B}_{k}^{r}(t, \mathbf{q}, \mathbf{x}):=\widetilde{b}_{k}^{r}(t, \mathbf{q}, \widetilde{\Lambda}((t, \mathbf{q}), \mathbf{x})) \text { on } \Gamma_{1} \cup \Gamma_{2}, \quad k=1, \ldots, 6, \\
& \widetilde{B}^{d}(t, \mathbf{q}, \mathbf{x}):=\widetilde{b}^{d}(t, \mathbf{q}, \widetilde{\boldsymbol{\Lambda}}((t, \mathbf{q}), \mathbf{x})) \text { on } \Gamma_{1} \cup \Gamma_{2},
\end{aligned}
$$


ON THE SELF-DISPLACEMENT OF DEFORMABLE BODIES IN A POTENTIAL FLUID FLO

and we have:

$$
\begin{aligned}
\widetilde{B}_{k}^{r}(t, \mathbf{q}, \mathbf{x}) & =0 & & \text { on } \Gamma_{1}, k=1, \ldots 6 \\
\widetilde{B}^{d}(t, \mathbf{q}) & =0 & & \text { on } \Gamma_{1}, \\
\widetilde{B}_{k}^{r}(t, \mathbf{q}, \mathbf{x}) & =\left(\mathcal{R}(\boldsymbol{\alpha})^{T} \boldsymbol{\omega}_{k}\right) \wedge \boldsymbol{\Phi}(t, \mathbf{x}) \cdot \mathbf{n}(\mathbf{\Phi}(t, \mathbf{x})) & & \text { on } \Gamma_{2}, k=1,2,3, \\
\widetilde{B}_{k}^{r}(t, \mathbf{q}) & =\mathcal{R}(\boldsymbol{\alpha})^{T} \mathbf{e}_{k-3} \cdot \mathbf{n}(\mathbf{\Phi}(t, \mathbf{x})) & & \text { on } \Gamma_{2}, k=4,5,6, \\
\widetilde{B}^{d}(t, \mathbf{q}) & =\dot{\phi}(t, \mathbf{x}) \cdot \mathbf{n}(\boldsymbol{\Phi}(t)) & & \text { on } \Gamma_{2}, k=1, \ldots, d .
\end{aligned}
$$

Straightforward computations yield for all $\widetilde{\mathbf{x}} \in \widetilde{\Gamma}_{1}(\mathbf{q})$ and for all $k=1, \ldots, 6$, $\partial_{q_{i}} \widetilde{B}_{k}^{r}\left((t, \mathbf{q}), \widetilde{\boldsymbol{\Lambda}}^{-1}((t, \mathbf{q}), \widetilde{\mathbf{x}})\right)=0$ and for all $i=1, \ldots, 6$ and all $\mathbf{x} \in \widetilde{\Gamma}_{2}(t)$ we have:

$\partial_{q_{i}} \widetilde{B}_{k}^{r}\left((t, \mathbf{q}), \widetilde{\boldsymbol{\Lambda}}^{-1}((t, \mathbf{q}), \widetilde{\mathbf{x}})\right)= \begin{cases}\left(\boldsymbol{\omega}_{k} \wedge \boldsymbol{\omega}_{i}\right) \cdot \mathcal{R}(\boldsymbol{\alpha})(\widetilde{\mathbf{x}} \wedge \mathbf{n}) & \text { if } 1 \leq k<i \leq 3, \\ \left(\mathcal{R}(\boldsymbol{\alpha}) \mathbf{n} \wedge \mathbf{e}_{k-3}\right) \cdot \boldsymbol{\omega}_{i} & \text { if } k=4,5,6, i=1,2,3 \\ 0 & \text { otherwise. }\end{cases}$

We denote $[\widetilde{N}(t, \mathbf{q})]$ the $6 \times 6$ matrix whose entries are $\widetilde{N}_{k i}(t, \mathbf{q}):=\partial_{q_{i}} \widetilde{B}_{k}^{r}\left(t, \mathbf{q}, \widetilde{\boldsymbol{\Lambda}}^{-1}(t, \mathbf{q})\right)$ and invoking formula (4.3), we get:

$$
\begin{aligned}
&\left\langle\partial_{\mathbf{q}}\left[K_{F}^{r}(t, \mathbf{q})\right], \dot{\mathbf{q}}, \dot{\mathbf{q}}, \cdot\right\rangle-\frac{1}{2}\left\langle\partial_{\mathbf{q}}\left[K_{F}^{r}(t, \mathbf{q})\right], \cdot \dot{\mathbf{q}}, \dot{\mathbf{q}}\right\rangle= \\
&-\frac{1}{2} \int_{\widetilde{\Gamma}_{1}(\mathbf{q})} \rho_{F}\left[D \widetilde{\varphi}^{r}\right]\left[D \widetilde{\varphi}^{r}\right]^{T} \dot{\mathbf{q}}\left(\mathbf{n} \cdot\left[D \widetilde{V}^{r}\right] \dot{\mathbf{q}}\right) \mathrm{d} \Gamma_{x}+\frac{1}{2} \int_{\widetilde{\Gamma}_{2}(t)} \rho_{F} \widetilde{\varphi}^{r}\left(\dot{\mathbf{q}} \cdot\left([\tilde{N}]+[\tilde{N}]^{T}\right) \dot{\mathbf{q}}\right) \mathrm{d} \Gamma_{x} \\
& \quad+\frac{1}{2} \int_{\widetilde{\Gamma}_{1}(\mathbf{q})} \rho_{F}\left(\left(\dot{\mathbf{q}}^{T}[D \widetilde{\varphi}][D \widetilde{\varphi}]^{T} \dot{\mathbf{q}}\right)\left(\left[\widetilde{V}^{r}\right]^{T} \mathbf{n}\right)-\left[D \widetilde{\varphi}^{r}\right]\left[D \widetilde{\varphi}^{r}\right]^{T} \dot{\mathbf{q}}\left(\mathbf{n} \cdot\left[D \widetilde{V}^{r}\right] \dot{\mathbf{q}}\right)\right) \mathrm{d} \Gamma_{x} \\
&+\int_{\widetilde{\Gamma}_{2}(t)} \rho_{F}\left(\widetilde{\varphi}^{r} \cdot \dot{\mathbf{q}}\right)\left([\tilde{N}]-[\tilde{N}]^{T}\right) \dot{\mathbf{q}} \mathrm{d} \Gamma_{x} .
\end{aligned}
$$

Applying the change of variables $\mathbf{x}:=\mathcal{R}(\boldsymbol{\alpha}) \widetilde{\mathbf{x}}+\mathbf{h}$, we get:

$$
\left\langle\partial_{\mathbf{q}}\left[K_{F}^{r}(t, \mathbf{q})\right], \dot{\mathbf{q}}, \dot{\mathbf{q}}, \cdot\right\rangle-\frac{1}{2}\left\langle\partial_{\mathbf{q}}\left[K_{F}^{r}(t, \mathbf{q})\right], \cdot \dot{\mathbf{q}}, \dot{\mathbf{q}}\right\rangle=\left\langle\llbracket T_{s y m}^{F / r} \rrbracket+\llbracket T_{s k e w}^{F / r} \rrbracket, \dot{\mathbf{q}}, \dot{\mathbf{q}}\right\rangle,
$$

in which the expressions of the $6 \times 6 \times 6$ third-rank tensors $\llbracket T_{s y m}^{F / r} \rrbracket$ and $\llbracket T_{s y m}^{F / r} \rrbracket$ are given by (D.3). The same formula (4.3) yields also:

$$
\begin{aligned}
& \left\langle\partial_{\mathbf{q}} \mathbf{K}_{F}^{r, d}(t, \mathbf{q}), \dot{\mathbf{q}}, \cdot\right\rangle-\left\langle\partial_{\mathbf{q}} \mathbf{K}_{F}^{r, d}(t, \mathbf{q}), \cdot \dot{\mathbf{q}}\right\rangle= \\
& -\int_{\widetilde{\Gamma}_{1}(\mathbf{q})} \rho_{F}\left(\left[D \widetilde{\varphi}^{r}\right]\left(\nabla \widetilde{\varphi}^{d}\right)^{T}\left(\dot{\mathbf{q}} \cdot\left[\widetilde{V}^{r}\right]^{T} \mathbf{n}\right)-\left(\dot{\mathbf{q}} \cdot\left[D \widetilde{\varphi}^{r}\right]\left(\nabla \widetilde{\varphi}^{d}\right)^{T}\right)\left[\widetilde{V}^{r}\right]^{T} \mathbf{n}\right) \mathrm{d} \Gamma_{x} \\
& \quad+\int_{\widetilde{\Gamma}_{2}(t)} \rho_{F} \widetilde{\varphi}^{d}\left([\widetilde{N}]-[\tilde{N}]^{T}\right) \dot{\mathbf{q}} \mathrm{d} \Gamma_{x} .
\end{aligned}
$$

The change of variables $\mathbf{x}:=\mathcal{R}(\boldsymbol{\alpha}) \widetilde{\mathbf{x}}+\mathbf{h}$ leads to:

$$
\left\langle\partial_{\mathbf{q}} \mathbf{K}_{F}^{r, d}(t, \mathbf{q}), \dot{\mathbf{q}}, \cdot\right\rangle-\left\langle\partial_{\mathbf{q}} \mathbf{K}_{F}^{r, d}(t, \mathbf{q}), \cdot \dot{\mathbf{q}}\right\rangle=\left[M_{s k e w}^{F / d}(t, \mathbf{q})\right] \dot{\mathbf{q}}
$$

the definition of $6 \times 6$ skew-symmetric matrix $\left[M_{\text {skew }}^{F / d}(t, \mathbf{q})\right]$ being given in (D.4). As regards the computation of $\partial_{t} \widetilde{B}^{d}(t, \mathbf{q})$, we require the use of Lemma 4 . Since 
$\widetilde{\boldsymbol{\Lambda}}(t, \mathbf{q}, \widetilde{\mathbf{x}})=\mathbf{\Phi}(t, \widetilde{\mathbf{x}})$ on $\widetilde{\Gamma}_{2}(t)$, we obtain that:

$$
\begin{aligned}
& \partial_{t} \widetilde{B}_{k}^{r}\left(t, \mathbf{q}, \widetilde{\boldsymbol{\Lambda}}^{-1}(t, \mathbf{q}, \widetilde{\mathbf{x}})\right)= \\
& \begin{cases}\mathcal{R}(\boldsymbol{\alpha})\left(\widetilde{\mathbf{v}}^{d} \wedge \mathbf{n}+\widetilde{\mathbf{x}} \wedge\left(\left(-\mathbf{n} \cdot\left[D \widetilde{\mathbf{v}}^{d}\right] \boldsymbol{\tau}_{1}\right) \boldsymbol{\tau}_{1}+\left(-\mathbf{n} \cdot\left[D \widetilde{\mathbf{v}}^{d}\right] \boldsymbol{\tau}_{2}\right) \boldsymbol{\tau}_{2}\right)\right) \cdot \boldsymbol{\omega}_{k} & \text { if } k=1,2,3, \\
\mathcal{R}(\boldsymbol{\alpha})\left(\left(-\mathbf{n} \cdot\left[D \widetilde{\mathbf{v}}^{d}\right] \boldsymbol{\tau}_{1}\right) \boldsymbol{\tau}_{1}+\left(-\mathbf{n} \cdot\left[D \widetilde{\mathbf{v}}^{d}\right] \boldsymbol{\tau}_{2}\right) \boldsymbol{\tau}_{2}\right) \cdot \mathbf{e}_{k-3} & \text { if } k=4,5,6 .\end{cases}
\end{aligned}
$$

We also have on $\widetilde{\Gamma}_{2}(t)$ :

$$
\begin{aligned}
& \nabla_{\Gamma} \widetilde{b}_{k}^{r}(t, \mathbf{q}, \widetilde{\mathbf{x}}) \cdot \widetilde{\mathbf{v}}^{d}= \\
& \begin{cases}\mathcal{R}(\boldsymbol{\alpha})\left(\widetilde{\mathbf{v}}^{d} \wedge \mathbf{n}+\widetilde{\mathbf{x}} \wedge\left(-\kappa_{1}\left(\boldsymbol{\tau}_{1} \cdot \widetilde{\mathbf{v}}^{d}\right) \boldsymbol{\tau}_{1}-\kappa_{2}\left(\boldsymbol{\tau}_{2} \cdot \widetilde{\mathbf{v}}^{d}\right) \boldsymbol{\tau}_{2}\right)\right) \cdot \boldsymbol{\omega}_{k} & \text { if } k=1,2,3, \\
\mathcal{R}(\boldsymbol{\alpha})\left(-\kappa_{1}\left(\boldsymbol{\tau}_{1} \cdot \widetilde{\mathbf{v}}^{d}\right) \boldsymbol{\tau}_{1}-\kappa_{2}\left(\boldsymbol{\tau}_{2} \cdot \widetilde{\mathbf{v}}^{d}\right) \boldsymbol{\tau}_{2}\right) \cdot \mathbf{e}_{k-3} & \text { if } k=4,5,6 .\end{cases}
\end{aligned}
$$

But the definition of the tangential gradient allows us to write $\nabla_{\Gamma}\left(\widetilde{\mathbf{v}}^{d} \cdot \mathbf{n}\right)=\left(\nabla\left(\widetilde{\mathbf{v}}^{d}\right.\right.$. $\left.\mathbf{n}) \cdot \boldsymbol{\tau}_{1}\right) \boldsymbol{\tau}_{1}+\left(\nabla\left(\widetilde{\mathbf{v}}^{d} \cdot \mathbf{n}\right) \cdot \boldsymbol{\tau}_{2}\right) \boldsymbol{\tau}_{2}$ and according to the definition of the principal curvature $\kappa_{i}$, we get $\nabla\left(\widetilde{\mathbf{v}}^{d} \cdot \mathbf{n}\right) \cdot \boldsymbol{\tau}_{k}=\mathbf{n} \cdot\left[D \widetilde{\mathbf{v}}^{d}\right] \boldsymbol{\tau}_{k}+\widetilde{\mathbf{v}}^{d} \cdot[D \mathbf{n}] \boldsymbol{\tau}_{k}=\mathbf{n} \cdot\left[D \widetilde{\mathbf{v}}^{d}\right] \boldsymbol{\tau}_{k}-\kappa_{k}\left(\widetilde{\mathbf{v}}^{d} \cdot \boldsymbol{\tau}_{k}\right)$. We deduce that on $\widetilde{\Gamma}_{2}(t)$ :

$$
\begin{aligned}
\partial_{t} \widetilde{B}_{k}^{r}\left(t, \mathbf{q}, \widetilde{\boldsymbol{\Lambda}}^{-1}(t, \mathbf{q}, \widetilde{\mathbf{x}})\right)-\nabla_{\Gamma} \widetilde{b}_{k}^{r}(t, \mathbf{q}, \widetilde{\mathbf{x}}) \cdot \widetilde{\mathbf{v}}^{d}= \\
\qquad \begin{array}{ll}
-\mathcal{R}(\boldsymbol{\alpha})\left(\widetilde{\mathbf{x}} \wedge \nabla_{\Gamma}\left(\widetilde{\mathbf{v}}^{d} \cdot \mathbf{n}\right)\right) \cdot \boldsymbol{\omega}_{k} & \text { if } k=1,2,3, \\
-\mathcal{R}(\boldsymbol{\alpha}) \nabla_{\Gamma}\left(\widetilde{\mathbf{v}}^{d} \cdot \mathbf{n}\right) \cdot \mathbf{e}_{k-3} & \text { if } k=4,5,6 .
\end{array}
\end{aligned}
$$

We denote $\widetilde{\mathbf{A}}(t, \mathbf{q})$ the 6 -length column vector defined on $\widetilde{\Gamma}_{2}(t)$ whose entries are $\widetilde{A}_{k}(t, \mathbf{q}):=\partial_{t} \widetilde{B}_{k}^{r}\left(t, \mathbf{q}, \widetilde{\Lambda}^{-1}(t, \mathbf{q})\right)-\nabla_{\Gamma} \widetilde{b}_{k}^{r}(t, \mathbf{q}) \cdot \widetilde{\mathbf{v}}^{d}$ and applying one more time formula (4.3) we obtain:

$$
\begin{aligned}
& \partial_{t}\left[K_{F}^{r}(t, \mathbf{q})\right] \dot{\mathbf{q}}=- \int_{\widetilde{\Gamma}_{2}(t)} \rho_{F}\left[D \widetilde{\varphi}^{r}\right]\left[D \widetilde{\varphi}^{r}\right]^{T} \dot{\mathbf{q}}\left(\widetilde{\mathbf{v}}^{d} \cdot \mathbf{n}\right) \mathrm{d} \Gamma_{x} \\
&+\int_{\widetilde{\Gamma}_{2}(t)} \rho_{F}\left(\widetilde{\varphi}^{r}(\dot{\mathbf{q}} \cdot \widetilde{\mathbf{A}})+\left(\widetilde{\varphi}^{r} \cdot \dot{\mathbf{q}}\right) \widetilde{\mathbf{A}}\right) \mathrm{d} \Gamma_{x}+2 \int_{\widetilde{\Gamma}_{2}(t)} \rho_{F}\left[\widetilde{V}^{r}\right]^{T} \mathbf{n}\left(\mathbf{n} \cdot\left[\widetilde{V}^{r}\right] \dot{\mathbf{q}}\right)\left(\widetilde{\mathbf{v}}^{d} \cdot \mathbf{n}\right) \mathrm{d} \Gamma_{x} \\
& \quad-2 \int_{\widetilde{\Gamma}_{2}(t)} \rho_{F} \mathcal{H}\left(\left[\widetilde{V}^{r}\right]^{T} \mathbf{n}\left(\widetilde{\varphi}^{r} \cdot \dot{\mathbf{q}}\right)+\widetilde{\varphi}^{r}\left(\dot{\mathbf{q}} \cdot\left[\widetilde{V}^{r}\right]^{T} \mathbf{n}\right)\right)\left(\widetilde{\mathbf{v}}^{d} \cdot \mathbf{n}\right) \mathrm{d} \Gamma_{x}
\end{aligned}
$$

Upon the change of variables $\mathbf{x}:=\mathcal{R}(\boldsymbol{\alpha}) \widetilde{\mathbf{x}}+\mathbf{h}$, the identity above turns into:

$$
\partial_{t}\left[K_{F}^{r}(t, \mathbf{q})\right] \dot{\mathbf{q}}=\left[M_{s y m}^{F / d}(t, \mathbf{q})\right] \dot{\mathbf{q}}
$$

the $6 \times 6$ symmetric matrix $\left[M_{\text {sym }}^{F / d}(t, \mathbf{q})\right]$ being given in (D.4). We compute as well that, on $\widetilde{\Gamma}_{2}(t): \partial_{t} \widetilde{B}^{d}\left(t, \mathbf{q}, \widetilde{\boldsymbol{\Lambda}}^{-1}(t, \mathbf{q})\right)=\dot{\overrightarrow{\mathbf{v}}}^{d} \cdot \mathbf{n}-\nabla_{\Gamma}\left(\widetilde{\mathbf{v}}^{d} \cdot \mathbf{n}\right) \cdot \widetilde{\mathbf{v}}^{d}-\kappa_{1}\left(\widetilde{\mathbf{v}}^{d} \cdot \boldsymbol{\tau}_{1}\right)^{2}-\kappa_{2}\left(\widetilde{\mathbf{v}}^{d}\right.$. $\left.\boldsymbol{\tau}_{2}\right)^{2}$. Since $\nabla_{\Gamma} \widetilde{b}^{d}(t, \mathbf{q}) \cdot \widetilde{\mathbf{v}}^{d}=\nabla_{\Gamma}\left(\widetilde{\mathbf{v}}^{d} \cdot \mathbf{n}\right) \cdot \widetilde{\mathbf{v}}^{d}$, we obtain $\partial_{t} \widetilde{B}^{d}\left(t, \mathbf{q}, \widetilde{\boldsymbol{\Lambda}}^{-1}(t, \mathbf{q})\right)-$ $\nabla_{\Gamma} \widetilde{b}^{d}(t, \mathbf{q}) \cdot \widetilde{\mathbf{v}}^{d}=\dot{\widetilde{\mathbf{v}}}^{d} \cdot \mathbf{n}-2 \nabla_{\Gamma}\left(\widetilde{\mathbf{v}}^{d} \cdot \mathbf{n}\right) \cdot \widetilde{\mathbf{v}}^{d}-\kappa_{1}\left(\widetilde{\mathbf{v}}^{d} \cdot \boldsymbol{\tau}_{1}\right)^{2}-\kappa_{2}\left(\widetilde{\mathbf{v}}^{d} \cdot \boldsymbol{\tau}_{2}\right)^{2}$. Applying 
formula (4.3), we get:

$$
\begin{aligned}
\partial_{t} \mathbf{K}_{F}^{r, d}(t, \mathbf{q})=-\int_{\widetilde{\Gamma}_{2}(t)} \rho_{F}\left(\widetilde{\mathbf{v}}^{d} \cdot \mathbf{n}\right)\left[D \widetilde{\varphi}^{r}\right]\left(\nabla \widetilde{\varphi}^{d}\right)^{T} \mathrm{~d} \Gamma_{x} \\
+2 \int_{\widetilde{\Gamma}_{2}(t)} \rho_{F}\left[\widetilde{V}^{r}\right]^{T} \mathbf{n}\left(\widetilde{\mathbf{v}}^{d} \cdot \mathbf{n}\right)^{2} \mathrm{~d} \Gamma_{x}-2 \int_{\widetilde{\Gamma}_{2}(t)} \rho_{F} \mathcal{H}\left(\widetilde{\varphi}^{d}\left[\widetilde{V}^{r}\right]^{T} \mathbf{n}+\left(\widetilde{\mathbf{v}}^{d} \cdot \mathbf{n}\right) \widetilde{\varphi}^{r}\right) \widetilde{\mathbf{v}}^{d} \cdot \mathbf{n} \mathrm{d} \Gamma_{x} \\
+\int_{\widetilde{\Gamma}_{2}(t)} \rho_{F}\left(\widetilde{\varphi}^{d} \widetilde{\mathbf{A}}+\left(\dot{\widetilde{\mathbf{v}}}^{d} \cdot \mathbf{n}-2 \nabla_{\Gamma}\left(\widetilde{\mathbf{v}}^{d} \cdot \mathbf{n}\right) \cdot \widetilde{\mathbf{v}}^{d}\right.\right. \\
\left.\left.\quad-\kappa_{1}\left(\widetilde{\mathbf{v}}^{d} \cdot \boldsymbol{\tau}_{1}\right)^{2}-\kappa_{2}\left(\widetilde{\mathbf{v}}^{d} \cdot \boldsymbol{\tau}_{2}\right)^{2}\right) \widetilde{\boldsymbol{\varphi}}^{r}\right) \mathrm{d} \Gamma_{x} .
\end{aligned}
$$

The change of variables $\mathbf{x}:=\mathcal{R}(\boldsymbol{\alpha}) \widetilde{\mathbf{x}}+\mathbf{h}$ leads to:

$$
\begin{gathered}
\partial_{t} \mathbf{K}_{F}^{r, d}(t, \mathbf{q})=-\int_{\Gamma_{2}(t, \mathbf{q})} \rho_{F}\left(\mathbf{v}^{d} \cdot \mathbf{n}\right)\left[D \varphi^{r}\right]\left(\nabla \varphi^{d}\right)^{T} \mathrm{~d} \Gamma_{x} \\
+2 \int_{\Gamma_{2}(t, \mathbf{q})} \rho_{F}\left[W^{r}\right]^{T} \mathbf{n}\left(\mathbf{v}^{d} \cdot \mathbf{n}\right)^{2} \mathrm{~d} \Gamma_{x}-2 \int_{\Gamma_{2}(t, \mathbf{q})} \rho_{F} \mathcal{H}\left(\varphi^{d}\left[W^{r}\right]^{T} \mathbf{n}+\left(\mathbf{v}^{d} \cdot \mathbf{n}\right) \boldsymbol{\varphi}^{r}\right) \mathbf{v}^{d} \cdot \mathbf{n} \mathrm{d} \Gamma_{x} \\
+\int_{\Gamma_{2}(t, \mathbf{q})} \rho_{F}\left(\varphi^{d} \mathbf{A}+\left(\dot{\mathbf{v}}^{d} \cdot \mathbf{n}-2 \nabla_{\Gamma}\left(\mathbf{v}^{d} \cdot \mathbf{n}\right) \cdot \mathbf{v}^{d}\right.\right. \\
\left.\left.\quad-\kappa_{1}\left(\mathbf{v}^{d} \cdot \boldsymbol{\tau}_{1}\right)^{2}-\kappa_{2}\left(\mathbf{v}^{d} \cdot \boldsymbol{\tau}_{2}\right)^{2}\right) \boldsymbol{\varphi}^{r}\right) \mathrm{d} \Gamma_{x}
\end{gathered}
$$

Notice that $-\kappa_{1}\left(\mathbf{v}^{d} \cdot \boldsymbol{\tau}_{1}\right)^{2}-\kappa_{2}\left(\mathbf{v}^{d} \cdot \boldsymbol{\tau}_{2}\right)^{2}$ can be replaced by $\Pi_{2}\left((t, \mathbf{q}), \mathbf{v}_{\Gamma}^{d}, \mathbf{v}_{\Gamma}^{d}\right)$ where $\Pi_{2}(t, \mathbf{q})$ is the second fondamental form on $\Gamma_{2}(t, \mathbf{q})$ and $\mathbf{v}_{\Gamma}^{d}:=\mathbf{v}^{d}-\left(\mathbf{v}^{d} \cdot \mathbf{n}\right) \mathbf{n}$ is the tangential part of $\mathbf{v}^{d}$. Following the same ideas we obtain:

$$
\partial_{\mathbf{q}} K_{F}^{d}(t, \mathbf{q})=-\int_{\Gamma_{1}} \rho_{F}\left|\nabla \varphi^{d}\right|^{2}\left[W^{r}\right]^{T} \mathbf{n} \mathrm{d} \Gamma_{x}
$$

Eventually we get upon a change of variables:

$$
\mathcal{P}(t, \mathbf{q})=-\int_{S_{0}}\left(\rho_{S}^{0}-\rho_{F}|\operatorname{det}[D \boldsymbol{\Lambda}(t, \mathbf{q}, \mathbf{x})]|\right) G(\boldsymbol{\Lambda}(t, \mathbf{q}, \mathbf{x})) \mathrm{d} \mathbf{x} .
$$

Applying formula (5.16) page 175 in Ref. [18], that is $\partial_{q_{i}} \operatorname{det}[D \boldsymbol{\Lambda}(t, \mathbf{q})]=\operatorname{det}[D \boldsymbol{\Lambda}(t, \mathbf{q})] \operatorname{div}\left[\partial_{q_{i}} \boldsymbol{\Lambda}(t, \mathbf{q})\right]$ we obtain:

$$
\begin{aligned}
\partial_{q_{i}} \mathcal{P}(t, \mathbf{q})=\int_{S(t, \mathbf{q})} \rho_{F} \operatorname{div}\left(\partial_{q_{i}} \mathbf{\Lambda}\left(t, \mathbf{q}, \boldsymbol{\Lambda}^{-1}(t, \mathbf{q}, \mathbf{x})\right) G(\mathbf{x}) \mathrm{d} \mathbf{x}\right. \\
\quad-\int_{S(t, \mathbf{q})}\left(\rho_{S}(\mathbf{x})-\rho_{F}\right) \nabla G(\mathbf{x}) \cdot \partial_{q_{i}} \boldsymbol{\Lambda}\left(t, \mathbf{q}, \boldsymbol{\Lambda}^{-1}(t, \mathbf{q}, \mathbf{x})\right) \mathrm{d} \Gamma_{x} .
\end{aligned}
$$

But $\partial_{q_{i}} \boldsymbol{\Lambda}\left(t, \mathbf{q}, \boldsymbol{\Lambda}^{-1}(t, \mathbf{q}, \mathbf{x})\right)=\mathbf{v}_{i}^{r}$ and $\operatorname{div}\left(\mathbf{v}_{i}^{r}\right)=0$ for all $i=1, \ldots, 6$. Hence it comes:

$$
\partial_{q_{i}} \mathcal{P}(t, \mathbf{q})=\int_{S(t, \mathbf{q})}\left(\rho_{S}(\mathbf{x})-\rho_{F}\right) g\left[W^{r}\right]^{T} \mathbf{e}_{3} \mathrm{~d} \mathbf{x},
$$

and this expression matchs that of $\mathbf{F}^{b}(t, \mathbf{q})$ given by (D.5). All the terms arising in the ODE (6.1) have now been computed and the proof of Theorem 2 is complete. 


\section{Future DiRECTIONS}

A natural continuation of the results presented in this paper would be to perform $3 \mathrm{~d}$ numerical simulations using the formula given in Theorem 2. This work is in progress.

About control, an interesting question is: for a given rigid motion of the swimmer in the fluid, is there a deformation that yields this motion? This problem is known as tracking problem. Equation (5.4) is then considered as an ODE in $\phi$, in which $\mathbf{q}, \dot{\mathbf{q}}$ and $\ddot{\mathbf{q}}$ are given parameters. The uniqueness in the Cauchy-Lipschitz Theorem ensures that if such a deformation exists, when replugging it into the same ODE as parameter, the solution cannot be anything but the initial targeting motion. The theoretical problem of controlling an ellipsoid (modelling a submarine) in a potential flow was recently tackled by T. Chambrion and M. Sigalotti[9] using tools of geometric control.

At last, a sharp asymptotic study of the motion when two bodies get close and finally collide remains to be done.

\section{Appendix A. Euler Angles}

Let be $\mathcal{A}:=]-\pi, \pi[\times]-\pi / 2, \pi / 2[\times]-\pi, \pi[$ and $\widetilde{\mathcal{A}}:=]-\pi, \pi[\times]-\pi / 4, \pi / 4[\times]-\pi, \pi[$ and define the function:

$$
\begin{aligned}
\mathcal{R}: \mathcal{A} & \rightarrow S O(3) \\
\boldsymbol{\alpha}=\left(\alpha_{1}, \alpha_{2}, \alpha_{3}\right)^{T} & \mapsto \mathcal{R}(\boldsymbol{\alpha}):=\left[\begin{array}{ccc}
c_{2} c_{3} & -c_{2} s_{3} & -s_{2} \\
-s_{1} s_{2} c_{3}+c_{1} s_{3} & s_{1} s_{2} s_{3}+c_{1} c_{3} & -s_{1} c_{2} \\
c_{1} s_{2} c_{3}+s_{1} s_{3} & -c_{1} s_{2} s_{3}+s_{1} c_{3} & c_{1} c_{2}
\end{array}\right],
\end{aligned}
$$

where $c_{i}$ stands for $\cos \left(\alpha_{i}\right)$ and $s_{i}$ for $\sin \left(\alpha_{i}\right)$. The pair $(\mathcal{A}, \mathcal{R})$ is an analytic chart from $\mathcal{A}$ onto a neighborhood of $\left[\mathbb{I}_{3}\right]$ in $S O(3)$ such that $\mathcal{R}\left(\mathbf{0}_{3}\right)=\left[\mathbb{I}_{3}\right]$. The coordinates $\alpha_{i}$ are the so-called Euler angles with the "xyz" (pitch-roll-yaw) convention. Introducing the vectors $\boldsymbol{\omega}_{1}(\boldsymbol{\alpha}):=(1,0,0)^{T}, \boldsymbol{\omega}_{2}(\boldsymbol{\alpha}):=\left(0, \cos \left(\alpha_{1}\right), \sin \left(\alpha_{1}\right)\right)^{T}$ and $\boldsymbol{\omega}_{3}(\boldsymbol{\alpha}):=\left(-\sin \left(\alpha_{2}\right),-\sin \left(\alpha_{1}\right) \cos \left(\alpha_{2}\right), \cos \left(\alpha_{1}\right) \cos \left(\alpha_{2}\right)\right)^{T}$, we have the relations, easy to prove:

$$
\frac{\partial \mathcal{R}}{\partial \alpha_{i}}(\boldsymbol{\alpha}) \mathcal{R}(\boldsymbol{\alpha})^{T} \mathbf{x}=\boldsymbol{\omega}_{i}(\boldsymbol{\alpha}) \wedge \mathbf{x}, \quad \forall \mathbf{x} \in \mathbb{R}^{3}, \forall \boldsymbol{\alpha} \in \mathcal{A}, \forall i=1,2,3
$$

We denote:

$$
[\omega(\boldsymbol{\alpha})]:=\left[\begin{array}{ccc}
1 & 0 & -\sin \left(\alpha_{2}\right) \\
0 & \cos \left(\alpha_{1}\right) & -\sin \left(\alpha_{2}\right) \cos \left(\alpha_{2}\right) \\
0 & \sin \left(\alpha_{1}\right) & \cos \left(\alpha_{1}\right) \cos \left(\alpha_{2}\right)
\end{array}\right]
$$

We can compute the derivatives of the vectors $\boldsymbol{\omega}_{k}(\boldsymbol{\alpha})$ :

$$
\frac{\partial \boldsymbol{\omega}_{k}}{\partial \alpha_{j}}(\boldsymbol{\alpha})= \begin{cases}\boldsymbol{\omega}_{j}(\boldsymbol{\alpha}) \wedge \boldsymbol{\omega}_{k}(\boldsymbol{\alpha}), & \text { if } 1 \leq j<k \leq 3 \\ \mathbf{0}_{3}, & \text { if } 1 \leq k \leq j \leq 3\end{cases}
$$

The differential of the matrix $[\omega(\boldsymbol{\alpha})]$ is a third rank tensor given by:

(A.1c) $\partial_{\boldsymbol{\alpha}}[\omega(\boldsymbol{\alpha})]=\mathbf{e}_{2} \otimes\left(\boldsymbol{\omega}_{1} \wedge \boldsymbol{\omega}_{2}\right) \otimes \mathbf{e}_{1}+\mathbf{e}_{3} \otimes\left(\boldsymbol{\omega}_{1} \wedge \boldsymbol{\omega}_{3}\right) \otimes \mathbf{e}_{1}+\mathbf{e}_{3} \otimes\left(\boldsymbol{\omega}_{2} \wedge \boldsymbol{\omega}_{3}\right) \otimes \mathbf{e}_{2}$.

The $6 \times 6$ block-diagonal matrix $[\widetilde{\omega}(\boldsymbol{\alpha})]$ is defined by :

$$
[\widetilde{\omega}(\boldsymbol{\alpha})]:=\operatorname{diag}\left([\omega(\boldsymbol{\alpha})],\left[\mathbb{I}_{3}\right]\right),
$$

and we have $\operatorname{det}[\widetilde{\omega}(\boldsymbol{\alpha})]=\cos \left(\alpha_{2}\right)$. 


\section{Appendix B. Deformation spaces}

Let $\mathbb{B}$ be a ball in $\mathbb{R}^{3}$, centered at the origin an large enough to contain all the initial regions $S_{0}^{i}-\mathbf{h}_{0}^{i}$ for $i=1, \ldots, n$. Let $C_{0}^{m}\left(\mathbb{B}, \mathbb{R}^{3}\right)(m \geq 0)$ be the space of the functions $\mathbf{F}=\left(F_{1}, F_{2}, F_{3}\right)^{T}$ from $\mathbb{R}^{3}$ into $\mathbb{R}^{3}, m$-times continuously differentiable and that vanish outside $\bar{B}$. This space, endowed with the norm:

$$
\|\mathbf{F}\|_{m, \infty}: \sup _{\substack{\gamma_{1}+\gamma_{2}+\gamma_{3} \leq m \\ \gamma_{1}, \gamma_{2}, \gamma_{3} \in \mathbb{N}}}\left(\sup _{i=1,2,3}\left\|\frac{\partial^{\gamma_{1}+\gamma_{2}+\gamma_{3}} F_{i}}{\partial \gamma_{1} x_{1} \partial^{\gamma_{2}} x_{2} \partial^{\gamma_{3}} x_{3}}\right\|_{L^{\infty}(\mathbb{B})}\right),
$$

is a Banach space. If $m \geq 1$ and $\|\mathbf{F}\|_{1, \infty}<1$, a fixed point argument ensures that $\left[\mathbb{I}_{3}\right]+\mathbf{F}$ is a $C^{m}$-diffeomorphism from $\mathbb{B}$ onto $\mathbb{B}$. The functions of $C_{0}^{m}\left(\mathbb{B}, \mathbb{R}^{3}\right)$ obviously belong to $C^{m, \infty}\left(\mathbb{R}^{3}, \mathbb{R}^{3}\right)$, the space of the $m$-times continuously differentiable functions, uniformly bounded in $\mathbb{R}^{3}$ as well as all of their derivatives. This space is also a Banach space endowed with the norm $\|\cdot\|_{m, \infty}$. For any function $\mathbf{F}:=\left(F_{1}, F_{2}, F_{3}\right)^{T} \in C^{m, \infty}\left(\mathbb{R}^{3}, \mathbb{R}^{3}\right)$, the Jacobian matrix $[D \mathbf{F}]$ can be considered as a function of $C^{m-1, \infty}\left(\mathbb{R}^{3}, \mathbb{R}^{3}\right)^{3}$ since each row $\nabla F_{i}$ of the matrix is in $C^{m-1, \infty}\left(\mathbb{R}^{3}, \mathbb{R}^{3}\right)$. More generally, if $[F(\mathbf{x})]$ is a matrix for which the rows are three functions $\mathbf{F}_{1}, \mathbf{F}_{2}$ and $\mathbf{F}_{3}$ lying in $C^{m, \infty}\left(\mathbb{R}^{3}, \mathbb{R}^{3}\right)$, we will consider that $[F] \in C^{m, \infty}\left(\mathbb{R}^{3}, \mathbb{R}^{3}\right)^{3}$ and denote

$$
\|[F]\|_{m, \infty, 3}:=\sup _{i=1,2,3}\left\|\mathbf{F}_{i}\right\|_{m, \infty}
$$

Obviously, the space $C^{m, \infty}\left(\mathbb{R}^{3}, \mathbb{R}^{3}\right)^{3}$ endowed with the norm $\|\cdot\|_{m, \infty, 3}$ is a Banach space.

Denoting $\boldsymbol{\phi}:=\left(\boldsymbol{\phi}^{1}, \ldots, \boldsymbol{\phi}^{n}\right)^{T} \in C_{0}^{m}\left(\mathbb{B}, \mathbb{R}^{3}\right)^{n}$ and $\boldsymbol{\psi}:=\left(\boldsymbol{\psi}^{1}, \ldots, \boldsymbol{\psi}^{n}\right)^{T} \in C_{0}^{m}\left(\mathbb{B}, \mathbb{R}^{3}\right)^{n}$ with $\phi^{i}:=\left(\phi_{1}^{i}, \phi_{2}^{i}, \phi_{3}^{i}\right)^{T} \in C_{0}^{m}\left(\mathbb{B}, \mathbb{R}^{3}\right)$ and $\psi^{i}:=\left(\psi_{1}^{i}, \psi_{2}^{i}, \psi_{3}^{i}\right) \in C_{0}^{m}\left(\mathbb{B}, \mathbb{R}^{3}\right)$, we define the deformation fiber bundle:

$$
\begin{gathered}
\mathcal{D}^{n, m}:=\left\{(\boldsymbol{\phi}, \boldsymbol{\psi}) \in C_{0}^{m}\left(\mathbb{B}, \mathbb{R}^{3}\right)^{n} \times C_{0}^{m}\left(\mathbb{B}, \mathbb{R}^{3}\right)^{n}:\left\|\phi^{i}\right\|_{1, \infty}<1,\right. \\
\int_{S_{0}^{i}} \rho_{S}^{0}(\mathbf{x}) \boldsymbol{\psi}^{i}\left(\mathbf{x}-\mathbf{h}_{0}^{i}\right) \mathrm{d} \mathbf{x}=\mathbf{0}_{3}, \int_{S_{0}^{i}} \rho_{S}^{0}(\mathbf{x})\left(\mathbf{x}+\phi^{i}\left(\mathbf{x}-\mathbf{h}_{0}^{i}\right)\right) \wedge \boldsymbol{\psi}^{i}\left(\mathbf{x}-\mathbf{h}_{0}^{i}\right)=\mathbf{0}_{3} \\
\quad \text { and if } \Omega \text { is bounded: } \\
\left.\sum_{i=1}^{n} \int_{S_{0}^{i}} \operatorname{div}\left(\boldsymbol{\psi}^{i}\left(\mathbf{x}-\mathbf{h}_{0}^{i}\right)\right)\left|\operatorname{det}\left(\left[\mathbb{I}_{3}\right]+\left[D \phi^{i}\left(\mathbf{x}-\mathbf{h}_{0}^{i}\right)\right]\right)\right|=0, \forall i=1, \ldots, n\right\} .
\end{gathered}
$$

Remark that $\mathcal{D}^{n, m}$ does not contain any function that reduces to a rigid displacement on one $S_{0}^{i}$.

\section{Appendix C. Global and local coordinates}

Global coordinates. According to relation (2.1), the location and the shape of the $i$-th body are given by a pair $\mathbf{Q}^{i}:=\left(\left[R^{i}\right], \mathbf{h}^{i}\right) \in S O(3) \times \mathbb{R}^{3}$ and a function $\phi^{i}:=\left(\phi_{1}^{i}, \phi_{2}^{i}, \phi_{3}^{i}\right)^{T} \in C_{0}^{m}\left(\mathbb{B}, \mathbb{R}^{3}\right)$. We denote $\mathbf{Q}:=\left(\mathbf{Q}^{1}, \ldots, \mathbf{Q}^{n}\right):=$ $\left(\left(\left[R^{1}\right], \mathbf{h}^{1}\right), \ldots,\left(\left[R^{n}\right], \mathbf{h}^{n}\right)\right) \in\left(S O(3) \times \mathbb{R}^{3}\right)^{n}$ and $\boldsymbol{\phi}:=\left(\phi^{1}, \ldots, \phi^{n}\right)^{T}:=\left(\phi_{1}^{1}, \phi_{2}^{1}, \phi_{3}^{1}, \ldots, \phi_{1}^{n}, \phi_{2}^{n}, \phi_{3}^{n}\right)^{T} \in$ $C_{0}^{m}\left(\mathbb{B}, \mathbb{R}^{3}\right)^{n}$. The $S O(3)$ group being an infinitely differentiable 3 -dimensional submanifold of $\mathcal{M}(3)$ (the vector space of the $3 \times 3$ matrices), $\left(S O(3) \times \mathbb{R}^{3}\right)^{n}$ is a $6 n$-dimensional submanifold of $\left(\mathcal{M}(3) \times \mathbb{R}^{3}\right)^{n}$. We define $\mathcal{Q}^{n, m}$ as being the open subset of $(\mathbf{Q}, \phi) \in\left(S O(3) \times \mathbb{R}^{3}\right)^{n} \times C_{0}^{m}\left(\mathbb{B}, \mathbb{R}^{3}\right)^{n}$ for which any body do not touch or overlap another body or the boundary of the fluid region. The connected component 
set of $\mathcal{Q}^{n, m}$ containing the pair $\left(\mathbf{Q}_{0}, \mathbf{\Phi}_{0}\right)$ in which $\mathbf{Q}_{0}:=\left(\left(\left[\mathbb{I}_{3}\right], \mathbf{h}_{0}^{1}\right), \ldots,\left(\left[\mathbb{I}_{3}\right], \mathbf{h}_{0}^{n}\right)\right) \in$ $\left(S O(3) \times \mathbb{R}^{3}\right)^{n}$ and $\phi_{0}:=\left(\left[\mathbb{O}_{3}\right], \ldots,\left[\mathbb{O}_{3}\right]\right) \in C_{0}^{m}\left(\mathbb{B}, \mathbb{R}^{3}\right)^{n}$ is the set of all the reachable (or allowable) configurations. It is denoted $\mathcal{Q}_{a l}^{n, m}$. We denote $T \mathcal{Q}^{n, m}$ the tangent bundle of $\mathcal{Q}^{n, m}$ and we introduce the subset of $T \mathcal{Q}_{a l}^{n, m}$ of all the allowable positions, velocities, deformations and deformations rate of the bodies:

$$
T \mathcal{Q}_{a l}^{n, m}:=\left\{((\mathbf{Q}, \boldsymbol{\phi}),(\dot{\mathbf{Q}}, \dot{\phi})) \in T \mathcal{Q}^{n, m}:(\mathbf{Q}, \phi) \in \mathcal{Q}_{a l}^{n, m},(\phi, \dot{\boldsymbol{\phi}}) \in \mathcal{D}^{n, m}\right\} .
$$

Remark that this set keep a structure of fiber bundle. The elements $(\mathbf{Q}, \boldsymbol{\phi}) \in \mathcal{Q}_{a l}^{n, m}$ are called the global coordinates.

Local coordinates. Let us denote $\boldsymbol{\alpha}^{i}:=\left(\alpha_{1}^{i}, \alpha_{2}^{i}, \alpha_{3}^{i}\right) \in \mathcal{A}$ the Euler angles of the $i$-th body (the Euler angles are detailed in A) and introduce the function:

$$
\begin{aligned}
\mathfrak{F}: & \left(\mathcal{A} \times \mathbb{R}^{3}\right)^{n} \times C_{0}^{m}\left(\mathbb{B}, \mathbb{R}^{3}\right)^{n} \rightarrow\left(S O(3) \times \mathbb{R}^{3}\right)^{n} \times C_{0}^{m}\left(\mathbb{B}, \mathbb{R}^{3}\right)^{n} \\
\quad\left(\left(\boldsymbol{\alpha}^{1}, \mathbf{h}^{1}\right), \ldots,\left(\boldsymbol{\alpha}^{n}, \mathbf{h}^{n}\right), \boldsymbol{\phi}\right) & \mapsto\left(\left(\mathcal{R}\left(\boldsymbol{\alpha}^{1}\right), \mathbf{h}^{1}\right), \ldots,\left(\mathcal{R}\left(\boldsymbol{\alpha}^{n}\right), \mathbf{h}^{n}\right), \boldsymbol{\phi}\right) .
\end{aligned}
$$

We set $\mathfrak{F}_{1}\left(\left(\boldsymbol{\alpha}^{1}, \mathbf{h}^{1}\right), \ldots,\left(\boldsymbol{\alpha}^{n}, \mathbf{h}^{n}\right), \boldsymbol{\phi}\right):=\left(\mathcal{R}\left(\boldsymbol{\alpha}^{1}\right), \mathbf{h}^{1}\right), \ldots,\left(\mathcal{R}\left(\boldsymbol{\alpha}^{n}\right), \mathbf{h}^{n}\right), \mathfrak{F}_{2}\left(\left(\boldsymbol{\alpha}^{1}, \mathbf{h}^{1}\right), \ldots,\left(\boldsymbol{\alpha}^{n}, \mathbf{h}^{n}\right), \boldsymbol{\phi}\right):=$ $\phi$ and $\mathfrak{F}:=\left(\mathfrak{F}_{1}, \mathfrak{F}_{2}\right)$. The elements

$$
(\mathbf{q}, \boldsymbol{\phi}):=\left(\alpha_{1}^{1}, \alpha_{2}^{1}, \alpha_{3}^{1}, h_{1}^{1}, h_{2}^{1}, h_{3}^{1}, \ldots, \alpha_{1}^{n}, \alpha_{2}^{n}, \alpha_{3}^{n}, h_{1}^{n}, h_{2}^{n}, h_{3}^{n}, \boldsymbol{\phi}\right),
$$

of the open subset $\mathfrak{Q}_{a l}^{n, m}:=\left(\mathcal{A} \times \mathbb{R}^{3}\right)^{n} \times C_{0}^{m}\left(\mathbb{B}, \mathbb{R}^{3}\right)^{n} \cap \mathfrak{F}^{-1}\left(\mathcal{Q}_{a l}^{n, m}\right)$ of $\left(\mathbb{R}^{3} \times \mathbb{R}^{3}\right)^{n} \times$ $C_{0}^{m}\left(\mathbb{B}, \mathbb{R}^{3}\right)^{n}$ will also be generically denoted $(\mathbf{q}, \boldsymbol{\phi}):=\left(q_{1}^{1}, q_{2}^{1}, q_{3}^{1}, \ldots, q_{1}^{n}, q_{2}^{n}, q_{3}^{n}, \boldsymbol{\phi}\right)$. We set also $\widetilde{\mathfrak{Q}}_{a l}^{n, m}:=\left(\widetilde{\mathcal{A}} \times \mathbb{R}^{3}\right)^{n} \times C_{0}^{m}\left(\mathbb{B}, \mathbb{R}^{3}\right)^{n} \cap \mathfrak{F}^{-1}\left(\mathcal{Q}_{a l}^{n, m}\right) \subset \mathfrak{Q}_{a l}^{n, m}$. The pairs $\left(\mathfrak{Q}_{a l}^{n, m}, \mathfrak{F}\right)$ or $\left(\widetilde{\mathfrak{Q}}_{a l}^{n, m}, \mathfrak{F}\right)$ are local analytic charts of $\mathcal{Q}_{a l}^{n, m}$ in a neighborhood of $\left(\mathbf{Q}_{0}, \phi_{0}\right)$ such that $\mathfrak{F}\left(\mathbf{q}_{0}, \boldsymbol{\phi}_{0}\right)=\left(\mathbf{Q}_{0}, \boldsymbol{\phi}_{0}\right)$ where $\mathbf{q}_{0}:=\left(\left(\mathbf{0}_{3}, \mathbf{h}_{0}^{1}\right), \ldots,\left(\mathbf{0}_{3}, \mathbf{h}_{0}^{n}\right)\right)^{T} \in$ $\mathbb{R}^{6 n}$. We denote

$$
\begin{aligned}
& T \mathfrak{Q}_{a l}^{n, m}:=\left\{((\mathbf{q}, \boldsymbol{\phi}),(\dot{\mathbf{q}}, \dot{\boldsymbol{\phi}})) \in \mathfrak{Q}_{a l}^{n, m} \times\left(\mathbb{R}^{6^{n}} \times C_{0}^{m}\left(\mathbb{B}, \mathbb{R}^{3}\right)^{n}\right):(\boldsymbol{\phi}, \dot{\boldsymbol{\phi}}) \in \mathcal{D}^{n, m}\right\}, \\
& T \widetilde{\mathfrak{Q}}_{a l}^{n, m}:=\left\{((\mathbf{q}, \boldsymbol{\phi}),(\dot{\mathbf{q}}, \dot{\boldsymbol{\phi}})) \in \widetilde{\mathfrak{Q}}_{a l}^{n, m} \times\left(\mathbb{R}^{6^{n}} \times C_{0}^{m}\left(\mathbb{B}, \mathbb{R}^{3}\right)^{n}\right):(\boldsymbol{\phi}, \dot{\boldsymbol{\phi}}) \in \mathcal{D}^{n, m}\right\} .
\end{aligned}
$$

The elements $(\mathbf{q}, \phi) \in \mathfrak{Q}_{a l}^{m}$ are called the local coordinates.

\section{Appendix D. Definitions of some tensors, matrices And VeCtors}

Under the assumptions of Theorem 2, we denote $\boldsymbol{\Phi}(t):=\left[\mathbb{I}_{3}\right]+\phi(t)$, for all $0 \leq t<T$. For all $(t, \mathbf{q}) \in[0, T) \times \mathbb{R}^{6}$ such that $(\mathbf{q}, \boldsymbol{\phi}(t)) \in \mathfrak{Q}_{a l}^{1,2}, \Omega(t, \mathbf{q})$ and $S(t, \mathbf{q})$ stand for the region of the fluid and the region of the swimmer respectively and $\Gamma_{2}(t, \mathbf{q}):=\partial S(t, \mathbf{q})$. We define the following vector fields for all $\mathbf{x} \in S(t, \mathbf{q})$ :

$$
\begin{aligned}
& \mathbf{v}^{d}(t, \mathbf{q}, \mathbf{x}):=\mathcal{R}(\boldsymbol{\alpha}) \dot{\boldsymbol{\phi}}\left(t, \boldsymbol{\Phi}^{-1}\left(t, \mathcal{R}(\boldsymbol{\alpha})^{T}(\mathbf{x}-\mathbf{h})\right)\right), \\
& \dot{\mathbf{v}}^{d}(t, \mathbf{q}, \mathbf{x}):=\mathcal{R}(\boldsymbol{\alpha}) \ddot{\boldsymbol{\phi}}\left(t, \Phi^{-1}\left(t, \mathcal{R}(\boldsymbol{\alpha})^{T}(\mathbf{x}-\mathbf{h})\right)\right),
\end{aligned}
$$

where $\ddot{\boldsymbol{\phi}}$ stands for $\partial_{t t} \boldsymbol{\phi}$. Also for all $\mathbf{x} \in S(t, \mathbf{q})$, the vector $\mathbf{A}(t, \mathbf{q}, \mathbf{x})$ is given by:

$$
A_{i}(t, \mathbf{q}, \mathbf{x}):= \begin{cases}-\boldsymbol{\omega}_{i} \wedge(\mathbf{x}-\mathbf{h}) \cdot \nabla_{\Gamma}\left(\mathbf{v}^{d}(t, \mathbf{q}, \mathbf{x}) \cdot \mathbf{n}\right) & \text { if } i=1,2,3 \\ -\mathbf{e}_{i-3} \cdot \nabla_{\Gamma}\left(\mathbf{v}^{d}(t, \mathbf{q}, \mathbf{x}) \cdot \mathbf{n}\right) & \text { if } i=4,5,6\end{cases}
$$


We define the $3 \times 6$ matrix $\left[W^{r}(t, \mathbf{q}, \mathbf{x})\right]$ on $\Gamma_{1} \cup S(t, \mathbf{q})$ by:

(D.1a) $\forall \mathbf{x} \in \Gamma_{1}, \quad W_{i j}^{r}(t, \mathbf{q}, \mathbf{x}):= \begin{cases}-\boldsymbol{\omega}_{j} \wedge(\mathbf{x}-\mathbf{h}) \cdot \mathbf{e}_{i} & \text { if } 1 \leq i, j \leq 3, \\ -1 & \text { if } i=j-3, j=4,5,6, \\ 0 & \text { otherwise, }\end{cases}$

$$
\forall \mathbf{x} \in S(t, \mathbf{q}), \quad W_{i j}^{r}(t, \mathbf{q}, \mathbf{x}):= \begin{cases}\boldsymbol{\omega}_{j} \wedge(\mathbf{x}-\mathbf{h}) \cdot \mathbf{e}_{i} & \text { if } 1 \leq i, j \leq 3, \\ 1 & \text { if } i=j-3, j=4,5,6, \\ 0 & \text { otherwise. }\end{cases}
$$

We set for all $i=1, \ldots, 6$ and all $\mathbf{x} \in S(t, \mathbf{q}): \mathbf{v}_{i}^{r}(t, \mathbf{q}, \mathbf{x}):=\left[W^{r}(t, \mathbf{q}, \mathbf{x})\right] \mathbf{f}_{i}$ where $\left\{\mathbf{f}_{1}, \ldots, \mathbf{f}_{6}\right\}$ is the canonical basis of $\mathbb{R}^{6}$. The potential functions $\varphi_{i}^{r}(t, \mathbf{q})(i=$ $1, \ldots, n)$ and $\varphi^{d}(t, \mathbf{q})$ are harmonic in $\Omega(t, \mathbf{q})$ and satisfy the Neumann boundary conditions:

$$
\begin{aligned}
& \partial_{\mathbf{n}} \varphi_{i}^{r}(t, \mathbf{q})=0 \text { on } \Gamma_{1} \quad \text { and } \quad \partial_{\mathbf{n}} \varphi_{i}^{r}(t, \mathbf{q})=\mathbf{v}_{i}^{r}(t, \mathbf{q}) \cdot \mathbf{n} \quad \text { on } \Gamma_{2}(t, \mathbf{q}) \text {, } \\
& \partial_{\mathbf{n}} \varphi^{d}(t, \mathbf{q})=0 \text { on } \Gamma_{1} \quad \text { and } \quad \partial_{\mathbf{n}} \varphi^{d}(t, \mathbf{q})=\mathbf{v}^{d}(t, \mathbf{q}) \cdot \mathbf{n} \quad \text { on } \Gamma_{2}(t, \mathbf{q}) \text {. }
\end{aligned}
$$

We set the function $\varphi^{r}(t, \mathbf{q}): \Omega(t, \mathbf{q}) \rightarrow \mathbb{R}^{6}$ by $\varphi^{r}(t, \mathbf{q}):=\left(\varphi_{1}^{r}(t, \mathbf{q}), \ldots, \varphi_{6}^{r}(t, \mathbf{q})\right)^{T}$. We introduce the following $3 \times 3$ symmetric matrices, where $\rho_{S}$ stand for the density of the bodies and $m$ its mass:

$$
\begin{aligned}
{[I(t, \mathbf{q})]:=} & \int_{S(t, \mathbf{q})} \rho_{S}(t, \mathbf{x})\left(|\mathbf{x}-\mathbf{h}|^{2}\left[\mathbb{I}_{3}\right]-(\mathbf{x}-\mathbf{h}) \otimes(\mathbf{x}-\mathbf{h})\right) \mathrm{d} \mathbf{x} \\
{\left[\partial_{t} I(t, \mathbf{q})\right]:=} & \int_{S(t, \mathbf{q})} \rho_{S}(t, \mathbf{x})\left(2(\mathbf{x}-\mathbf{h}) \cdot \mathbf{v}^{d}(t, \mathbf{q}, \mathbf{x})\left[\mathbb{I}_{3}\right]\right. \\
& \left.\quad-\mathbf{v}^{d}(t, \mathbf{q}, \mathbf{x}) \otimes(\mathbf{x}-\mathbf{h})-(\mathbf{x}-\mathbf{h}) \otimes \mathbf{v}^{d}(t, \mathbf{q}, \mathbf{x})\right) \mathrm{d} \mathbf{x},
\end{aligned}
$$

the $6 \times 6$ symmetric matrices (the matrix $[\widetilde{\omega}]$ arising below is defined by (A.2)):

$$
\begin{aligned}
{\left[K_{S}(t, \mathbf{q})\right] } & :=[\widetilde{\omega}(t, \mathbf{q})]^{T} \operatorname{diag}\left([I(t, \mathbf{q})], m\left[\mathbb{I}_{3}\right]\right)[\widetilde{\omega}(t, \mathbf{q})], \\
{\left[K_{F}(t, \mathbf{q})\right] } & :=\frac{1}{2} \int_{\Gamma_{2}(t, \mathbf{q})} \rho_{F}\left(\varphi^{r} \otimes\left[W^{r}\right]^{T} \mathbf{n}+\left[W^{r}\right]^{T} \mathbf{n} \otimes \boldsymbol{\varphi}^{r}\right) \mathrm{d} \Gamma_{x}, \\
{\left[M_{\text {sym }}^{S / d}(t, \mathbf{q})\right] } & :=[\widetilde{\omega}(\mathbf{q})]^{T} \operatorname{diag}\left(\left[\partial_{t} I(t, \mathbf{q})\right],\left[\mathbb{O}_{3}\right]\right)[\widetilde{\omega}(\mathbf{q})],
\end{aligned}
$$

and the following $6 \times 6$ matrices for all $\mathbf{x} \in \Gamma_{2}(t, \mathbf{q})$ :

$$
\begin{aligned}
& \widetilde{N}_{i j}(t, \mathbf{q}, \mathbf{x}):= \begin{cases}\left(x_{i}-h_{i}\right) n_{j}-n_{i}\left(x_{j}-h_{j}\right) & \text { if } 1 \leq i \leq j \leq 3, \\
\left(\mathbf{n} \wedge \mathbf{e}_{i-3}\right) \cdot \mathbf{e}_{j} & \text { if } i=4,5,6, j=1,2,3 \\
0 & \text { otherwise }\end{cases} \\
& {[N(t, \mathbf{q}, \mathbf{x})]:=[\widetilde{\omega}(\mathbf{q})]^{T}[\widetilde{N}(t, \mathbf{q}, \mathbf{x})][\widetilde{\omega}(\mathbf{q})] .}
\end{aligned}
$$

The entries of the $6 \times 6 \times 6$ third-rank tensors $\llbracket T_{s y m}^{S / r}(t, \mathbf{q}) \rrbracket$ and $\llbracket T_{s k e w}^{S / r}(t, \mathbf{q}) \rrbracket$ are:

$$
\begin{aligned}
&\left(T_{s y m}^{S / r}\right)_{j_{1} j_{2} j_{3}}(t, \mathbf{q}):= \\
& \begin{cases}\frac{1}{2}\left[\left(\boldsymbol{\omega}_{\min \left\{j_{2}, j_{3}\right\}} \wedge \boldsymbol{\omega}_{\max \left\{j_{2}, j_{3}\right\}}\right)[I] \boldsymbol{\omega}_{j_{1}}\right] & \text { if } 1 \leq j_{1}, j_{2}, j_{3} \leq 3, \\
0 & \text { otherwise, }\end{cases}
\end{aligned}
$$


and

$$
\begin{aligned}
& \left(T_{\text {skew }}^{S / r}\right)_{j_{1} j_{2} j_{3}}(t, \mathbf{q}):= \\
& \quad \begin{cases}\frac{1}{2}\left[\left(\boldsymbol{\omega}_{j_{2}} \wedge \boldsymbol{\omega}_{j_{1}}\right)[I] \boldsymbol{\omega}_{j_{3}}+\left(\boldsymbol{\omega}_{j_{3}} \wedge \boldsymbol{\omega}_{j_{1}}\right)[I] \boldsymbol{\omega}_{j_{2}}\right], & \text { if } 1 \leq j_{1}, j_{2}, j_{3} \leq 3 \\
0 & \text { otherwise }\end{cases}
\end{aligned}
$$

Let $\rho_{F}$ be the constant density of the fluid. The $6 \times 6 \times 6$ third-rank tensors $\llbracket T_{\text {sym }}^{F / r}(t, \mathbf{q}) \rrbracket$ and $\llbracket T_{\text {skew }}^{F / r}(t, \mathbf{q}) \rrbracket$ read respectively:

$$
\begin{aligned}
\llbracket T_{s y m}^{F / r}(t, \mathbf{q}) \rrbracket:=-\frac{1}{2} \int_{\Gamma_{1}} \rho_{F}\left[D \varphi^{r}\right]\left[D \varphi^{r}\right]^{T} \otimes\left(\left[W^{r}\right]^{T} \mathbf{n}\right) \mathrm{d} \Gamma_{x} \\
+\frac{1}{2} \int_{\Gamma_{2}(t, \mathbf{q})} \rho_{F} \boldsymbol{\varphi}^{r} \otimes\left([N(t, \mathbf{q}, \mathbf{x})]+[N(t, \mathbf{q}, \mathbf{x})]^{T}\right) \mathrm{d} \Gamma_{x}
\end{aligned}
$$

and

$$
\begin{aligned}
& \llbracket T_{\text {skew }}^{F / r}(t, \mathbf{q}) \rrbracket:=\int_{\Gamma_{2}(t, \mathbf{q})} \rho_{F}\left([N(t, \mathbf{q})]-[N(t, \mathbf{q})]^{T}\right) \otimes \varphi^{r} \mathrm{~d} \Gamma_{x} \\
& \quad+\frac{1}{2} \int_{\Gamma_{1}} \rho_{F}\left(\left[W^{r}\right]^{T} \mathbf{n} \otimes\left[D \varphi^{r}\right]\left[D \varphi^{r}\right]^{T}-\left[D \varphi^{r}\right]\left[D \varphi^{r}\right]^{T} \otimes\left[W^{r}\right]^{T} \mathbf{n}\right) \mathrm{d} \Gamma_{x} .
\end{aligned}
$$

The matrices $\left[M_{\text {sym }}^{F / d}(t, \mathbf{q})\right]$ and $\left[M_{\text {skew }}^{F / d}(t, \mathbf{q})\right]$ are given respectively by:

$$
\begin{aligned}
& \quad\left[M_{s y m}^{F / d}(t, \mathbf{q})\right]:=\int_{\Gamma_{2}(t, \mathbf{q})} \rho_{F}\left(\varphi^{r} \otimes \mathbf{A}+\mathbf{A} \otimes \varphi^{r}\right) \mathrm{d} \Gamma_{x} \\
& -\int_{\Gamma_{2}(t, \mathbf{q})} \rho_{F}\left(\mathbf{v}^{d} \cdot \mathbf{n}\right)\left[D \varphi^{r}\right]\left[D \varphi^{r}\right]^{T} \mathrm{~d} \Gamma_{x}+2 \int_{\Gamma_{2}(t, \mathbf{q})} \rho_{F}\left(\mathbf{v}^{d} \cdot \mathbf{n}\right)\left[W^{r}\right]^{T} \mathbf{n} \otimes\left[W^{r}\right]^{T} \mathbf{n} \mathrm{d} \Gamma_{x} \\
& -2 \int_{\Gamma_{2}(t, \mathbf{q})} \rho_{F} \mathcal{H}\left(\mathbf{v}^{d} \cdot \mathbf{n}\right)\left(\left[W^{r}\right]^{T} \mathbf{n} \otimes \varphi^{r}+\varphi^{r} \otimes\left[W^{r}\right]^{T} \mathbf{n}\right) \mathrm{d} \Gamma_{x}
\end{aligned}
$$

and

$$
\begin{aligned}
& {\left[M_{\text {skew }}^{F / d}(t, \mathbf{q})\right]:=\int_{\Gamma_{2}(t, \mathbf{q})} \rho_{F} \varphi^{d}\left([N]-[N]^{T}\right) \mathrm{d} \Gamma_{x}} \\
& \quad+\int_{\Gamma_{1}} \rho_{F}\left(\left[W^{r}\right]^{T} \mathbf{n} \otimes\left[D \varphi^{r}\right]\left(\nabla \varphi^{d}\right)^{T}-\left[D \varphi^{r}\right]\left(\nabla \varphi^{d}\right)^{T} \otimes\left[W^{r}\right]^{T} \mathbf{n}\right) \mathrm{d} \Gamma_{x}
\end{aligned}
$$

where $\kappa_{1}(t, \mathbf{q}, \mathbf{x})$ and $\kappa_{2}(t, \mathbf{q}, \mathbf{x})$ are the principal curvatures at the point $\mathbf{x}$ of $\Gamma_{2}(t, \mathbf{q})$ and the unitary vectors $\boldsymbol{\tau}_{1}(t, \mathbf{q}, \mathbf{x})$ and $\boldsymbol{\tau}_{2}(t, \mathbf{q}, \mathbf{x})$ are the associated principal directions such that $\left\{\mathbf{n}, \boldsymbol{\tau}_{1}, \boldsymbol{\tau}_{2}\right\}$ be a direct orthogonal basis and $[D \mathbf{n}] \boldsymbol{\tau}_{i}=-\kappa_{i} \boldsymbol{\tau}_{i}$, $i=1,2$. $\mathcal{H}(t, \mathbf{q}, \mathbf{x}):=\left(\kappa_{1}(t, \mathbf{q}, \mathbf{x})+\kappa_{2}(t, \mathbf{q}, \mathbf{x})\right) / 2$ is the mean curvature. Finally, 
the expressions of both vectors $\mathbf{F}^{F / d}(t, \mathbf{q})$ and $\mathbf{F}^{g}(t, \mathbf{q})$ are:

(D.5a)

$$
\begin{aligned}
\mathbf{F}^{F / d}(t, \mathbf{q}) & :=\int_{\Gamma_{2}(t, \mathbf{q})} \rho_{F}\left(\left(\dot{\mathbf{v}}^{d} \cdot \mathbf{n}\right) \varphi^{r}+\varphi^{d} \mathbf{A}\right) \mathrm{d} \Gamma_{x}+\frac{1}{2} \int_{\Gamma_{1}} \rho_{F}\left|\nabla \varphi^{d}\right|^{2}\left[W^{r}\right]^{T} \mathbf{n} \mathrm{d} \Gamma_{x} \\
+ & 2 \int_{\Gamma_{2}(t, \mathbf{q})} \rho_{F}\left(\mathbf{v}^{d} \cdot \mathbf{n}\right)^{2}\left[W^{r}\right]^{T} \mathbf{n} \mathrm{d} \Gamma_{x}-2 \int_{\Gamma_{2}(t, \mathbf{q})} \rho_{F} \mathcal{H}\left(\mathbf{v}^{d} \cdot \mathbf{n}\right) \varphi^{d}\left[W^{r}\right]^{T} \mathbf{n} \mathrm{d} \Gamma_{x} \\
- & \int_{\Gamma_{2}(t, \mathbf{q})} \rho_{F}\left(2 \mathcal{H}\left(\mathbf{v}^{d} \cdot \mathbf{n}\right)^{2}-\Pi_{2}\left(\mathbf{v}_{\Gamma}^{d}, \mathbf{v}_{\Gamma}^{d}\right)+2 \nabla_{\Gamma}\left(\mathbf{v}^{d} \cdot \mathbf{n}\right) \cdot \mathbf{v}^{d}\right) \varphi^{r} \mathrm{~d} \Gamma_{x} \\
& -\int_{\Gamma_{2}(t, \mathbf{q})} \rho_{F}\left(\mathbf{v}^{d} \cdot \mathbf{n}\right)\left[D \varphi^{r}\right]\left(\nabla \varphi^{d}\right)^{T} \mathrm{~d} \Gamma_{x},
\end{aligned}
$$

where $\Pi_{2}\left((t, \mathbf{q}, \mathbf{x}), \mathbf{v}_{\Gamma}^{d}, \mathbf{v}_{\Gamma}^{d}\right)=-\kappa_{1}\left(\mathbf{v}^{d} \cdot \boldsymbol{\tau}_{1}\right)^{2}-\kappa_{2}\left(\mathbf{v}^{d} \cdot \boldsymbol{\tau}_{2}\right)^{2}$ and

$$
\mathbf{F}^{b}(t, \mathbf{q}):=-\int_{S(t, \mathbf{q})}\left(\rho_{S}(\mathbf{x})-\rho_{F}\right) g\left[W^{r}\right]^{T} \mathbf{e}_{3} \mathrm{~d} \mathbf{x} .
$$

Observe that $F_{i}^{b}(t, \mathbf{q})=0$ for $i=4,5$ and, taking into account (2.6) and (2.7), $F_{6}^{b}(t, \mathbf{q})=\left(m-\rho_{F} \operatorname{vol}\left(S_{0}\right)\right) g$, where $\operatorname{vol}\left(S_{0}\right)$ is the volume of the swimmer and $g$ the gravitational constant.

\section{REFERENCES}

[1] C. Amrouche, V. Girault, and J. Giroire. Weighted Sobolev spaces for Laplace's equation in $\mathbf{R}^{n}$. J. Math. Pures Appl. (9), 73(6):579-606, 1994.

[2] C. Amrouche, V. Girault, and J. Giroire. Dirichlet and Neumann exterior problems for the $n$-dimensional Laplace operator: an approach in weighted Sobolev spaces. J. Math. Pures Appl. (9), 76(1):55-81, 1997.

[3] V. I. Arnold. Mathematical methods of classical mechanics. Springer-Verlag, New York, 1978. Translated from the Russian by K. Vogtmann and A. Weinstein, Graduate Texts in Mathematics, 60.

[4] G. Borelli. On the movement of animals. Springer-Verlag, New-York, 1989.

[5] T-. Z. Boulmezaoud. Espaces de Sobolev avec poids pour l'équation de Laplace dans le demiespace. C. R. Acad. Sci. Paris Sér. I Math., 328(3):221-226, 1999.

[6] C. M. Breder. The locomotion of fishes. Zoologica, 4:159-256, 1926.

[7] J. Carling, T. Williams, and G Bowtell. Self-propelled anguilliform swimming: simultaneous solution of the two-dimentional navier-stokes equations and newton's laws of motion. $J$. of Experimental Biology, 201:3143-3166, 1998.

[8] H. Cartan. Théorie élémentaire des fonctions analytiques d'une ou plusieurs variables complexes. Avec le concours de Reiji Takahashi. Enseignement des Sciences. Hermann, Paris, 1961.

[9] T. Chambrion and M. Sigalotti. Tracking control for an ellipsoidal submarine driven by kirchhoff's laws. Preprint in: Les prépublications de l'Institut Élie Cartan, $\mathrm{n}^{\circ} 35$, http://www.iecn.u-nancy.fr/Preprint, 2006.

[10] S. Childress. Mechanics of swimming and flying, volume 2 of Cambridge Studies in Mathematical Biology. Cambridge University Press, Cambridge, 1981.

[11] E-A. Coddington and N. Levinson. Theory of ordinary differential equations. McGraw-Hill Book Company, Inc., New York-Toronto-London, 1955.

[12] Houot J. G. and A. Munnier. On the motion and collisions of rigid bodies in an ideal fluid. Preprint in: Les prépublications de l'Institut Élie Cartan, $\mathrm{n}^{\circ} 33,2006$.

[13] G-. P. Galdi. On the steady self-propelled motion of a body in a viscous incompressible fluid. Arch. Ration. Mech. Anal., 148(1):53-88, 1999.

[14] G-. P. Galdi. On the motion of a rigid body in a viscous liquid: a mathematical analysis with applications. In Handbook of mathematical fluid dynamics, Vol. I, pages 653-791. NorthHolland, Amsterdam, 2002. 
[15] J. P. Giesing. Nonlinear two-dimensional unsteady potential flow with lift. J. of Aircraft, $5(2): 135-143,1968$.

[16] J. Gray. Study in animal locomotion iv-the propulsive powers of the dolphin. J. of experimental biology, 10:192-199, 1932.

[17] J. Gray. Study in animal locomotion i-the movement of a fish with special reference to the eel. J. of experimental biology, 13:88-104, 1936.

[18] A. Henrot and M. Pierre. Variation et optimisation de formes : une analyse géométrique, volume 048 of Mathématiques et applications. Springer, Berlin/Heidelberg/New York, 2005.

[19] T. I. Hesla. Collisions of smooth bodies in viscous fluids, a mathematical investigation. $\mathrm{PhD}$ thesis, University of Minnesota, May 2005.

[20] M. Hillairet. Lack of collision between solid bodies in a $2 \mathrm{~d}$ constant-density incompressible viscous flow. Submitted for publication.

[21] E. Kanso, J. E. Marsden, C. W. Rowley, and J. B. Melli-Huber. Locomotion of articulated bodies in a perfect fluid. J. Nonlinear Sci., 15(4):255-289, 2005.

[22] J. Katz and A. Plotkin. Low-speed aerodynamics: from wing theory to panel methods. McGraw-Hill, 1991.

[23] S. D. Kelly and R. M. Murray. Modelling efficient pisciform swimming for control. Internat. J. Robust Nonlinear Control, 10(4):217-241, 2000. Control of underactuated nonlinear systems.

[24] R. Krasny. A vortex-dipole sheet model for a wake. Phys. Fluids A, 1(2):173-175, 1989.

[25] H. Lamb. Hydrodynamics. Cambridge Mathematical Library. Cambridge University Press, Cambridge, sixth edition, 1993. With a foreword by R. A. Caflisch [Russel E. Caflisch].

[26] J. Lighthill. Mathematical biofluiddynamics. Society for Industrial and Applied Mathematics, Philadelphia, Pa., 1975. Based on the lecture course delivered to the Mathematical Biofluiddynamics Research Conference of the National Science Foundation held from July 16-20, 1973, at Rensselaer Polytechnic Institute, Troy, New York, Regional Conference Series in Applied Mathematics, No. 17.

[27] H. Liu and K. Kawachi. A numerical study of undulatory swimming. J. comput. phys., 155(2):223-247, 1999.

[28] R. Mason. Fluid Locomotion and Trajectory Planning for Shape-Changing Robots. Ph.d. thesis, California Institute of Technology, Department of Mechanical Engineering, June 2003.

[29] J. B. Melli, Rowley C. W., and D. S. Rufat. Motion planning for an articulated body in a perfect fluid. SIAM J. on Applied Dynamical Systems, 2006. To appear.

[30] M. Moubachir and J.-P. Zolésio. Moving shape analysis and control, volume 277 of Pure and Applied Mathematics (Boca Raton). Chapman \& Hall/CRC, Boca Raton, FL, 2006. Applications to fluid structure interactions.

[31] A. Munnier. On the self-displacement of deformable bodies in an ideal fluid. Preprint in: Les prépublications de l'Institut Élie Cartan, $n^{\circ} 46$, http://www.iecn.u-nancy.fr/Preprint, 2006.

[32] J. San Martin, J. F. Scheid, T. Takahashi, and M Tucsnak. An initial and boundary problem modeling fish-like swimming. Preprint in: Les prépublication de l'Institut Élie Cartan, $\mathrm{n}^{\circ} 27$, http://www.iecn.u-nancy.fr/Preprint, 2006.

[33] J. A. San Martín, V. Starovoitov, and M. Tucsnak. Global weak solutions for the twodimensional motion of several rigid bodies in an incompressible viscous fluid. Arch. Ration. Mech. Anal., 161(2):113-147, 2002.

[34] J. A. Sparenberg. Survey of the mathematical theory of fish locomotion. J. Engrg. Math., 44(4):395-448, 2002.

[35] G. Taylor. Analysis of the swimming of microscopic organisms. Proc. R. Soc. Lond., Ser. A, 209:447-461, 1951.

[36] G. Taylor. Analysis of the swimming of long and narrow animals. Proc. R. Soc. Lond., Ser. A, 214:158-183, 1952.

[37] M. S. Triantafyllou, G. S. Triantafyllou, and D. K. P. Yue. Hydrodynamics of fishlike swimming. In Annual review of fluid mechanics, Vol. 32, volume 32 of Annu. Rev. Fluid Mech., pages 33-53. Annual Reviews, Palo Alto, CA, 2000.

[38] J. L. Vázquez and E. Zuazua. Lack of collision in a simplified 1-dimensional model for fluidsolid interaction. Submitted for publication.

[39] T. Y. Wu. Mathematical biofluiddynamics and mechanophysiology of fish locomotion. Math. Methods Appl. Sci., 24(17-18):1541-1564, 2001. Biofluiddynamics. 
ON THE SELF-DISPLACEMENT OF DEFORMABLE BODIES IN A POTENTIAL FLUID FLOßB

[40] Q. Zhu, M. J. Wolfgang, D. K. P. Yue, and M. S. Triantafyllou. Three-dimensional flow structures and vorticity control in fish-like swimming. J. Fluid Mech., 468:1-28, 2002.

Insitut Elie Cartan UMR 7502, NAnCy-Université, CNRS, INRIA,, B.P. 239, F-54506 Vandoeuvre-lès-Nancy Cedex, France,, Alexandre.munnier@iecn.u-nancy.fr, And, INRIA Lorraine, Projet CORIDA. 